TRANSACTIONS OF THE

AMERICAN MATHEMATICAL SOCIETY

Volume 357, Number 12, Pages 4957-5000

S 0002-9947(05)03644-5

Article electronically published on March 10, 2005

\title{
REGULATING FLOWS, TOPOLOGY OF FOLIATIONS AND RIGIDITY
}

\author{
SÉRGIO R. FENLEY
}

\begin{abstract}
A flow transverse to a foliation is regulating if, in the universal cover, an arbitrary orbit of the flow intersects every leaf of the lifted foliation. This implies that the foliation is $\mathbf{R}$-covered, that is, its leaf space in the universal cover is homeomorphic to the reals. We analyse the converse of this implication to study the topology of the leaf space of certain foliations. We prove that if a pseudo-Anosov flow is transverse to an $\mathbf{R}$-covered foliation and the flow is not an $\mathbf{R}$-covered Anosov flow, then the flow is regulating for the foliation. Using this we show that several interesting classes of foliations are not $\mathbf{R}$-covered. Finally we show a rigidity result: if an $\mathbf{R}$-covered Anosov flow is transverse to a foliation but is not regulating, then the foliation blows down to one topologically conjugate to the stable or unstable foliations of the transverse flow.
\end{abstract}

\section{INTRODUCTION}

In this article we study the topology of foliations in 3-manifolds as influenced by a transverse pseudo-Anosov flow. A Reebless foliation in a closed 3-manifold lifts to a foliation in the universal cover. It is very natural to ask what is the topology of the leaf space in the universal cover. The basic form of this is to check whether the leaf space is Hausdorff or not. The Reebless condition implies that this leaf space is always a one-dimensional manifold $[\mathrm{No}$. Consequently if it is Hausdorff it will be homeomorphic to the real numbers $\mathbf{R}$. In this case we call the foliation an $\mathbf{R}$-covered foliation [Fe3, Pl4]. An immediate consequence of being $\mathbf{R}$-covered is that the fundamental group acts on $\mathbf{R}$, and this action can be very useful [Pl3]. One important question addressed here is: when is a foliation $\mathbf{R}$-covered?

It turns out that in general it seems to be hard and subtle to check whether a foliation is $\mathbf{R}$-covered or not. Fibrations over the circle are always $\mathbf{R}$-covered and finite depth foliations Ga1, Ga2 are never $\mathbf{R}$-covered unless the compact leaves are fibers of fibrations over the circle [Fe1].

For stable or unstable foliations of Anosov flows the question is much more complex and interesting. There is the groundbreaking work of Verjovsky [Ve, who was the first to analyse the topology of the lifts of the stable/unstable foliations of an Anosov flow and its important consequences. Suspension Anosov flows (suspensions) and geodesic flows in the unit tangent bundle of closed surfaces of negative curvature (geodesic flows) have stable/unstable foliations which are $\mathbf{R}$-covered.

Received by the editors January 3, 2002 and, in revised form, February 1, 2004.

2000 Mathematics Subject Classification. Primary 37D20, 53C12, 53C23, 57R30; Secondary 37C85, 57M99.

This research was partially supported by NSF grants DMS-9612317 and DMS-0071683. 
Anosov flows in Seifert fibered spaces are completely understood, due to the work of Ghys Gh. He proved that the stable foliation of the flow is always $\mathbf{R}$-covered and this was an essential step to show that such flows are always topologically conjugate to geodesic flows. Anosov flows in graph manifolds are also well understood due to the work of Barbot [Ba1, Ba3, Ba4] and there are many $\mathbf{R}$-covered examples. Again the $\mathbf{R}$-covered property for the stable foliation had important consequences. Also there are many non-R-covered examples in graph manifolds. In hyperbolic manifolds there are many examples of $\mathbf{R}$-covered Anosov foliations obtained by Dehn surgery on closed orbits of suspensions or geodesic flows [Fe3]. This has strong consequences for the geometry of the flows in hyperbolic manifolds, namely the study of the quasigeodesic property for the flow lines - when the stable foliation is $\mathbf{R}$-covered, then the flow is not a quasigeodesic flow [Fe3]. In both graph manifolds and hyperbolic manifolds, the proof of the $\mathbf{R}$-covered property involves a very tricky analysis - for hyperbolic manifolds this entailed the study of how Dehn surgery modified the topology of the leaf space in the universal cover [Fe3]. The results were obtained under very controlled circumstances and in general Dehn surgery on suspensions may produce non-R-covered Anosov foliations; see [Fe5] for non-R-covered examples where the surgered manifold is hyperbolic.

Another class of $\mathbf{R}$-covered foliations is that of "slitherings". A slithering of a manifold over the circle is a fibration of the universal cover of the manifold over the circle which is equivariant under group translations and produces a foliation in the manifold Th5]. By construction these are all R-covered. Fibrations over the circle are examples of slitherings, as are stable foliations of a large class of Anosov flows [Th5, Fe3]. Furthermore there are several techniques to construct or replicate slitherings, for instance branched covering techinques [Th5, Ca1]. This makes slitherings very common.

Finally there are also some new constructions by Calegari [Ca1] of $\mathbf{R}$-covered foliations in hyperbolic 3-manifolds which are not associated to slitherings. Another important fact is that for a general foliation in a closed, hyperbolic 3-manifold, the $\mathbf{R}$-covered property implies that every leaf in the universal cover has limit set which is the entire sphere at infinity $\mathrm{Fe} 7$.

On the other hand, the failure of the $\mathbf{R}$-covered property for the stable/unstable foliations of an Anosov flow in a 3-manifold also has strong consequences for the dynamics of the flow and the topology of the manifold [Fe4, Fe5]. In this case the general structure of such leaf spaces is not yet well understood.

All of these examples demonstrate the importance of knowing whether a foliation is $\mathbf{R}$-covered or not. When $\mathcal{F}$ has a compact leaf $B$, then $\mathcal{F}$ is $\mathbf{R}$-covered if and only if $B$ is a virtual fiber of $M$ over the circle Go-Sh. However when $\mathcal{F}$ has no compact leaves, it is very difficult to analyse the $\mathbf{R}$-covered property and very little is known beyond the examples mentioned above.

In this article we analyse this question when there is a transverse pseudo-Anosov flow. First we mention various classes of examples where there is a pseudo-Anosov flow transverse to the foliation. 1) If the manifold fibers over the circle with pseudoAnosov monodromy, then there is a pseudo-Anosov flow transverse to the fibration Th3, Bl-Ca. This flow gives substantial information about the manifold and the foliation Th2, Th4, Ca-Th. 2) Another example is the situation of finite depth foliations: there are compact leaves, all leaves are properly embedded and the foliation strongly reflects a topological hierarchy of the manifold [Ga1, Ga2]. Gabai 
showed that if the manifold is orientable, irreducible and has non-trivial second homology, then it has Reebless finite depth foliations Ga1, Ga2. Work of Gabai and Mosher [Mo2] shows that, as in the fibering case, there is a pseudo-Anosov flow transverse to the foliation (in general the flow is "almost" transverse to the foliation - a technical condition). The stable/unstable laminations obtained by splitting the singular leaves of the stable/unstable singular foliations of the flow are used to prove important results on most Dehn surgeries on knot complements in $\mathbf{S}^{3}$ Mo2 . 3) Work of Roberts Rob1, Rob2] implies that if $\gamma$ is a knot in $\mathbf{S}^{3}$ with hyperbolic complement which fibers over the circle, then there are infinitely many Dehn surgeries on $\gamma$ yielding closed manifolds with foliations transverse to pseudo-Anosov flows. 4) About two years ago, Thurston showed that a foliation induced by a slithering of the manifold over the circle is either reducible or admits a transverse pseudo-Anosov flow [Th5]. 5) We recently extended this result to general $\mathbf{R}$-covered foliations:

Theorem $([\mathrm{Fe} 10])$. Let $\mathcal{F}$ be a transversely oriented, $\mathbf{R}$-covered foliation in $M^{3}$ closed, aspherical. Then either there is a $\mathbf{Z} \oplus \mathbf{Z}$ subgroup of $\pi_{1}(M)$ or there is a pseudo-Anosov flow transverse to $\mathcal{F}$. In particular the second option occurs if $M$ is hyperbolic.

This result was independently proved by Calegari Ca2. 6) Finally Calegari has shown the same result for foliations with one-sided branching [Ca3] and minimal foliations Ca4.

Pseudo-Anosov flows transverse to foliations are amenable to branched cover and cut-and-paste techniques. This will be explored in this article to analyse two classes of examples. See also the wealth of examples created by Cooper, Long and Reid [CLR1, CLR2] and the author [Fe8] for compact immersed surfaces transverse to pseudo-Anosov flows. Thurston proposed that the existence of a pseudo-Anosov flow transverse to a foliation is a very general situation Th6, Th7.

Then let $\Phi$ be a pseudo-Anosov transverse to a foliation $\mathcal{F}$. The question considered here is: can the transverse pseudo-Anosov flow be used to decide whether $\mathcal{F}$ is $\mathbf{R}$-covered or not? First consider the following: does an arbitrary flow line in the universal cover intersect every leaf of the lifted foliation? If this happens we say that $\Phi$ regulates $\mathcal{F}$ Th5, or that $\Phi$ is a regulating flow. Clearly if this is the case, then the foliation is $\mathbf{R}$-covered - points in the given flow line parametrize the leaf space as homeomorphic to $\mathbf{R}$. Our goal is to analyse the converse: does $\mathcal{F}$ being $\mathbf{R}$-covered imply that $\Phi$ is regulating for $\mathcal{F}$ ? Surprisingly this is not true in general and there are easy counterexamples. However we prove that the converse only fails for some very restricted and well-understood situations.

Let $\mathcal{F}^{s}, \mathcal{F}^{u}$ be the singular stable/unstable foliations associated to the pseudoAnosov flow $\Phi$. A pseudo-Anosov flow is called $\mathbf{R}$-covered if one of its stable or unstable foliations is $\mathbf{R}$-covered Ba1, Fe3. If a pseudo-Anosov flow is $\mathbf{R}$-covered, then there are no $p$-prong orbits and also both foliations $\mathcal{F}^{s}, \mathcal{F}^{u}$ are $\mathbf{R}$-covered Ba1, Fe3. In particular this implies that $\Phi$ is non-singular and is therefore an Anosov flow. Hence we will usually refer to those flows as R-covered Anosov flows.

Given a pseudo-Anosov flow $\Phi$ transverse to a foliation $\mathcal{F}$ in $M$, let $\widetilde{\Phi}$ be the lifted flow to the universal cover $\widetilde{M}$ and let $\widetilde{\mathcal{F}}$ be the lifted foliation. We first study basic properties of foliations transverse to pseudo-Anosov flows. 
Theorem A. Let $\Phi$ be a pseudo-Anosov flow transverse to a foliation $\mathcal{F}$ in $M^{3}$ closed. Then $\mathcal{F}$ is Reebless. In $\widetilde{M}$, a given orbit of $\widetilde{\Phi}$ intersects a leaf of $\widetilde{\mathcal{F}}$ at most once.

It does not follow that $\mathcal{F}$ is taut Ga1]; see constructions in section 3. Then we prove the main result:

Main Theorem. Let $\Phi$ be a pseudo-Anosov flow transverse to a foliation $\mathcal{F}$ in $M^{3}$ closed. Suppose that $\Phi$ is not an $\mathbf{R}$-covered Anosov flow. Then $\Phi$ is regulating if and only if $\mathcal{F}$ is an $\mathbf{R}$-covered foliation. There are counterexamples when $\Phi$ is an $\mathbf{R}$-covered pseudo-Anosov flow.

The rough idea is as follows: as $\mathcal{F}$ is $\mathbf{R}$-covered, there is a unique way to move transversally to $\widetilde{\mathcal{F}}$ in $\widetilde{M}$. When $\Phi$ is not regulating for $\mathcal{F}$, this unique transversal direction to $\widetilde{\mathcal{F}}$ will force the creation of a unique transversal direction to say the stable foliation $\widetilde{\mathcal{F}}^{s}$ of $\widetilde{\Phi}$. This in turn implies that the leaf space of $\widetilde{\mathcal{F}}^{s}$ is $\mathbf{R}$ and hence $\Phi$ is an $\mathbf{R}$-covered Anosov flow. The fundamental tool used here is the topological theory of pseudo-Anosov flows as developed in [Fe5, Fe9].

When $\Phi$ is an $\mathbf{R}$-covered Anosov flow, counterexamples to the main theorem can be obtained as follows: let $\mathcal{F}$ be the stable foliation of $\Phi$ - which is $\mathbf{R}$-covered. Perturb $\Phi$ to nearby $\Phi^{\prime}$ which is transverse to $\mathcal{F}$. Then $\Phi^{\prime}$ will not be regulating for $\mathcal{F}$. This is explained in detail in section 5 .

The main theorem is a useful tool to analyse the topology of foliations:

Theorem B. There is an infinite family of examples of non-R-covered foliations obtained by branched covers of foliations transverse to suspension Anosov flows and to skewed $\mathbf{R}$-covered Anosov flows. The resulting foliations are transverse to pseudo-Anosov flows. There are also infinitely many examples obtained by cut-andpaste operations on fibrations over the circle.

The skewed condition is explained in detail in section 5 .

The main theorem leaves open the situation where $\Phi$ is an $\mathbf{R}$-covered Anosov flow. In this case we prove a strong topological rigidity. We do this by characterizing all the possible foliations transverse to such a flow. A foliation $\mathcal{F}_{1}$ is a blow up of another foliation $\mathcal{F}_{2}$ if $\mathcal{F}_{1}$ is obtained by blowing up a collection of leaves of $\mathcal{F}_{2}$ into foliated $I$-bundles. We prove:

Theorem $\mathbf{C}$ (rigidity theorem). Let $\Phi$ be an $\mathbf{R}$-covered Anosov flow transverse to a foliation $\mathcal{F}$. Then $\mathcal{F}$ is $\mathbf{R}$-covered. In addition if $\Phi$ is not regulating for $\mathcal{F}$, then $\mathcal{F}$ is topologically conjugate to a blow up of either the stable or unstable foliation of $\Phi$.

This topological rigidity result says that the only counterexamples to the main theorem are essentially obtained by the construction mentioned above and detailed in section 5. The proof of this rigidity result takes about a third of the article and is left to the end. As is usual with rigidity results it uses tools from a variety of sources. Here we need: the structure of $\mathbf{R}$-covered Anosov foliations, topology of foliations, Gromov negatively curved spaces, quasi-isometries of metric spaces, dynamics and topological conjugacy of flows, amongst others. The proof is achieved by producing a new flow which is topologically conjugate to the original flow and which is tangent to a blow down of the foliation $\mathcal{F}$. 
The results of this article have several applications. In a subsequent article we analyse the atoroidal property for manifolds with pseudo-Anosov flows and prove:

Theorem ([Fe11] $)$. Let $\Phi$ be a pseudo-Anosov flow in $M^{3}$ closed. Let $\mathbf{A}=\mathbf{Z} \oplus \mathbf{Z}$ be a subgroup of the fundamental group $\pi_{1}(M)$. Then $\mathbf{A}$ does not act freely in the orbit space of $\widetilde{\Phi}$, unless $\Phi$ is topologically conjugate to a suspension Anosov flow. This means that some non-trivial element of $\mathbf{A}$ leaves invariant an orbit of $\widetilde{\Phi}$. Hence if $\Phi$ is not topologically conjugate to a suspension Anosov flow, then $\mathbf{A}$ represents a free homotopy from a closed orbit of $\Phi$ to itself.

Together with the results here one obtains an important relationship with the topology of the underlying manifold:

Theorem ([Fe11]). Let $\Phi$ be a pseudo-Anosov flow transverse to an $\mathbf{R}$-covered foliation $\mathcal{F}$ in $M^{3}$ closed. Then $M$ is atoroidal, unless $\Phi$ is a non-regulating $\mathbf{R}$ covered Anosov flow or $\Phi$ is topologically conjugate to a suspension Anosov flow. The condition is necessary as simple examples show.

We can also apply the results here to obtain topological rigidity of pseudo-Anosov flows transverse to foliations. This means characterizing all pseudo-Anosov flows transverse to a given foliation.

Theorem ([Fe12] $)$. Let $\Phi$ be a regulating pseudo-Anosov flow transverse to an $\mathbf{R}$ covered foliation $\mathcal{F}$ in closed $M^{3}$. Then up to topological conjugacy, $\Phi$ is the only regulating pseudo-Anosov flow transverse to $\mathcal{F}$.

The article is organized as follows: the next section contains general results about foliations transverse to pseudo-Anosov flows. Section 3 contains a review of the topological theory of pseudo-Anosov flows as needed here. In section 4 we prove the main theorem and in the next section we produce the counterexamples in the case of $\mathbf{R}$-covered Anosov flows. In section 6 we use the main theorem to study the topology of some classes of foliations and prove theorem B. In the last section we show that the counterexamples to the main theorem can only occur in a very restricted setting and prove theorem $\mathrm{C}$.

We thank Bill Thurston for enlightening and very enjoyable conversations about foliations and flows. The main result in this article was motivated by a question asked by him. He also suggested that we analyse the branched cover examples of section 6 .

\section{Foliations transverse to PSEUdo-Anosov Flows}

Pseudo-Anosov flows are a generalization of suspension flows of pseudo-Anosov surface homeomorphisms. These flows behave much like Anosov flows, but they may have finitely many singular orbits which are periodic and have a prescribed behavior.

Let $\Phi$ be a flow on a closed, oriented 3-manifold $M$. We say that $\Phi$ is a pseudoAnosov flow if the following are satisfied:

- For each $x \in M$, the flow line $t \rightarrow \Phi(x, t)$ is $C^{1}$, it is not a single point, and the tangent vector bundle $D_{t} \Phi$ is $C^{0}$.

- There is a finite number of periodic orbits $\left\{\gamma_{i}\right\}$, called singular orbits, such that the flow is "topologically" smooth off of the singular orbits (see below).

- The flowlines are tangent to two singular transverse foliations $\mathcal{F}^{s}, \mathcal{F}^{u}$ which have smooth leaves off of $\gamma_{i}$ and intersect exactly in the flow lines of $\Phi$. These are 

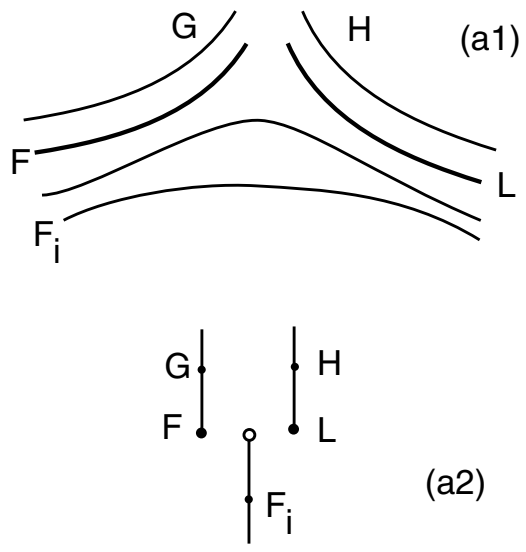

(a2)

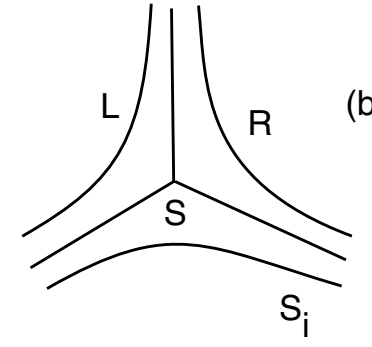

(b1)

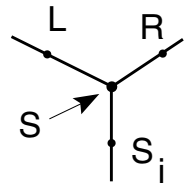

Figure 1. (a) Non-Hausdorff behavior in the leaf space of $\widetilde{\mathcal{F}}^{s}$ : (a1) $F, L$ non-separated from each other, as seen in $\widetilde{M}$, (a2) the corresponding picture in the leaf space $\mathcal{H}^{s}$. (b) A singular leaf of $\widetilde{\mathcal{F}}^{s}$ : (b1) as seen in $\widetilde{M},(\mathrm{~b} 2)$ as seen in $\mathcal{H}^{s}$.

like Anosov foliations off of the singular orbits. This is the topologically smooth behavior described above. A leaf containing a singularity is homeomorphic to $P \times$ $I / f$, where $P$ is a $p$-prong in the plane and $f$ is a homeomorphism from $P \times\{1\}$ to $P \times\{0\}$. We restrict to $p$ at least 2, that is, we do not allow 1-prongs.

- In a stable leaf all orbits are forward asymptotic, in an unstable leaf all orbits are backwards asymptotic.

Notation/definition: The singular foliations lifted to $\widetilde{M}$ are denoted by $\widetilde{\mathcal{F}}^{s}, \widetilde{\mathcal{F}}^{u}$. If

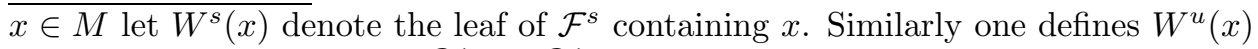
and in the universal cover $\widetilde{W}^{s}(x), \widetilde{W}^{u}(x)$. Similarly if $\alpha$ is an orbit of $\Phi$ define $W^{s}(\alpha)$, etc. Also let $\widetilde{\Phi}$ be the lifted flow to $\widetilde{M}$.

In Figure 1 we highlight the difference between non-Hausdorff behavior in the leaf space of $\widetilde{\mathcal{F}}^{s}$ and the splitting (or branching) of leaves associated to singular orbits of $\widetilde{\Phi}$. In part (a) the leaves $F, L$ of $\widetilde{\mathcal{F}}^{s}$ are not separated from each other in the leaf space of $\widetilde{\mathcal{F}}^{s}$. Notice that the sequence $F_{i}$ converges to $F$ and $L$. In Figure 1 part (b) we sketch a singular leaf $S$ with 3 prongs. Even though $S$ separates $\widetilde{M}$ into 3 or more regions, non Hausdorffness is not involved. The leaves $S_{i}$ converge only to $S$. In this article, unless otherwise specified, all pictures of leaves of $\widetilde{\mathcal{F}}^{s}, \widetilde{\mathcal{F}}^{u}$ will describe them as subsets of $\widetilde{M}$, rather than in the leaf space of $\widetilde{\mathcal{F}}^{s}$.

Let $\Phi$ be a pseudo-Anosov flow in a closed 3-manifold $M$. The following facts and definitions will be needed. We proved in Fe-Mo that the orbit space of $\widetilde{\Phi}$ in $\widetilde{M}$ is homeomorphic to the plane $\mathbf{R}^{2}$. This orbit space is denoted by $\mathcal{O} \cong \widetilde{M} / \widetilde{\Phi}$. Let $\Theta: \widetilde{M} \rightarrow \mathcal{O} \cong \mathbf{R}^{2}$ be the projection map. For any subset $B \subset \mathcal{O}$, let $B \times \mathbf{R} \subset \widetilde{M}$, be the set $\Theta^{-1}(B)$. As the foliations $\widetilde{\mathcal{F}}^{s}, \widetilde{\mathcal{F}}^{u}$ are invariant under $\widetilde{\Phi}$, they induce singular, transverse 1-dim foliations $\widetilde{\mathcal{F}}_{\mathcal{O}}^{s}, \widetilde{\mathcal{F}}_{\mathcal{O}}^{u}$ in $\mathcal{O}$. The singular points of $\widetilde{\mathcal{F}}_{\mathcal{O}}^{u}$ are the same as those of $\widetilde{\mathcal{F}}_{\mathcal{O}}^{s}$. If $L$ is a leaf of $\widetilde{\mathcal{F}}^{s}$ or $\widetilde{\mathcal{F}}^{u}$, then $\Theta(L) \subset \mathcal{O}$ is a tree which 
is either homeomorphic to $\mathbf{R}$ if $L$ is regular, or is a union of $p$-rays all with the same starting point if $L$ has a singular $p$-prong orbit. In particular every orbit in $L$ disconnects $L$.

In this section we develop some preliminaries of foliations transverse to pseudoAnosov flows. Let $\mathcal{F}$ be a foliation transverse to a pseudo-Anosov flow $\Phi$. Then $\mathcal{F}, \mathcal{F}^{s}, \mathcal{F}^{u}$ are 3 foliations in $M$ which are transverse to each other everywhere.

We now prove theorem A of the introduction.

Theorem 2.1 (Theorem A). Let $\Phi$ be a pseudo-Anosov flow transverse to a foliation $\mathcal{F}$ in $M^{3}$. Then $\mathcal{F}$ is Reebless. In addition every orbit of $\widetilde{\Phi}$ intersects a leaf of $\widetilde{\mathcal{F}}$ at most once.

Proof. Suppose that $\mathcal{F}$ is not Reebless and first consider a Reeb component which is a solid torus $V$ bounded by a torus $T$. Assume the flow is incoming along $T$. Since orbits are trapped inside once they enter $V$, the shadow lemma for pseudoAnosov flows [Han, Man, Mo1 shows that there is a periodic orbit $\gamma$ in $V-T$. Then $W^{u}(\gamma)$ is contained in $V-T$, because the flow is incoming along $T$. Since $\gamma^{n}$ is not null homotopic for any $n \neq 0$ [Mo1, then $\widetilde{V}$ is homeomorphic to $D^{2} \times \mathbf{R}$. It now follows that $\widetilde{W}^{u}(\gamma)$ is not properly embedded in the universal cover, a contradiction Ga-Oe. The same proof shows that there cannot be non-orientable Reeb components - that is the quotient of a Reeb component by an orientation reversing involution of $V$ which preserves the meridian. This can be seen by lifting to an orientation double cover of $M$ : the lifted flow is pseudo-Anosov transverse to a Reeb component.

As for the second statement: Since $\mathcal{F}$ is Reebless and transverse to a pseudoAnosov flow, then leaves of $\widetilde{\mathcal{F}}$ are properly embedded topological planes in $\widetilde{M}$, because closed transversals to $\mathcal{F}$ are not null homotopic [No. Therefore leaves of $\widetilde{\mathcal{F}}$ separate $\widetilde{M}$. Since orbits of $\widetilde{\Phi}$ are transverse to $\widetilde{\mathcal{F}}$, an orbit of $\widetilde{\Phi}$ can intersect a leaf of $\widetilde{\mathcal{F}}$ at most once.

Suppose that $\Phi$ is not regulating. Let $\Theta: \widetilde{M} \rightarrow \mathcal{O}$ be the projection. There is an orbit $\gamma$ of $\widetilde{\Phi}$ not intersecting all leaves of $\widetilde{\mathcal{F}}$ and a leaf $F$ of $\widetilde{\mathcal{F}}$ not intersecting all orbits of $\widetilde{\Phi}$. Hence $\Theta(F)$ is not all of $\mathcal{O}$. The following result describes the boundary of $\Theta(F) \subset \mathcal{O}$.

If $L \in \widetilde{\mathcal{F}}^{s}$, then a sector of $L$ is a component of $\widetilde{M}-L$. A line leaf of $L$ is the boundary $L^{\prime}$ of a sector of $L$. The line leaf $L^{\prime}$ is regular on the side which is a sector of $L$. It is regular on both sides if and only if $L$ is a regular leaf. The same terminology (sector, line leaf, etc.) is used for the corresponding objects of $\widetilde{\mathcal{F}}_{\mathcal{O}}^{s}, \widetilde{\mathcal{F}}_{\mathcal{O}}^{u}$ in $\mathcal{O}$.

Proposition 2.2. $\partial \Theta(F) \subset \mathcal{O}$ is a disjoint union of line leaves of $\widetilde{\mathcal{F}}_{\mathcal{O}}^{s}, \widetilde{\mathcal{F}}_{\mathcal{O}}^{u}$, all regular on the side containing $\Theta(F)$. Also if $L \in \widetilde{\mathcal{F}}^{s}$ (or $\widetilde{\mathcal{F}}^{u}$ ), then $F \cap L$ is connected.

Proof. Because $\Phi$ is transverse to $\mathcal{F}$ and $M$ is compact, then for $\epsilon>0$ sufficiently small there is $\eta(\epsilon)$ with $\eta(\epsilon) \rightarrow 0$, when $\epsilon \rightarrow 0$ so that any orbit of $\widetilde{\Phi}$ which comes within $\epsilon$ of a point $z \in F \in \widetilde{\mathcal{F}}$ will in fact intersect $F$ within $\eta(\epsilon)$ of $z$. Let $p \in \partial \Theta(F)$ and let $p_{i} \in \Theta(F)$ with $p_{i} \rightarrow p$. Let $z \in \widetilde{M}$ with $\Theta(z)=p$. Consider $D \subset \widetilde{M}$ a small embedded disk, transverse to $\widetilde{\Phi}$ with $z \in \operatorname{int}(D)$ and $\Theta$ injective in $D$. Let 


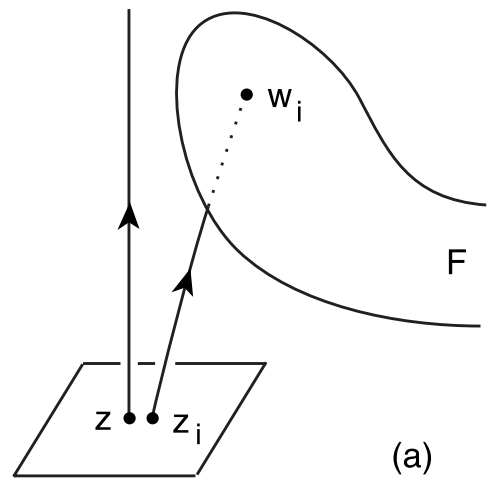

Figure 2. (a) Producing boundary in $\Theta(F)$. (b) Walls in the back of $F$.

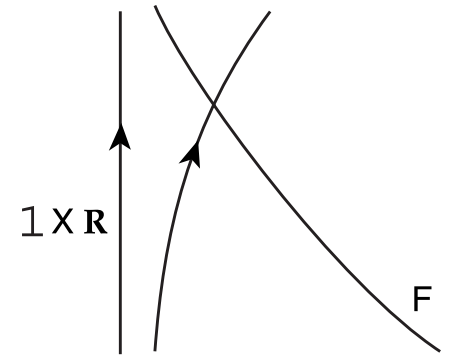

(b)

$w_{i} \in F$ with $\Theta\left(w_{i}\right)=p_{i}$. By truncating finitely many terms if necessary, there are unique $z_{i} \in D$ and $t_{i} \in \mathbf{R}$ so that $w_{i}=\widetilde{\Phi}_{t_{i}}\left(z_{i}\right)$. If $\left|t_{i}\right| \nrightarrow+\infty$ assume up to subsequence that $t_{i} \rightarrow t_{0}$. It follows that $w_{i} \rightarrow \widetilde{\Phi}_{t_{0}}(z)$. But since $F$ is closed in $\widetilde{M}$, then $\widetilde{\Phi}_{t_{0}}(z) \in F$, contradicting $p \notin \Theta(F)$.

Assume then that there is a subsequence $t_{i} \rightarrow+\infty$. Suppose that the corresponding $p_{i}$ are all in the closure of the same sector defined by $m=\Theta\left(\widetilde{W}^{s}(z)\right)$ at $p$. Let $l$ be the line leaf of $m$ which bounds this sector.

$\underline{\text { Claim }-~} l$ is a boundary leaf of the projection of $F$, that is, $l \subset \partial \Theta(F)$; see Figure 2(b).

Let $v \in \widetilde{M}$ with $\Theta(v) \in l$. Choose $\beta$ a small segment in $\widetilde{W}^{u}(v)$ transverse to the flow $\widetilde{\Phi}$ in $\widetilde{W}^{u}(v)$ and with $v$ in the interior of $\beta$. For $i$ big enough let $q_{i}=\beta \cap \widetilde{W}^{s}\left(w_{i}\right)$. The intersection is non-void because $l$ is regular on the side containing $p_{i}$. There are

$$
s_{i} \in \mathbf{R}, \quad s_{i} \rightarrow+\infty \text { so that } \widetilde{\Phi}_{s_{i}}\left(q_{i}\right) \in \widetilde{W}^{s}\left(w_{i}\right) \text { and } d\left(\widetilde{\Phi}_{s_{i}}\left(q_{i}\right), w_{i}\right) \rightarrow 0 .
$$

This implies that there are $\epsilon_{i} \rightarrow 0$ with $\widetilde{\Phi}_{s_{i}+\epsilon_{i}}\left(q_{i}\right) \in F$. Hence for $i$ big enough $\Theta\left(q_{i}\right) \in \Theta(F)$. In fact this shows that a segment $\alpha$ in $\widetilde{W}^{s}\left(q_{i}\right)$ transverse to $\widetilde{\Phi}$ in $\widetilde{W}^{s}\left(q_{i}\right)$ and going from $\widetilde{\Phi}_{s_{i}}\left(q_{i}\right)$ to $w_{i}$ projects into $\Theta(F)$. One concludes that

$$
\Theta(v) \in \Theta(F) \cup \partial \Theta(F) .
$$

If $\Theta(v) \in \Theta(F)$ let $E$ be a small disk contained in $F$ with $v$ in the interior of $\Theta(E)$. Hence for $i$ big enough there are bounded $r_{i}$ with $\widetilde{\Phi}_{r_{i}}\left(q_{i}\right) \in E$. But the argument above shows that there are $\widetilde{\Phi}_{s_{i}+\epsilon_{i}}\left(q_{i}\right) \in F$ with $s_{i}+\epsilon_{i} \rightarrow+\infty$ as $i \rightarrow+\infty$. This would produce two points $\widetilde{\Phi}_{r_{i}}\left(q_{i}\right)$ and $\widetilde{\Phi}_{s_{i}+\epsilon_{i}}\left(q_{i}\right)$ of $F$ in $\widetilde{\Phi}_{\mathbf{R}}\left(q_{i}\right)$, a contradiction.

We conclude that the stable line leaf $l \subset \partial \Theta(F)$, showing the claim.

Similarly given the $t_{i}$ defined above, if there is some subsequence $t_{i_{n}} \rightarrow-\infty$, then we have an unstable line leaf $I$ through $p$ with $I \subset \partial \Theta(F)$. Since $\Theta(F)$ is connected this would force $I \cap \Theta(F) \neq \emptyset$ which is impossible. In addition $l$ is regular on the side containing $F$. This proves the first statement. 
Now let $F \in \widetilde{\mathcal{F}}$ and $L \in \widetilde{\mathcal{F}}^{s}$. Suppose that $F \cap L$ has two components $u_{1}, u_{2}$. Then $\Theta\left(u_{1}\right) \cap \Theta\left(u_{2}\right)=\emptyset$ and there is $x \in L$ with $\Theta(x)$ separating $\Theta\left(u_{1}\right)$ from $\Theta\left(u_{2}\right)$ in $\Theta(L)$ and $\Theta(x) \in \partial \Theta\left(u_{1}\right)$. By the proof above $\Theta(x) \in \partial \Theta(F)$ and there is a line leaf

$$
m \subset \partial \Theta(F) \text { with either } m \subset \Theta\left(\widetilde{W}^{s}(x)\right) \text { or } m \subset \Theta\left(\widetilde{W}^{u}(x)\right) \text {. }
$$

Since $\Theta\left(u_{1}\right) \subset \Theta(F), \Theta\left(u_{1}\right) \subset \Theta\left(\widetilde{W}^{s}(x)\right)$ and $m$ is regular on the side containing $\Theta(F)$, then $m \subset \Theta\left(\widetilde{W}^{s}(x)\right)$ cannot happen. Now $\Theta\left(u_{1}\right)$ and $\Theta\left(u_{2}\right)$ must be in the same component of $\mathcal{O}-m$ and $\Theta(x)$ separates $\Theta\left(u_{1}\right)$ from $\Theta\left(u_{2}\right)$ in $\Theta(l)$. Hence there is a component of $\Theta\left(\widetilde{W}^{u}(x)\right)-\Theta(x)$ contained in $\Theta(F)$ and separating $\Theta\left(u_{1}\right)$ from $\Theta\left(u_{2}\right)$ in $\Theta\left(\widetilde{W}^{s}(x)\right)$. This contradicts the fact that $m$ is a line leaf of $\Theta\left(\widetilde{W}^{u}(x)\right)$ which is regular on the side containing $\Theta(F)$. The second statement follows. This finishes the proof of proposition 2.2 .

Notice that $\mathcal{F}$ is always transversely orientable because it is transverse to a nonvanishing continuous vector field. Let positive flow direction define the positive transversal orientation to $\mathcal{F}$. This will be fixed throughout the article.

As leaves of $\widetilde{\mathcal{F}}$ separate $\widetilde{M}$, then given $F \in \widetilde{\mathcal{F}}$ we can define the positive or front side of $F$ to be the component of $\widetilde{M}-F$ on the positive side of $F$ with respect to the transversal orientation to $\widetilde{\mathcal{F}}$. Similarly define the negative or back side of $F$. Notice that if $l$ is a stable boundary leaf of $\Theta(F)$, then $l \times \mathbf{R}$ is in the negative side of $F$; see Figure 2(b). In addition for any $z \in \widetilde{M}$ with $\Theta(z) \in l$ then $\widetilde{W}^{u}(z)$ intersects $F$. Similarly if $l$ is an unstable boundary leaf of $\partial \Theta(F)$, then $l \times \mathbf{R}$ is on the positive side of $F$. These facts will be used throughout the article.

We stress that the fact that $\mathcal{F}$ is transverse to a pseudo-Anosov flow does not imply that $\mathcal{F}$ is taut, where taut means that every leaf intersects a closed, tranverse loop Ga1. For example this can be seen with the Franks-Williams example of an intransitive Anosov flow Fr-Wi]. Start with a suspension Anosov flow $\Phi_{0}$ in $M_{0}$ and do a DA operation on a closed orbit $\gamma$ of $\Phi_{0}$. This produces a flow called derived from Anosov flow where $\gamma$ is now a repelling orbit. Remove a solid torus neighborhood of $\gamma$ obtaining a manifold $M_{1}$ with torus boundary $T$ and an incoming semi-flow $\Phi_{1}$. Consider a homeomorphic copy $M_{2}$ of $M_{1}$ with a reversal flow $\Phi_{2}$ which is outgoing along the boundary. Glue $M_{1}$ to $M_{2}$ along the boundary to produce closed manifold $M$ with flow $\Phi$. Franks and Williams show that $\Phi$ is Anosov and it is not transitive: it has a separating transverse torus.

Now construct the foliation transverse to $\Phi$. Start with the fibration in $M_{0}$ which is transverse to $\Phi_{0}$. This produces a fibration in $M_{1}$ which is transverse to the boundary torus $T$. Spin the fibers around the boundary torus $T$ and add $T$ as a torus leaf so that the foliation is still transverse to the flow. Then $M_{1}$ has a foliation transverse to $\Phi_{1}$. Put a similar foliation in $M_{2}$. The resulting foliation $\mathcal{F}$ in $M$ is transverse to the Anosov flow $\Phi$ but is not taut: it has a separating torus $T$. $\mathcal{F}$ is a depth one foliation with a unique compact leaf $T$ and is a fibration over the circle in any component of the complement of this compact leaf Ga1. There is no closed transversal through $T$. The gluing can be chosen so that $M$ fibers over the circle with fiber a surface of genus 2 and reducible monodromy, even though that is not necessary for the construction. 
In the example above notice that $\Phi$ is not regulating for $\mathcal{F}$, because there are closed orbits of $\Phi$ contained in $M_{1}$. Their lifts cannot intersect any lift of a leaf of $\mathcal{F}$ in $M_{2}$.

Still there is much one can say if $\mathcal{F}$ is not taut:

Proposition 2.3. Let $\Phi$ be a pseudo-Anosov flow transverse to a foliation $\mathcal{F}$. If $\mathcal{F}$ is not taut, then $\Phi$ is not regulating and $\mathcal{F}$ is not $\mathbf{R}$-covered.

Proof. Up to taking a finite cover of $M$, we may assume that $M$ is orientable and $\mathcal{F}$ is transversely orientable. If $\mathcal{F}$ is not taut, then Goodman Go showed that there are finitely many toral leaves $T_{1}, \ldots, T_{n}$ in $\mathcal{F}$ so that $T_{1} \cup \ldots \cup T_{n}$ bounds a submanifold $V$ and the flow is (say) incoming $V$ along $T_{1}, \ldots, T_{n}$. Any orbit intersecting $T_{1}$ is trapped inside $V$ in forward time and hence can only limit inside $V$ and any limit is an orbit $\gamma$ entirely contained in $V$. Then $\gamma \cap T_{i}=\emptyset$ for any $i$, so in the universal cover any lift $\widetilde{\gamma}$ will not intersect any lift of $T_{i}$. Therefore the flow $\Phi$ is not regulating for $\mathcal{F}$.

Now we show that $\mathcal{F}$ is not $\mathbf{R}$-covered. By theorem 2.1 $\mathcal{F}$ is Reebless. Suppose first that $n \geq 2$. There is no transversal to $\mathcal{F}$ through both $T_{1}$ and $T_{2}$, hence no transversal through any lifts of $T_{1}, T_{2}$ to $\widetilde{M}$. This shows that $\mathcal{F}$ is not $\mathbf{R}$-covered. The remaining case is that $n=1$ and $T_{1}$ bounds on both sides. Let $V$ be the closure of a component of $M-T_{1}$. If $\pi_{1}\left(T_{1}\right)$ surjects in $\pi_{1}(V)$, then since $\mathcal{F}$ is Reebless, $\pi_{1}(V) \cong \mathbf{Z} \oplus \mathbf{Z}$. This is impossible for a compact manifold $V$ with torus boundary; see chapter 10 of $\underline{\mathrm{He}}$. If $\pi_{1}\left(T_{1}\right)$ does not surject in $\pi_{1}(V)$, there is a closed loop $\alpha \in V$ not homotopic into $T_{1}$. Let $g$ be the covering translation of $\widetilde{M}$ associated to $\alpha$. In the universal cover $g\left(\widetilde{T}_{1}\right) \neq \widetilde{T}_{1}$. But $g\left(\widetilde{T}_{1}\right), \widetilde{T}_{1}$ do not cross a transversal, otherwise there would be a closed transversal to $T_{1}$ in $M$. We conclude that $\mathcal{F}$ is not $\mathbf{R}$-covered.

\section{TOPOLOGICAL THEORY OF PSEUdO-ANOSOV FLOWS}

Let $\Phi$ be a pseudo-Anosov flow in $M^{3}$ closed. We review the results about the topology of $\widetilde{\mathcal{F}}^{s}, \widetilde{\mathcal{F}}^{u}$ which will be used essentially in the next section. We refer to Fe5, Fe9 for detailed proofs and explanations.

Definition 3.1. Let $L$ be a leaf of $\widetilde{\mathcal{F}}^{s}$ or a line leaf of a leaf of $\widetilde{\mathcal{F}}^{s}$. Then a half leaf of $L$ is a connected component $A$ of $L-\gamma$, where $\gamma$ is any full orbit in $L$. The closed half leaf is $\bar{A}=A \cup \gamma$ and its boundary is $\partial A=\gamma$. If $\zeta$ is an open, relatively compact, connected subset of $\Theta(L)$, then it defines a flow band $L_{1}$ of $L$ by $L_{1}=\Theta^{-1}(\zeta)$. Let $\bar{L}_{1}$ be the closure of $L_{1}$ in $\widetilde{M}$. If $\zeta$ is an open segment in $\Theta(L)$, then $\Theta^{-1}(\zeta)$ is called a segment flow band of $L$.

Two important facts: First if $F \in \widetilde{\mathcal{F}}^{s}$ and $G \in \widetilde{\mathcal{F}}^{u}$, then $F$ and $G$ intersect in at most one orbit, a consequence of index computations for foliations in the plane. A second fact: Suppose that a leaf $F \in \widetilde{\mathcal{F}}^{s}$ intersects two leaves $G, H \in \widetilde{\mathcal{F}}^{u}$ and so does $L \in \widetilde{\mathcal{F}}^{s}$. Then $F, L, G, H$ define a rectangle in $\widetilde{M}$; see Figure 3(a). There are no singularities of $\widetilde{\Phi}$ in the interior of the rectangle and there is a product structure of $\widetilde{\mathcal{F}}^{s}$ and $\widetilde{\mathcal{F}}^{u}$ in the rectangle.

Definition 3.2. Perfect fits - Two leaves $F \in \widetilde{\mathcal{F}}^{s}$ and $G \in \widetilde{\mathcal{F}}^{u}$, form a perfect fit if $F \cap G=\emptyset$ and there are line leaves $F_{0}, G_{0}$ of $F, G$ respectively and half leaves $F_{1}$ of $F_{0}$ and $G_{1}$ of $G_{0}$ and also segment flow bands $L_{1} \subset L \in \widetilde{\mathcal{F}}^{s}$ and $H_{1} \subset H \in \widetilde{\mathcal{F}}^{u}$, 


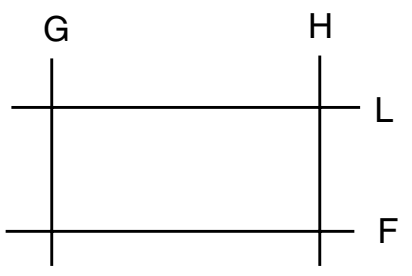

(a)

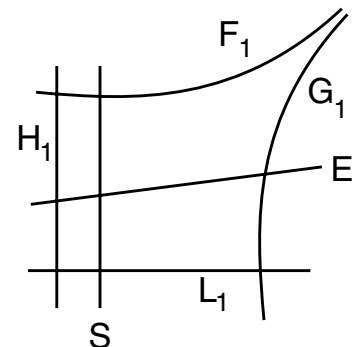

(b)

Figure 3. (a) Rectangles. (b) Perfect fits in the universal cover.

so that $F_{0}$ is regular on the side containing $L, G_{0}$ is regular on the side containing $H$ and

$$
\begin{gathered}
\bar{L}_{1} \cap \bar{G}_{1}=\partial L_{1} \cap \partial G_{1}, \quad \bar{L}_{1} \cap \bar{H}_{1}=\partial L_{1} \cap \partial H_{1}, \quad \bar{H}_{1} \cap \bar{F}_{1}=\partial H_{1} \cap \partial F_{1}, \\
\text { with } \bar{L}_{1} \cap \bar{G}_{1} \neq \emptyset, \quad \bar{L}_{1} \cap \bar{H}_{1} \neq \emptyset \text { and } \bar{H}_{1} \cap \bar{F}_{1} \neq \emptyset .
\end{gathered}
$$

Furthermore

$$
\begin{gathered}
\forall S \in \widetilde{\mathcal{F}}^{u}, \quad S \cap L_{1} \neq \emptyset \Rightarrow S \cap F_{1} \neq \emptyset \text { and } \\
\forall E \in \widetilde{\mathcal{F}}^{s}, \quad E \cap H_{1} \neq \emptyset \Rightarrow E \cap G_{1} \neq \emptyset .
\end{gathered}
$$

We refer to Figure [3(b) for perfect fits. Implications (1), (2) imply equivalences (that is, $S \cap L_{1} \neq \emptyset \Leftrightarrow S \cap F_{1} \neq \emptyset$ and the same for (2)). The set $\bar{F}_{1} \cup \bar{H}_{1} \cup \bar{L}_{1} \cup \bar{G}_{1}$ separates $\widetilde{M}$. Let $A$ be the complementary region which does not contain $F-F_{1}$ in its closure. Then there are no singularities of $\widetilde{\Phi}$ in $A$. Therefore perfect fits produce "ideal" rectangles, in the sense that even though $F$ and $G$ do not intersect, there is a product structure (of $\widetilde{\mathcal{F}}^{s}$ and $\widetilde{\mathcal{F}}^{u}$ ) in the interior of $A$.

Definition 3.3. Given $p \in \widetilde{M}$ (or $p \in \mathcal{O}$ ) and a half leaf $H$ of $\widetilde{W}^{u}(p)$ defined by $\widetilde{\Phi}_{\mathbf{R}}(p)$, let

$$
\mathcal{J}^{u}(H)=\left\{F \in \mathcal{H}\left(\widetilde{\mathcal{F}}^{s}\right) \mid F \cap H \neq \emptyset\right\} \subset \mathcal{H}\left(\widetilde{\mathcal{F}}^{s}\right) .
$$

Notice that $\widetilde{W^{s}}(p) \notin \mathcal{J}^{u}(H)$. Also let

$$
\mathcal{L}^{u}(H)=\bigcup\left\{p \in \widetilde{M} \mid p \in F \in \mathcal{J}^{u}(H)\right\} \subset \widetilde{M} .
$$

Then $\mathcal{L}^{u}(H) \subset \widetilde{M}$ and $\widetilde{W^{s}}(p) \subset \partial \mathcal{L}^{u}(H)$. Similarly define $\mathcal{J}^{s}(L), \mathcal{L}^{s}(L)$ for a stable half leaf $L$.

Definition 3.4. Lozenges - Let $p, q \in \widetilde{M}$ and half leaves $L_{p}, H_{p}$ of $\widetilde{W^{s}}(p), \widetilde{W}^{u}(p)$ defined by $\widetilde{\Phi}_{\mathbf{R}}(p)$, half leaves $L_{q}, H_{q}$ of $\widetilde{W}^{s}(q), \widetilde{W}^{u}(q)$ defined by $\widetilde{\Phi}_{\mathbf{R}}(q)$ so that

$$
\mathcal{L}^{u}\left(L_{p}\right) \cap \mathcal{L}^{s}\left(H_{q}\right)=\mathcal{L}^{u}\left(L_{q}\right) \cap \mathcal{L}^{s}\left(H_{p}\right) \subset \widetilde{M}
$$

Then this intersection is called a lozenge $\mathcal{A}$ in $\widetilde{M}$. The corners of the lozenge are $\widetilde{\Phi}_{\mathbf{R}}(p)$ and $\widetilde{\Phi}_{\mathbf{R}}(q)$ and $\mathcal{A}$ is a subset of $\widetilde{M}$. The sides of $\mathcal{A}$ are $L_{p}, H_{p}, L_{q}, H_{q}$. The sides are not contained in the lozenge, but are in the boundary of the lozenge.

Sometimes we also refer to $p$ and $q$ as corners of the lozenge. There are no singularities in the lozenges. This in fact shows that $\mathcal{A}$ is an open region in $\widetilde{M}$. 
(a)

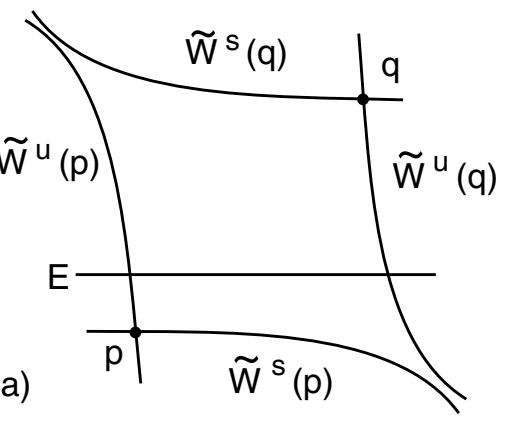

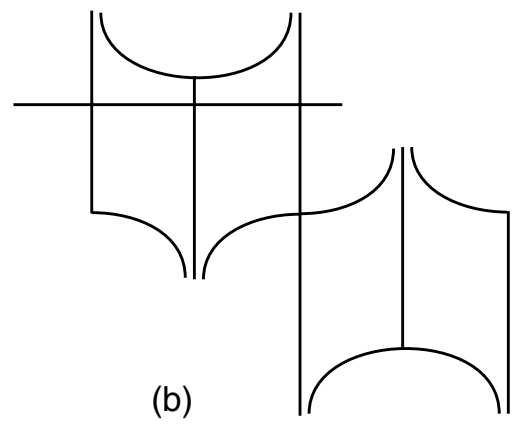

Figure 4. (a) A lozenge. (b) A chain of lozenges.

However there may be singular orbits on the sides of the lozenge and the corner orbits also may be singular. The definition of a lozenge implies that $L_{p}, H_{q}$ form a perfect fit and so do $L_{q}, H_{p}$. This is an equivalent way to define a lozenge with corners $\widetilde{\Phi}_{\mathbf{R}}(p), \widetilde{\Phi}_{\mathbf{R}}(q)$. Given any four leaves there is at most one lozenge with sides in them, so we may refer to the full leaves as the sides of the lozenge.

Two lozenges are adjacent if they share a corner and there is a stable or unstable leaf intersecting both of them; see Figure 4(b). Therefore they share a side. A chain of lozenges is a collection $\left\{\mathcal{A}_{i}\right\}, i \in I$, where $I$ is an interval (finite or not) in $\mathbf{Z}$; so that if $i, i+1 \in I$, then $\mathcal{A}_{i}$ and $\mathcal{A}_{i+1}$ share a corner; see Figure 4 (b). Consecutive lozenges may be adjacent or not. The chain is finite if $I$ is finite. We say that two orbits $\gamma, \alpha$ of $\widetilde{\Phi}$ (or the leaves $\left.\widetilde{W}^{s}(\gamma), \widetilde{W}^{s}(\alpha)\right)$ are connected by a chain of lozenges $\left\{\mathcal{A}_{i}\right\}, 1 \leq i \leq n$, if $\gamma$ is a corner of $\mathcal{A}_{1}$ and $\alpha$ is a corner of $\mathcal{A}_{n}$.

Definition 3.5. Suppose $\zeta \subset E \in \widetilde{\mathcal{F}}^{s}$ is a (possibly infinite) strong stable segment so that for each $p \in \zeta$ there is a half leaf $H_{p}$ of $\widetilde{W}^{u}(p)$ defined by $\widetilde{\Phi}_{\mathbf{R}}(p)$ so that

$$
\forall p, q \in \zeta, \quad \mathcal{J}^{u}\left(H_{p}\right)=\mathcal{J}^{u}\left(H_{q}\right) . \quad \text { In that case let } \mathcal{P}=\bigcup_{p \in \zeta} H_{p} .
$$

Then $\mathcal{P} \subset \widetilde{M}$ is called an unstable product region with base segment $\zeta$. The base segment is not uniquely determined by $\mathcal{P}$. Similarly define stable product regions.

A fundamental result is the following:

Theorem 3.6 ([Fe5, Fe9]). Let $\Phi$ be a pseudo-Anosov flow in $M^{3}$ closed and let $F_{0} \neq F_{1} \in \widetilde{\mathcal{F}}^{s}$. Suppose that there is a non-trivial covering translation $g$ with $g\left(F_{i}\right)=F_{i}, i=0,1$. Let $\alpha_{i}, i=0,1$, be the periodic orbits of $\widetilde{\Phi}$ in $F_{i}$ so that $g\left(\alpha_{i}\right)=\alpha_{i}$. Then $\alpha_{0}$ and $\alpha_{1}$ are connected by a finite chain of lozenges $\left\{\mathcal{A}_{i}\right\}, 1 \leq$ $i \leq n$, and $g$ leaves invariant each lozenge $\mathcal{A}_{i}$ as well as their corners.

A leaf $L$ of $\widetilde{\mathcal{F}}^{s}$ or $\widetilde{\mathcal{F}}^{u}$ is called periodic if there is a non-trivial covering translation $g$ of $\widetilde{M}$ with $g(L)=L$. This is equivalent to $\pi(L)$ having a periodic orbit of $\Phi$. In the same way an orbit $\gamma$ of $\widetilde{\Phi}$ is periodic if $\pi(\gamma)$ is a periodic orbit of $\Phi$. The main result concerning non-Hausdorff behavior in the leaf spaces of $\widetilde{\mathcal{F}}^{s}, \widetilde{\mathcal{F}}^{u}$ is the following theorem. 


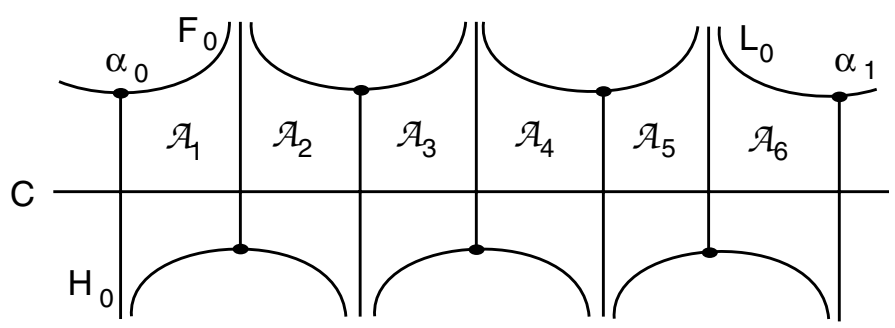

FiguRE 5. The correct picture between non-separated leaves of $\widetilde{\mathcal{F}}^{s}$.

Theorem 3.7 ([Fe5, [Fe9]). Let $\Phi$ be a pseudo-Anosov flow in $M^{3}$. Suppose that $F \neq L$ are not separated in the leaf space of $\widetilde{\mathcal{F}}^{s}$. Then $F$ is a periodic leaf and so is $L$. Let $F_{0}, L_{0}$ be the line leaves of $F, L$ which are not separated from each other. Let $V_{0}$ be the sector of $F$ bounded by $F_{0}$ and containing $L$. Let $\alpha$ be the periodic orbit in $F_{0}$ and $H_{0}$ be the component of $\left(\widetilde{W}^{u}(\alpha)-\alpha\right)$ contained in $V_{0}$. Let $g$ be a non-trivial covering translation with $g\left(F_{0}\right)=F_{0}, g\left(H_{0}\right)=H_{0}$ and $g$ leaves invariant the components of $\left(F_{0}-\alpha\right)$. Then $g\left(L_{0}\right)=L_{0}$. This produces closed orbits of $\Phi$ which are freely homotopic in $M$. Theorem 3.6 then implies that $F_{0}$ and $L_{0}$ are connected by a finite chain of lozenges $\left\{\mathcal{A}_{i}\right\}, 1 \leq i \leq n$, all contained in $\mathcal{L}^{u}\left(H_{0}\right)$ and all intersecting a common stable leaf $C$. Consecutive lozenges are adjacent. There is an even number of lozenges in the chain; see Figure 5 .

This result does not assume that $M$ is toroidal or atoroidal. In fact almost all Anosov flows satisfy this condition Fe9].

Finally we have the following result.

Theorem 3.8 ( $(\mathrm{Fe} 5, \mathrm{Fe} 9)$. Let $\Phi$ be a pseudo-Anosov flow in $M^{3}$. If there is a product region in $\widetilde{M}$, then $\Phi$ is an $\mathbf{R}$-covered pseudo-Anosov flow and $\Phi$ is topologically conjugate to a suspension Anosov flow. In particular $\mathcal{F}^{s}, \mathcal{F}^{u}$ do not have singularities. Furthermore any leaf of $\widetilde{\mathcal{F}}^{s}$ intersects every leaf of $\widetilde{\mathcal{F}}^{u}$ and vice versa.

\section{Regulating flows}

In this section we prove the main result of the article. First we obtain a useful property.

Proposition 4.1. Let $\Phi$ be a pseudo-Anosov flow transverse to an $\mathbf{R}$-covered foliation $\mathcal{F}$ and not regulating. Then for any leaf $G$ of $\widetilde{\mathcal{F}}$ there is an orbit $\gamma$ of $\widetilde{\Phi}$ not intersecting $G$. Conversely for any orbit $\alpha$ of $\widetilde{\Phi}$ there is a leaf $E$ of $\widetilde{\mathcal{F}}$ not intersected by $\alpha$.

Proof. Since $\Phi$ is not regulating for $\mathcal{F}$, let $\beta$ be orbit of $\widetilde{\Phi}$ and $H$ leaf of $\widetilde{\mathcal{F}}$, so that $\beta \cap H=\emptyset$. Without loss of generality assume that $\beta$ is in the back of $H$. Since $\mathcal{F}$ is Reebless, $\mathbf{R}$-covered and transverse to a pseudo-Anosov flow, proposition 2.3 implies that $\mathcal{F}$ is taut. Then we can choose finitely many closed transversals so that their union intersects every leaf of $\mathcal{F}$. Furthermore concatenating these transversals one finds a single closed transversal $\delta$ which intersects every leaf of $\mathcal{F}$. 
Now let $G$ be any leaf of $\widetilde{\mathcal{F}}$. Then $\delta$ intersects $\pi(H)$ and $\pi(G)$, leaves of $\mathcal{F}$. Lifting to the universal cover we produce a transversal arc to $\widetilde{\mathcal{F}}$ going from $H$ to $g(G)$ in the positive direction. Here $g(G)$ is a covering translate of $G$. Then $g(G)$ is in the front of $H$ and $\beta$ is in the back of $H$, hence $g(G) \cap \beta=\emptyset$. Consequently $G \cap g^{-1}(\beta)=\emptyset$. This proves the first assertion of the proposition.

As for the second assertion. Since $\Theta(H)$ does not contain $\Theta(\beta)$, then by proposition 2.2, there is a line leaf $l$ in $\mathcal{O}$, boundary component of $\Theta(H)$ with $l \times \mathbf{R}$ either containing $\beta$ or separating $\beta$ from $H$. Since $\beta$ is in the back of $H$, then proposition 2.2 shows that $l$ is a line leaf of $\widetilde{\mathcal{F}}_{\mathcal{O}}^{s}$. Let $L$ be a non-singular leaf of $\widetilde{\mathcal{F}}^{s}$, close to $l \times \mathbf{R}$ and not intersecting $H$. Let $W_{1}$ be the component of $\widetilde{M}-L$ not containing $H$.

$\underline{\text { Claim }}-\pi\left(W_{1}\right)=M$.

Otherwise $\pi\left(W_{1}\right)$ is open, $\mathcal{F}^{s}$ saturated and not $M$. Let $Z$ be a leaf of $\mathcal{F}^{s}$ in the boundary of $\pi\left(W_{1}\right)$. Since $Z$ is not compact it limits on a regular point $p$. Locally near $p$ one finds many distinct local sheets of $Z$. The definition of $W_{1}$ implies that in fact $Z$ intersects $\pi\left(W_{1}\right)$. But then $Z \subset \pi\left(W_{1}\right)$, a contradiction. This proves the claim.

Therefore given an arbitrary orbit $\alpha$ of $\widetilde{\Phi}$ let $h$ be a covering translation of $\widetilde{M}$ with $h(\alpha) \subset W_{1}$. Then $h(\alpha) \cap H=\emptyset$, so $\alpha \cap h^{-1}(H)=\emptyset$. This finishes the proof of the proposition.

We now prove the main theorem of the article:

Theorem 4.2 (Main theorem). Let $\Phi$ be a pseudo-Anosov flow transverse to $\mathcal{F}$, with $\Phi$ not an $\mathbf{R}$-covered Anosov flow. Then $\Phi$ is regulating for $\mathcal{F}$ if and only if $\mathcal{F}$ is $\mathbf{R}$-covered.

Proof. If $\Phi$ is regulating for $\mathcal{F}$, then clearly $\mathcal{F}$ is $\mathbf{R}$-covered, because any orbit of $\widetilde{\Phi}$ provides a homeomorphism from its points to the leaf space of $\widetilde{\mathcal{F}}$. Suppose from now on that $\mathcal{F}$ is $\mathbf{R}$-covered but $\Phi$ is not regulating for $\mathcal{F}$. We will show that $\Phi$ must be an $\mathbf{R}$-covered Anosov flow.

Put an order in the leaf space of $\widetilde{\mathcal{F}}$ as follows: $F<_{s} G$ if $F$ is in the back of $G$. The goal is to show that there is a unique transversal direction to $\widetilde{\mathcal{F}}^{s}$ or $\tilde{\mathcal{F}}^{u}$.

1. General construction for periodic orbits of $\widetilde{\Phi}$ -

For each periodic orbit of $\widetilde{\Phi}$ we produce a chain of leaves of $\widetilde{\mathcal{F}}$ escaping in $\widetilde{\mathcal{F}}$ and associated to that we produce a chain of leaves of $\widetilde{\mathcal{F}}^{s}$ escaping in $\widetilde{\mathcal{F}}^{s}$. This defines a direction transverse to $\widetilde{\mathcal{F}}^{s}$, which we later prove is unique.

Then let $\gamma$ be a periodic orbit of $\widetilde{\Phi}$, with $g(\gamma)=\gamma$ and $g \in \pi_{1}(M)$ a generator of the isotropy group of $\gamma$. By the previous proposition, $\gamma$ does not intersect every leaf of $\widetilde{\mathcal{F}}$, so let $F^{\prime} \in \widetilde{\mathcal{F}}$ with $F^{\prime} \cap \gamma=\emptyset$. For simplicity assume that $\gamma$ is on the back side of $F^{\prime}$. Since there are leaves of $\widetilde{\mathcal{F}}$ intersecting $\gamma$ and $\widetilde{\mathcal{F}}$ is $\mathbf{R}$-covered, there is a unique leaf $F$ with $F \cap \gamma=\emptyset, \gamma$ in the back of $F$ and for any $E \in \widetilde{\mathcal{F}}$ sufficiently close to $F$ and in the back of $F$ then $E \cap \gamma \neq \emptyset$. In other words $F$ is the least element in $\widetilde{\mathcal{F}}$ satisfying $F \cap \gamma=\emptyset$ and $\gamma$ is in the back of $F$. For the existence and uniqueness of $F$, the fact that $\mathcal{F}$ is $\mathbf{R}$-covered is essential, as this may not be true otherwise. Taking $g^{-1}$ if necessary we may assume that $g(F) \leq_{s} F$. Since $g(\gamma)=\gamma$ and $g(F) \cap \gamma=\emptyset$, the definition of $F$ implies that $g(F)=F$. 
Since $F \cap \gamma=\emptyset$ and $\gamma$ is in the back of $F$, there is a stable line leaf $l$ of $\widetilde{\mathcal{F}}_{\mathcal{O}}^{s}$ with $l \subset \partial \Theta(F)$ and either $\gamma \subset l \times \mathbf{R}$ or $l \times \mathbf{R}$ separates $\gamma$ from $F$. By the same reasoning as above $g(l \times \mathbf{R})=l \times \mathbf{R}$. Let $L=l \times \mathbf{R}$ and $V$ be the component of $\widetilde{M}-L$ not containing $F$.

The following technical fact is needed later:

Claim 1.1 - There is $F_{1}$ in $\widetilde{\mathcal{F}}$ with $\gamma$ in the back of $F_{1}$ and $\widetilde{W}^{u}(\gamma) \cap F_{1}=\emptyset$.

In order to prove that we go in the opposite direction to $F$, that is, in the region $V$.

Suppose first that $L$ intersects all leaves of $\widetilde{\mathcal{F}}^{u}$ which intersect $V$. In particular the leaf space of $\widetilde{\mathcal{F}}^{u}$ restricted to $V$ is homeomorphic to $\mathbf{R}$ and therefore $V$ does not contain singularities of $\widetilde{\Phi}$. Since $\pi(V)=M$ this implies that there are no singularities of $\Phi$, that is, $\Phi$ is an Anosov flow. Suppose there are $A, B \in \widetilde{\mathcal{F}}^{u}$ which are not separated from each other. Because $\pi(V)=M$, there is $h \in \pi_{1}(M)$ so that $h(B) \cap L \neq \emptyset$ and $h(A) \subset V$. But then $h(A) \cap L=\emptyset$ contradicting the above property of $L$. Hence the leaf space of $\widetilde{\mathcal{F}}^{s}$ is Hausdorff and $\Phi$ is an $\mathbf{R}$-covered Anosov flow. This contradicts the hypothesis of the theorem, so it cannot happen.

The remaining case is that there is a leaf $Z$ of $\widetilde{\mathcal{F}}^{u}$ with $Z \subset V$ and $Z \cap L=\emptyset$. We may assume that $Z$ is non-singular. Let $h_{1} \in \pi_{1}(M)$ with $h_{1}(\gamma)$ in the component of $\widetilde{M}-Z$ not intersecting $L$. Then $h_{1}\left(\widetilde{W}^{u}(\gamma)\right) \subset V$ and $h_{1}\left(\widetilde{W}^{u}(\gamma)\right)$ will not intersect any leaf $F^{\prime} \in \widetilde{\mathcal{F}}$ with $F^{\prime} \geq_{s} F$. So $\widetilde{W^{u}}(\gamma)$ will not intersect any leaf $\geq_{s} h_{1}^{-1}(F)$. This settles claim 1.1.

Now we construct the chains in $\widetilde{\mathcal{F}}$ and $\widetilde{\mathcal{F}}^{s}$. As done before let $F_{1}$ be the smallest leaf of $\widetilde{\mathcal{F}}$ equal to $F$ or in the front of $F$ and not intersecting $\widetilde{W}^{u}(\gamma)$. Since $g\left(\widetilde{W}^{u}(\gamma)\right)=\widetilde{W}^{u}(\gamma)$, then as above $g\left(F_{1}\right)=F_{1}$. Also $\gamma$ is in the back of $F_{1}$. Now there is $l_{1}$ a stable line leaf in $\partial \Theta\left(F_{1}\right)$ with either $\gamma$ in $l_{1} \times \mathbf{R}$ or $\gamma$ separated from $F_{1}$ by $l_{1} \times \mathbf{R}$. If $\gamma \subset \partial \Theta\left(F_{1}\right)$, then since $l_{1}$ is a stable line leaf it follows that $\widetilde{W}^{u}\left(\gamma_{1}\right) \cap F_{1} \neq \emptyset$. Then $\widetilde{W}^{u}(\gamma) \cap F_{1} \neq \emptyset$, a contradiction. So the second option occurs. Let $L_{1}=l_{1} \times \mathbf{R}$. Then $g\left(L_{1}\right)=L_{1}$, so there is an orbit $\gamma_{1}$ in $L_{1}$ with $g\left(\gamma_{1}\right)=\gamma_{1}$.

We can iterate this process: As before produce $F_{2}$ smallest in $\widetilde{\mathcal{F}}$ with $\gamma_{1}$ in the back of $F_{2}$ and $\widetilde{W}^{u}\left(\gamma_{1}\right) \cap F_{2}=\emptyset$. Then $g\left(F_{2}\right)=F_{2}$ and produce $l_{2}$ stable line leaf $l_{2} \subset \partial \Theta\left(F_{2}\right)$ and $L_{2}=l_{2} \times \mathbf{R}$ with $g\left(L_{2}\right)=L_{2}$. In this way we produce $\gamma_{i}$ orbits of $\widetilde{\Phi}, F_{i}$ leaves of $\widetilde{\mathcal{F}}, L_{i}$ leaves of $\widetilde{\mathcal{F}}^{s}$ with

$$
g\left(\gamma_{i}\right)=\gamma_{i}, \quad g\left(F_{i}\right)=F_{i}, \quad g\left(L_{i}\right)=L_{i}, \quad F_{i}<_{s} \quad F_{i+1} \quad \text { and } g\left(\widetilde{W}^{u}\left(\gamma_{i}\right)\right) \cap F_{i+1}=\emptyset
$$

for all $i \in \mathbf{N}$; see Figure 6. In addition for all $i \in \mathbf{N}, L_{i+1}$ separates $L_{i}$ from $L_{i+2}$, that is, the $L_{i}$ are nested in $\widetilde{M}$.

Then the $F_{i}$ escapes in $\widetilde{M}$ when $i \rightarrow \infty$. Otherwise $F_{i} \rightarrow F^{*}$ unique leaf of $\tilde{\mathcal{F}}$ since $\mathcal{F}$ is $\mathbf{R}$-covered. As the family $l_{i}=\Theta\left(L_{i}\right) \subset \mathcal{O}, i \in \mathbf{N}$, is nested and trapped by $\Theta(F)$, then the $l_{i}$ also converge. There may be more than one limit as the leaf space of $\widetilde{\mathcal{F}}_{\mathcal{O}}^{s}$ may fail to be Hausdorff, but nevertheless there is a unique limit $l^{*}$ line leaf of $\widetilde{\mathcal{F}}_{\mathcal{O}}^{s}$ which separates $\Theta\left(F^{*}\right)$ from all $\Theta\left(L_{i}\right)$. Let $L^{*}=l^{*} \times \mathbf{R}$. Then $g\left(L_{i}\right)=L_{i}$ and $g\left(F^{*}\right)=F^{*}$ implies that $g\left(L^{*}\right)=L^{*}$ so there is a periodic orbit $\gamma^{*} \subset L^{*}$ with $g\left(\gamma^{*}\right)=\gamma^{*}$. Then $g\left(\widetilde{W}^{u}\left(\gamma^{*}\right)\right) \cap L_{i} \neq \emptyset$, for big enough $i$, producing two orbits 


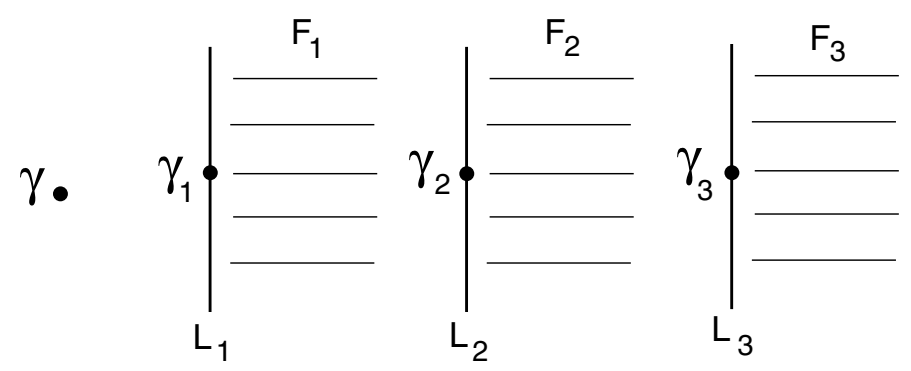

Figure 6 . The sequence of leaves invariant under $g$.

invariant by $g$ in $\widetilde{W}^{u}\left(\gamma^{*}\right)$, namely $\widetilde{W}^{u}\left(\gamma^{*}\right) \cap L_{i}$ and $\gamma^{*}$. This is a contradiction. Hence the $F_{i}$ escapes in $\widetilde{M}$.

We stress that the construction above works for any periodic orbit $\gamma$ of $\widetilde{\Phi}$. The point here is the following: The general construction suggests there is a standard "direction" in the universal cover "transverse" to $\widetilde{\mathcal{F}}^{s}$ which is created by the increasing $F_{i}$. This is the direction of the nested $L_{i}$. The following property shows that this "direction" given by the $L_{i}$ is essentially independent of the periodic orbit $\gamma$. This will eventually force the leaf space of $\widetilde{\mathcal{F}}^{s}$ (or $\widetilde{\mathcal{F}}^{u}$ ) to be non-singular and Hausdorff proving the theorem.

\section{Nesting property -}

If $\alpha$ is another periodic orbit of $\widetilde{\Phi}$ associated to the covering translation $f$ of $\widetilde{M}$, the general construction above produces sequences $\alpha_{i}$ of periodic orbits of $\widetilde{\Phi}, G_{i}$ leaves of $\widetilde{\mathcal{F}}$ and $E_{i}$ line leaves of $\widetilde{\mathcal{F}}^{s}$ which are all invariant under $f$ for any $i \in \mathbf{N}$.

Claim 2.1 - The nested sequence $\left\{E_{i}\right\}, i \in \mathbf{N}$, is eventually nested with the sequence $\left\{L_{j}\right\}, j \in \mathbf{N}$.

This means that for any $i$ big enough, there are $j, k$ so that $E_{i}$ separates $L_{j}$ from $L_{k}$ and vice versa. This will prove there is a unique "up" direction transverse to $\widetilde{\mathcal{F}}^{s}$.

To prove the claim, first notice that $G_{i} \in \widetilde{\mathcal{F}}$ also has to escape in $\widetilde{M}$ when $i \rightarrow \infty$. Hence there is $i_{0} \in \mathbf{N}$ so that $G_{i_{0}} \geq_{s} F_{j_{0}}$ for some $j_{0} \in \mathbf{N}$. Given $i>i_{0}$ there is a unique $\left.j(i) \in \mathbf{N}\left(j(i) \geq j_{0}\right)\right)$ so that either $G_{i}=F_{j(i)}$ or $F_{j(i)} \leq_{s} G_{i}<_{s} F_{j(i)+1}$. This shows that the sequences $\left\{F_{k}\right\}, k \in \mathbf{N}$, and $\left\{G_{k}\right\}, k \in \mathbf{N}$, are eventually nested. We want to show the same thing for the $\left\{L_{k}\right\},\left\{E_{k}\right\}, k \in \mathbf{N}$.

Fix $i$ and let $j=j(i)$. Consider $L_{j}, L_{j+1}$ and the 3 types of components of $\widetilde{M}-\left(L_{j} \cup L_{j+1}\right)$ :

- $W_{1}$ is a component not intersecting $F_{j}$ or $F_{j+1}$, essentially "below" $F_{j}$ - there is more than one such component if $L_{j}$ is a singular leaf,

- $W_{2}$ is the component between $L_{j}$ and $L_{j+1}$. Equivalently the one which has both $L_{j}$ and $L_{j+1}$ in its boundary. This is unique.

- $W_{3}$ is a component not containing $L_{j}$ but with a line leaf of $L_{j+1}$ in its boundary; see Figure 7(a). 


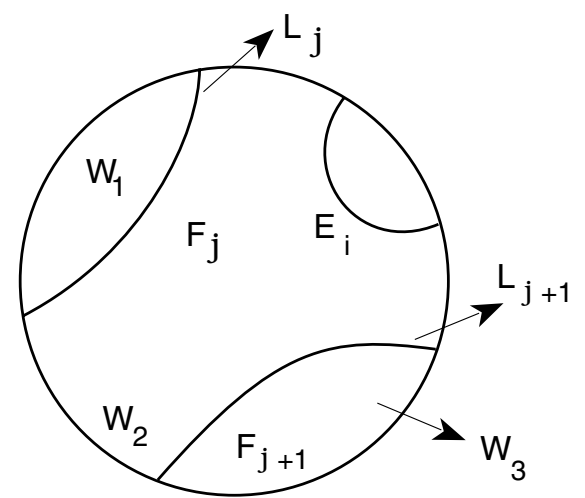

(a)

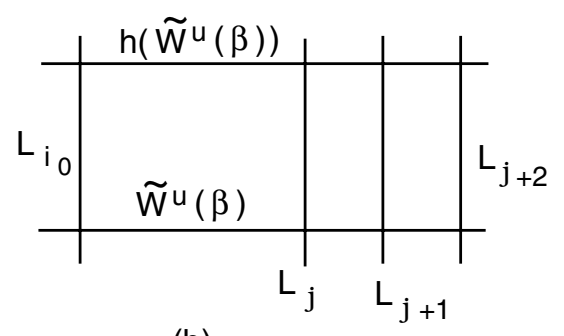

(b)

Figure 7. (a) The nesting between $E_{i}$ and $L_{j}$. (b) Producing a product region in $\widetilde{M}$.

For simplicity we consider the situation where both $L_{j}$ and $L_{j+1}$ are non-singular. In this case $W_{1}, W_{3}$ are unique. In general $\widetilde{M}-\left(L_{j} \cup L_{j+1}\right)$ may have more components, but the same arguments apply.

We claim that $E_{i}$ has to be either $L_{j}$ or to separate $L_{j}$ from $L_{j+1}$, so it must be in the closure of $W_{2}$ in $\widetilde{M}$. If for instance $E_{i} \subset W_{1}$, then as $\Theta\left(G_{i}\right)$ has a boundary component in $\Theta\left(E_{i}\right)$, then $G_{i}$ has points in $W_{1}$, too. This forces $G_{i}<_{s} F_{j}$, a contradiction. If on the other hand $E_{i} \subset W_{3}$, then $G_{i}>_{s} F_{j+1}$. We conclude that $E_{i}$ is in the closure of $W_{2}$. Assume that $E_{i} \neq L_{j}, L_{j+1}$, for otherwise we are done. If $E_{i}$ does not separate $L_{j}$ from $L_{j+1}$, then $E_{k}, k>i$, will be contained in the component of $\widetilde{M}-E_{i}$ not containing $L_{j+1}$; see Figure 7 (a). This is a contradiction because $G_{k}>F_{j+1}$ for some $k$. We conclude that $E_{i}$ separates $L_{j}$ from $L_{j+1}$. This proves claim 2.1. This is the nesting property we are looking for.

In addition suppose the $\left\{L_{i}\right\}, i \in \mathbf{N}$, are line leaves of $\widetilde{\mathcal{F}}^{s}$ of any such sequence given by the general construction. Then $\left\{L_{i}\right\}, i \in \mathbf{N}$, escapes in $\widetilde{M}$ as $i \rightarrow \infty$. Otherwise $L_{i} \rightarrow L^{*}$ line leaf of $\widetilde{\mathcal{F}}^{s}$ and maybe other limits as well since the leaf space of $\widetilde{\mathcal{F}}^{s}$ may fail to be Hausdorff. Let $V_{2}$ be the component of $\widetilde{M}-L^{*}$ not containing the $L_{i}$. Then $\pi\left(V_{2}\right)=M$ so let $h$ be a covering translation so that $h\left(L^{*}\right) \subset V_{2}$. For $j$ big enough $h\left(L_{j}\right) \subset V_{2}$ and cannot be nested with the sequence $L_{i}, i \in \mathbf{N}$. This fails the nesting property and is therefore not possible. Therefore we can now conclude that the sequence $\left\{L_{i}\right\}, i \in \mathbf{N}$, escapes in $\widetilde{M}$.

The general construction and the nesting property will imply that starting in any periodic orbit in $\widetilde{M}$ there is a chain of lozenges going to the "canonical" direction of the $L_{i}, i \in \mathbf{N}$. By carefully choosing where to put the initial periodic orbits we will show this is incompatible with the existence of singularities of $\widetilde{\Phi}$ or non-Hausdorff behavior in the leaf space of $\widetilde{\mathcal{F}}^{s}$ or $\widetilde{\mathcal{F}}^{u}$.

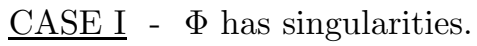

We will show this does not happen. By way of contradiction, let $\beta$ be a singular orbit of $\widetilde{\Phi}$. Consider one chain $\gamma_{i}, L_{i}, F_{i}, i \in \mathbf{N}$, given by the general construction, 
all invariant under $g \in \pi_{1}(M)$. Since the sequence $\left\{L_{i}\right\}, i \in \mathbf{N}$, escapes in $\widetilde{M}$ and are nested, it follows that the $L_{i}$ are eventually in a fixed sector $V_{0}$ of $\widetilde{W}^{s}(\beta)$.

Suppose first that all leaves $L_{i}, i \in \mathbf{N}$, intersect $\widetilde{W}^{u}(\beta)$. Let $h \in \pi_{1}(M)$. The sequences of leaves $h\left(\gamma_{i}\right), h\left(L_{i}\right), h\left(F_{i}\right), i \in \mathbf{N}$, are those obtained by the general construction if one starts with the orbit $h(\gamma)$. By the nesting property above, the $\left\{h\left(L_{j}\right)\right\}, j \in \mathbf{N}$, are eventually nested with the $\left\{L_{i}\right\}, i \in \mathbf{N}$. Since $h\left(\widetilde{W}^{u}(\beta)\right)$ intersects all of $h\left(L_{i}\right)$, it follows that $h\left(\widetilde{W}^{u}(\beta)\right)$ will intersect all $L_{i}, i \geq i_{0}$, for some $i_{0}$ big enough. Choose $h$ so that $h\left(\widetilde{W}^{u}(\beta)\right) \neq \widetilde{W}^{u}(\beta)$. For any $j>i_{0}$, the leaves $\widetilde{W}^{u}(\beta), h\left(\widetilde{W}^{u}(\beta)\right), L_{i_{0}}, L_{j}$ form a rectangle in $\widetilde{M}$, hence there is a product structure in the interior of the rectangle. Let $V_{1}$ be the component of $\widetilde{M}-L_{i_{0}}$ containing $L_{i}$ for $i>i_{0}$. Since $L_{j}$ escapes in $\widetilde{M}$ as $j \rightarrow \infty$, it follows that any leaf unstable leaf of $\widetilde{\mathcal{F}}^{u}$ between $\widetilde{W}^{u}(\beta)$ and $h\left(\widetilde{W}^{u}(\beta)\right)$ intersects the same set of stable leaves restricted to $V_{1}$. This creates a stable product region contained in $V_{1}$ and with base segment in $L_{i_{0}}$; see Figure 7 (b). By theorem 3.8 the foliations $\widetilde{\mathcal{F}}^{s}, \widetilde{\mathcal{F}}^{u}$ have leaf space homeomorphic to $\mathbf{R}$. This contradicts the existence of singularities.

Therefore we may assume that $L_{i} \cap \widetilde{W}^{u}(\beta)=\emptyset$ for $i$ big enough and therefore the $L_{i}$ are eventually in a single component $C$ of $Y=\widetilde{M}-\left(\widetilde{W}^{u}(\beta) \cup \widetilde{W}^{s}(\beta)\right)$. Since there at least 3 prongs in $\widetilde{W}^{s}(\beta), \widetilde{W}^{u}(\beta)$, there are at least 6 components of $Y$. Let $D$ be a component of $Y$ which is separated from $C$ by at least 3 separatrices on each side. For simplicity we analyse the case that $\widetilde{W}^{s}(\beta), \widetilde{W}^{u}(\beta)$ have 3 prongs; see Figure $8(a)$.

The proof now will go as follows: start with a periodic orbit in $D$ and use the general sequence. This eventually has to go into $C$, which will be a contradiction.

First consider $\pi(D) \subset M$. If this is not $M$, then it is a region whose boundary may have a corner, coming from $\beta$ and is otherwise saturated by leaves of $\mathcal{F}^{s}$ or $\mathcal{F}^{u}$. In a similar way to a previous argument, this is impossible, therefore $\pi(D)=M$. Hence there are periodic orbits of $\widetilde{\Phi}$ in $D$. Let $\alpha$ be one such periodic orbit and $f$ a non-trivial covering tranlation of $\widetilde{M}$ generating the isotropy group of $\alpha$. The general construction produces sequences $\alpha_{i}$ periodic orbits of $\widetilde{\Phi}, E_{i}$ line leaves of $\widetilde{\mathcal{F}}^{s}$ and $G_{i}$ leaves of $\widetilde{\mathcal{F}}, i \in \mathbf{N}$, all invariant under $f \in \pi_{1}(M)$ associated to $\alpha$. By the nesting property the sequence $E_{i}, i \in \mathbf{N}$, is eventually nested with $L_{i}, i \in \mathbf{N}$. Therefore both sequences are eventually contained in $C$. Let $i_{0}$ with $L_{i_{0}} \subset C$. Then $f(\alpha)=\alpha$ and $f\left(\alpha_{i_{0}}\right)=\alpha_{i_{0}}$. By theorem 3.6 there is a chain of lozenges $\mathcal{B}_{k}, 1 \leq k \leq n$, from $\alpha$ to $\alpha_{i_{0}}$. Let $\delta_{k}, 0 \leq k \leq n$, be the periodic orbits which are the corners of these lozenges, so $\delta_{0}=\alpha, \delta_{n}=\alpha_{i_{0}}$. The goal is to show that any such sequence of lozenges has to have a corner in $\beta$ which will turn out to be impossible.

Let $D_{1}, D_{2}, D_{3}, D_{4}$ be the other components of $Y$ as described in Figure 8 (a). Also let $S_{1}, S_{2}, S_{3}$ be the components of $\widetilde{W}^{s}(\beta)-\beta$ as described in Figure 8 (a) and let $U_{1}, U_{2}, U_{3}$ be the components of $\widetilde{W}^{u}(\beta)-\beta$. These are the separatrices of $Y$. The chain of lozenges has to go from $D$ to $C$ so it has to intersect the boundary of $D$. For simplicity suppose that $\delta_{0}$ is the last corner in the chain which is contained in $D$. Therefore $\mathcal{B}_{1}$ has to intersect the boundary of $D$. Suppose first that it crosses the stable boundary of $D$, namely $S_{1}$. Here the term cross means that the lozenge $\mathcal{B}_{1}$ intersects $S_{1}$ in the interior. Therefore an unstable side $Q_{1}$ of $\mathcal{B}_{1}$ crosses the interior of $S_{1}$ into the adjacent component $D_{1}$; see Figure 8 (a). In order for the 

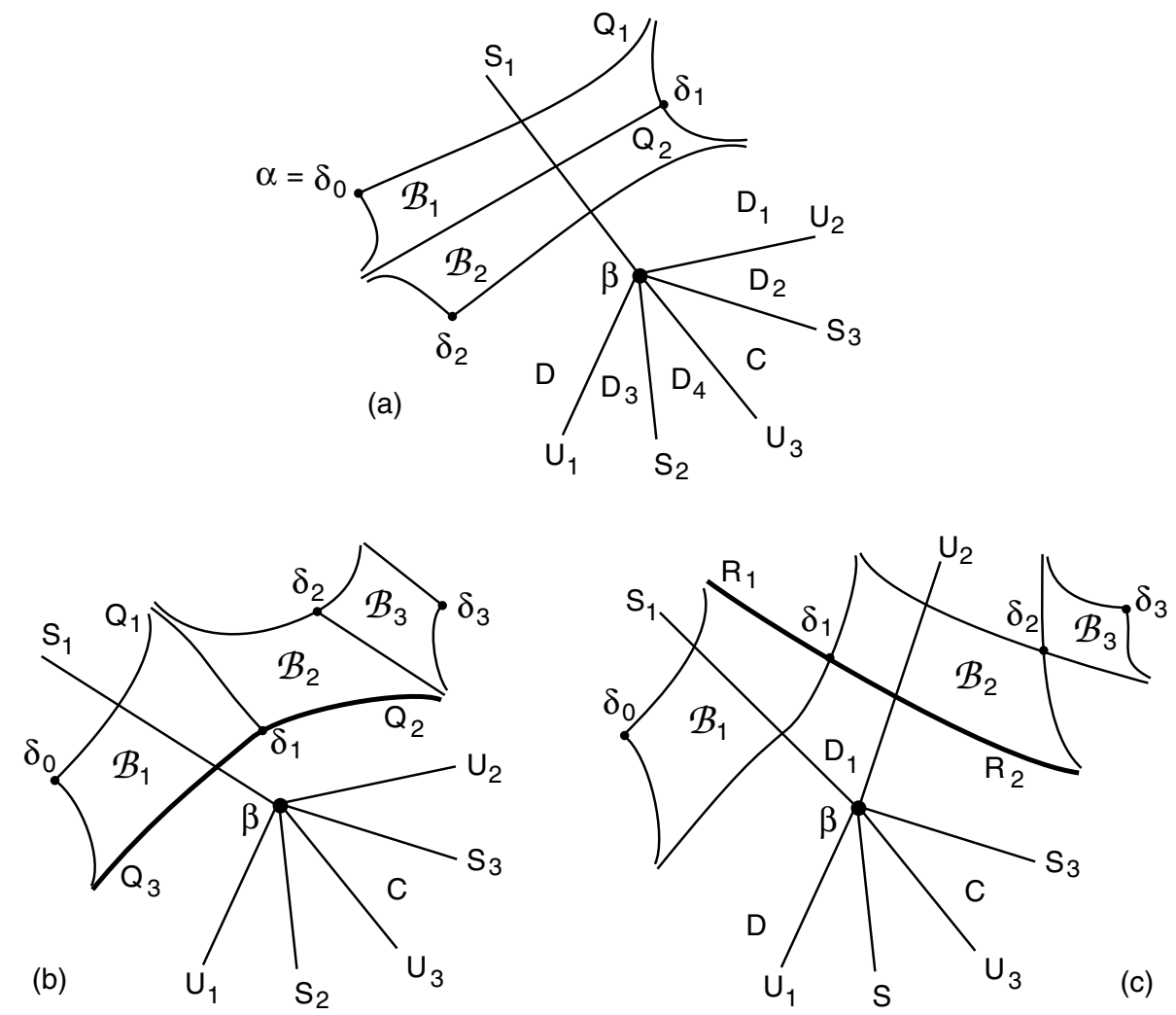

Figure 8. (a) Separating components of $Y$ and first option. (b) The union $Q_{1} \cup Q_{2}$ traps lozenges away from $C$. (c) The union $R_{1} \cup R_{2}$ traps lozenges away from $C$.

lozenge to close up, the other corner of $\mathcal{B}_{1}$ has to be in $D_{1}$; see Figure 8 (a). Consider the second lozenge $\mathcal{B}_{2}$. If it is adjacent to $\mathcal{B}_{1}$ along an unstable side $Q_{2}$, this forces the corner $\delta_{2}$ to be contained in $D$; see Figure 8 (a). This contradicts the choice of $\delta_{0}$ as the only corner in $D$. Suppose $\mathcal{B}_{2}$ is adjacent to $\mathcal{B}_{1}$ along a stable side; see Figure $8(\mathrm{~b})$. Then there is an unstable side $Q_{2}$ of $\mathcal{B}_{2}$ and an unstable side $Q_{3}$ of $\mathcal{B}_{1}$, so that $Q_{2}$ and $Q_{3}$ belong to the same unstable leaf $Q$; see Figure 8 (b). The leaf $Q$ separates the lozenges $\mathcal{B}_{1}, \mathcal{B}_{2}$ from $C$ and all further lozenges in the chain are contained in the same component of $\widetilde{M}-Q$. Therefore the chain can never reach $C$, a contradiction. The same occurs if $\mathcal{B}_{2}$ does not immediately cross to the next component $D_{2}$. The final possibility is that the other corner of $\mathcal{B}_{2}$ is in $D_{2}$, that is, $\mathcal{B}_{2}$ crosses the unstable standard leaf $U_{2} \subset \widetilde{W}^{u}(\beta)$; see Figure $8(\mathrm{c})$. Then there is a stable side $R_{1}$ of $\mathcal{B}_{1}$ and a stable side $R_{2}$ of $\mathcal{B}_{2}$ which are in the same stable leaf $R$ of $\widetilde{\mathcal{F}}^{s}$; see Figure 8 (c). The leaf $R$ separates $\mathcal{B}_{2}$ from $C$ and will also separate all further lozenges in the chain from the set $C$. Again this is a contradiction.

We conclude that the chain cannot cross $S_{1}$. In the same way the chain cannot cross $U_{1}$. 
The point here is that the chain of lozenges can only cross at most two separatrices of $Y$ but not 3. Since $D$ is separated from $C$ by at least 3 separatrices in each side, there is no way to go from $D$ to $C$ crossing the interior of the separatrices.

The remaining option is that one of the corners in the chain is actually $\beta$ and $\mathcal{B}_{1}$ has sides in $S_{1}$ and $U_{1}$ rather than crossing these standard leaves. Then $\mathcal{B}_{2}$ could jump to the other side and be contained in $C$, without the chain having to cross the interior of the separatrices. A priori there is no contradiction. But since this has to happen for any periodic orbit in $D$ then any periodic orbit in $D$ has to be connected to $\beta$ by a chain of lozenges. If the set of periodic orbits in $D$ projects to an infinite collection of closed orbits in $M$, then they have to limit somewhere in $M$ and so in $\widetilde{M}$ also. Therefore there will be two periodic orbits $\zeta_{1}, \zeta_{2}$ in $D$ so that $\widetilde{W}^{u}\left(\zeta_{1}\right) \cap \widetilde{W}^{s}\left(\zeta_{2}\right) \neq \emptyset$. Let $g_{0}$ be the generator of the isotropy group of $\beta$. As both $\zeta_{1}$ and $\zeta_{2}$ are connected to $\beta$ by a chain of lozenges, then $g_{0}\left(\zeta_{1}\right)=\zeta_{1}, g_{0}\left(\zeta_{2}\right)=\zeta_{2}$. This would produce two periodic orbits in $\widetilde{W}^{u}\left(\zeta_{1}\right)$, a contradiction. Consequently the collection of periodic orbits in $D$ projects to a finite collection of orbits in $M$. Since $\pi(D)=M$ this would imply there are only finitely many closed orbits of $\Phi$ in $M$. This is a contradiction Mo1, Man].

We conclude that case I cannot happen. Therefore $\Phi$ does not have singularities.

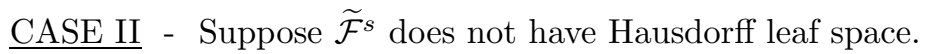

Now we know there are no singular orbits in $\Phi$. The general thrust of the proof will be similar to Case I. By hypothesis there are $A_{1}, A_{2} \in \widetilde{\mathcal{F}}^{s}$ which are not separated in their leaf space. By theorem $3.7 A_{1}, A_{2}$ are connected by a chain of adjacent lozenges all intersecting a common stable leaf. There are an even number of lozenges. For simplicity we may assume there are two lozenges $\mathcal{C}_{1}, \mathcal{C}_{2}$ in the chain. A stable leaf intersects $\mathcal{C}_{1}$ if and only if it intersects $\mathcal{C}_{2}$. Let $\xi_{1}, \xi_{2}$ be the periodic orbits in $A_{1}, A_{2}$, respectively. There are some special leaves for us: $A_{1}, A_{2}$ are the line leaves of $\widetilde{\mathcal{F}}^{s}$ not separated from each other. $A_{3}$ is the line leaf in the boundary of the union of the two lozenges $\mathcal{C}_{1} \cup \mathcal{C}_{2}$ - that is, contains the union of the stable sides of $\mathcal{C}_{1}, \mathcal{C}_{2}$ which are not in $A_{1}, A_{2}$. Finally $B_{1}\left(B_{2}\right)$ is the component of $\widetilde{W}^{u}\left(\xi_{1}\right)-\xi_{1}\left(\widetilde{W}^{u}\left(\xi_{2}\right)-\xi_{2}\right)$ which is a side of one of the lozenges $\mathcal{C}_{1}$ or $\mathcal{C}_{2}$; see Figure 9(a). Let $A_{1}^{\prime}$ be the component of $A_{1}-\xi_{1}$ not in the boundary of $\mathcal{C}_{1}$ and similarly define $A_{2}^{\prime}$. This defines 5 regions of $\widetilde{M}$ which will be important for us:

- Region (1) - the component of $\widetilde{M}-A_{1}$ not containing $A_{2}$,

- Region (2) - the component of $\widetilde{M}-A_{2}$ not containing $A_{1}$,

- Region (3) - the component of $\widetilde{M}-A_{3}$ not containing $A_{1}$,

- Region (4) - the component of $\widetilde{M}-\left(A_{1}^{\prime} \cup B_{1} \cup \xi_{1}\right)$ not containing $A_{2}$,

- Region (5) - the component of $\widetilde{M}-\left(A_{2}^{\prime} \cup B_{2} \cup \xi_{2}\right)$ not containing $A_{1}$.

Notice that $\widetilde{M}$ is the union of these 5 regions, plus the two lozenges and their sides, plus $A_{1}, A_{2}$; see Figure 9(a).

Let $\gamma$ be a periodic orbit of $\widetilde{\Phi}$ and the sequences $\gamma_{i}$ orbits of $\widetilde{\Phi}, L_{i}$ line leaves of $\widetilde{\mathcal{F}}^{s}$ and $F_{i} \in \widetilde{\mathcal{F}}$ as given by the general construction. As seen in the beginning of the proof of Part I, the $L_{i}$ cannot keep intersecting any of the leaves $B_{1}$ or $B_{2}$, or also the leaves $A_{1}, A_{2}, A_{3}$ since these last ones are stable leaves. Therefore the $L_{i}, i \in \mathbf{N}$, are eventually contained in one of the 5 regions defined above.

We analyse each possibility in turn. 


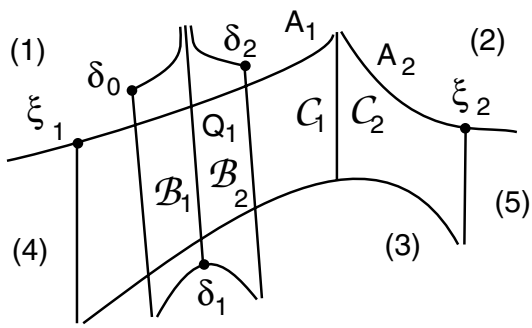

(a)

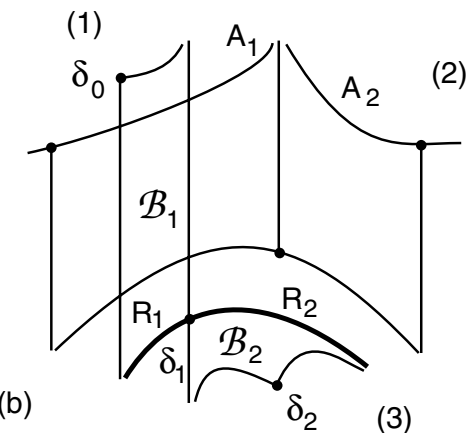

(3)

Figure 9. (a) Non-separated leaves in $\widetilde{\mathcal{F}}^{s}$ and regions of $\widetilde{M}$ associated to them. Trapping back and forth of corners of lozenges producing a contradiction. (b) The union $R_{1} \cup R_{2}$ separates $\mathcal{B}_{2}$ from $A_{2}$.

Case II.1 - Suppose the $L_{i}$ are eventually contained in region (2).

Start with a periodic orbit $\alpha$ in region (1). The general construction produces orbits $\alpha_{i}$ of $\widetilde{\Phi}$, line leaves $E_{i}$ of $\widetilde{\mathcal{F}}^{s}$ and leaves $G_{i} \in \widetilde{\mathcal{F}}, \forall i \in \mathbf{N}$. These are all invariant under $f \in \pi_{1}(M)$ associated to $\alpha$. By the nesting property the $E_{i}$ are eventually nested with the $L_{i}$ and so eventually in region (2). As before this produces a chain of lozenges $\mathcal{B}_{k}, 1 \leq k \leq n$, from $\delta_{0}=\alpha$ to $\delta_{n}=\alpha_{i_{0}}$, with $\alpha_{i_{0}} \subset$ region (2). Let $\delta_{j}, 0 \leq j \leq n$, be the corner orbits in this chain. Assume $\delta_{0}$ is the last corner in region (1). Then $\mathcal{B}_{1}$ has to intersect $A_{1}$. Assume first that the interior of $\mathcal{B}_{1}$ intersects $A_{1}$. If the second lozenge is adjacent to $\mathcal{B}_{1}$ along the common unstable side $Q_{1}$, then $Q_{1}$ intersects $A_{1}$ and this forces $\delta_{2}$ to be contained in region (1); see Figure 9(a). This contradicts the choice of $\delta_{0}$. The other option is that there are stable sides $R_{1}$ of $\mathcal{B}_{1}$ and $R_{2}$ of $\mathcal{B}_{2}$ contained in the stable leaf $\widetilde{W}^{s}\left(\delta_{1}\right)$ and so that $R_{1} \cup R_{2}$ separates $\mathcal{B}_{2}$ from $A_{1}$ and hence from $A_{2}$; see Figure 9(b). Then $R_{1} \cup R_{2}$ separates all further lozenges from $A_{2}$ and $A_{2}$ cannot be reached by the chain, a contradiction. The last option is that the chain has a corner in the periodic orbit $\xi_{1}$ in $A_{1}$. But then all periodic orbits in region (1) would have to be connected to this orbit by a chain of lozenges. As seen in the proof of case I, this is not possible either. We conclude that this case cannot happen.

If the sequence $L_{i}, i \in \mathbf{N}$, is eventually in region (1), then we switch the roles of regions (1) and (2) and apply the same proof.

Case II.2 - The $L_{i}, i \in \mathbf{N}$, are eventually in region (3).

In this case we let $\alpha$ be a periodic orbit contained in region (4). There is a chain of lozenges $\mathcal{B}_{k}, 1 \leq k \leq n$, with corners $\delta_{k}, 0 \leq k \leq n$, from $\alpha$ to $\alpha_{i_{0}} \subset$ region (3). As before assume $\delta_{0}$ is the last corner in region (4). Assume first that the interior of $\mathcal{B}_{1}$ intersects the unstable leaf $\widetilde{W}^{u}\left(\xi_{1}\right)$ - notice $B_{1} \subset \widetilde{W}^{u}\left(\xi_{1}\right)$. Then there is a stable side $R_{0}$ of $\mathcal{B}_{1}$ which intersects $B_{1} \subset \widetilde{W}^{u}\left(\xi_{1}\right)$ because $\delta_{0} \subset$ region (4). Since the lozenges $\mathcal{C}_{1}, \mathcal{C}_{2}$ are adjacent, this forces $R_{0}$ to also intersect $\mathcal{C}_{2}$ and enter region (5). Hence $\delta_{1}$ is contained in region (5). If $\mathcal{B}_{2}$ is adjacent to $\mathcal{B}_{1}$ along a stable side $R_{1}$, then this forces $\mathcal{B}_{2}$ to cross back to region (4) and $\delta_{2} \subset$ region (4); see Figure 10(a). This contradicts the choice of $\delta_{0}$. Otherwise there is an unstable side 


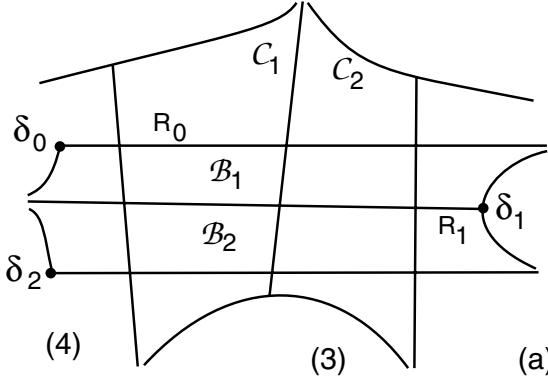

(a)

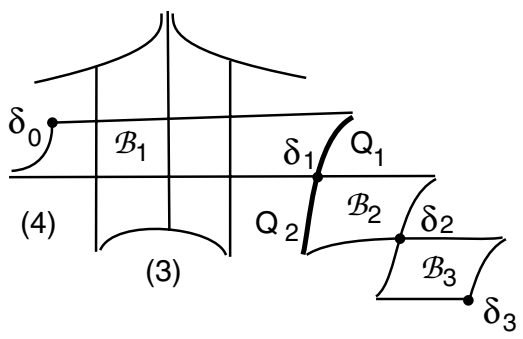

(b)

Figure 10. (a) Trapping back and forth of lozenges. (b) Separating leaf which traps lozenges.

$Q_{1}$ of $\mathcal{B}_{1}$ and an unstable side $Q_{2}$ of $\mathcal{B}_{2}$ so that $Q_{1} \cup Q_{2} \subset \widetilde{W}^{u}\left(\delta_{1}\right)$ and $Q_{1} \cup Q_{2}$ separates $\mathcal{B}_{2}$ from region (3); see Figure 10(b). In fact $Q_{1} \cup Q_{2}$ will separate all further lozenges from region (3), so $\alpha_{i_{0}}$ can never be achieved, a contradiction. The remaining option is that $\mathcal{B}_{1}$ has a side in $\widetilde{W}^{u}\left(\xi_{1}\right)$. But then $\delta_{1}=\xi_{1}$. But this is also impossible, as seen before. This proves that case II.2 cannot happen either.

Conversely if the $L_{i}, i \in \mathbf{N}$, are eventually in region (4), then we would start with $\alpha \subset$ region (3). The above proof shows there is no chain of lozenges from region (4) to region (3), consequently no chain of lozenges from region (3) to region (4). Therefore this cannot happen either. Finally the symmetry between regions (4) and (5) shows that the sequence $L_{i}, i \in \mathbf{N}$, cannot be eventually in region (5) either.

Since none of the possibilities can occur, we conclude that $\widetilde{\mathcal{F}}^{s}$ has Hausdorff leaf space. It now follows that $\Phi$ is a (topological) $\mathbf{R}$-covered Anosov flow. This finishes the proof of the main theorem.

\section{R-COvered Anosov Flows}

The main theorem leaves open the question as to whether $\mathbf{R}$-covered Anosov flows transverse to $\mathbf{R}$-covered foliations can be non-regulating. At first it may seem this is not possible, but in fact there are many examples.

We first describe the topological types of $\mathbf{R}$-covered Anosov flows. If $\Phi$ is an $\mathbf{R}$-covered Anosov flow, then up to isotopy there are only two topological models for the joint structure of the stable and unstable foliations in the universal cover $\mathrm{Ba2}, \mathrm{Fe3}$, which we now describe.

The set $\mathcal{O}$ is homeomorphic to $H=(0,1) \times \mathbf{R}$, which is more convenient for our description of $\mathbf{R}$-covered Anosov flows. In the product type the foliation $\widetilde{\mathcal{F}}^{s}$ is a foliation by horizontal segments in $H$ and $\widetilde{\mathcal{F}}^{u}$ is a foliation by vertical lines. Notice that any leaf $F$ of $\widetilde{\mathcal{F}}^{s}$ intersects every leaf of $\widetilde{\mathcal{F}}^{u}$ and vice versa.

In the skewed model $\widetilde{\mathcal{F}}^{s}$ is again a foliation by horizontal segments in $H$ and now $\widetilde{\mathcal{F}}^{u}$ is a foliation by parallel segments in $H$ making an angle $\theta \neq \pi / 2$ with the horizontal. Hence any leaf of $\widetilde{\mathcal{F}}^{s}$ does not intersect all leaves of $\widetilde{\mathcal{F}}^{u}$ and vice versa.

For the remainder of this section we consider smooth Anosov flows. In this case there are 1-dimensional strong stable and unstable foliations $\mathcal{F}^{s s}, \mathcal{F}^{u u}$ which are transverse to each other and generated by subbundles $E^{s}, E^{u}$ of the tangent bundle. For details see $[\mathrm{An}, \mathrm{An}-\mathrm{Si}$. 
(a)

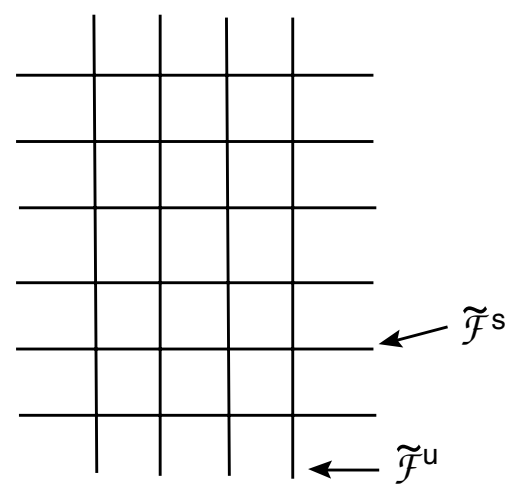

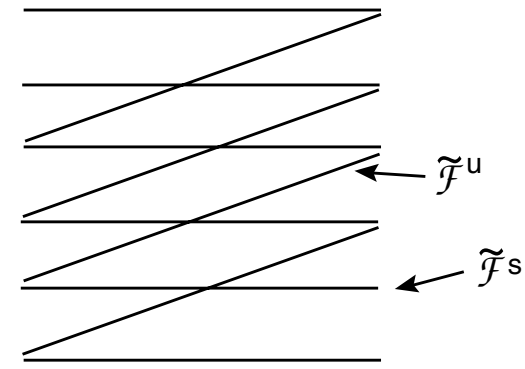

(b)

Figure 11. (a) Product type. (b) Skewed type. The diagrams are bounded in the horizontal direction and unbounded in the vertical direction.

We first study suspension Anosov flows. Let $\mathcal{F}$ be the unstable foliation of a suspension Anosov flow. Then $\mathcal{F}$ is $\mathbf{R}$-covered. Notice that any stable leaf of $\widetilde{\mathcal{F}}^{s}$ intersects every unstable leaf of $\widetilde{\mathcal{F}}^{u}$ in a single orbit of $\widetilde{\Phi}$ and vice versa. We will produce a new Anosov flow, transverse to $\mathcal{F}$ and which is not regulating for $\mathcal{F}$.

Assume that $\mathcal{F}$ is transversely oriented or that $\mathcal{F}^{s s}$ is oriented. For simplicity assume that $M$ has the solv metric so that $T \Phi$ is perpendicular to both the stable $E^{s}$ and unstable $E^{u}$ bundles of $\Phi$. Let $-\pi / 2<\theta<\pi / 2$. Define a new flow $\Phi_{\theta}$ as follows. In each point of $M$ consider the stable leaf $R$ of $\mathcal{F}^{s}$ through it. Staying in $R$, tilt the vector $T \Phi$ by the angle $\theta$; see Figure 12 (a). This can be done coherently because $\mathcal{F}^{s s}$ is oriented. This produces a new vector field $\Phi_{\theta}$. For small $|\theta|$, the vector fields $\Phi_{\theta}$ and $\Phi$ are $C^{1}$-close, so by structural stability of $\Phi$ [An, An-Si], it follows that $\Phi_{\theta}$ is Anosov and topologically conjugate to $\Phi$.

But in fact this is true for any $-\pi / 2<\theta<\pi / 2$ : Since orbits of $\Phi_{\theta}$ are tangent to $\mathcal{F}^{s}$, it follows that the new orbits are contained in the old stable leaves. These flow lines make constant angle $\theta$ with the old flow lines. The stable leaves of $\widetilde{\mathcal{F}}^{s}$ are isometric to the hyperbolic plane $\mathbf{H}^{2}$. Fix a stable leaf $L \in \widetilde{\mathcal{F}}^{s}$. It is easier to see in the upper half plane model for $\mathbf{H}^{2}$ where flow lines of $\widetilde{\Phi}$ are vertical rays and strong stable leaves of $\widetilde{\Phi}$ are horizontal lines (or horocycles); see Figure [12(a). The vertical rays are the intersection of unstable leaves of $\widetilde{\mathcal{F}}^{u}$ with $L$ or the intersections of leaves of $\widetilde{\mathcal{F}}$ with $L$. A curve making a constant angle with the verticals is a ray (Euclidean ray) making angle $\theta$ with vertical direction. This curve is a bounded distance from a vertical line. Still flow lines of $\widetilde{\Phi}_{\theta}$ converge exponentially in forward time. The reader may check that the horizontal vectors are uniformly exponentially contracted in the forward direction. The old strong unstable vectors are still uniformly exponentially expanded in positive time. We conclude that the flow $\Phi_{\theta}$ is Anosov with the same strong stable foliations as those of $\Phi$, same stable foliation, but different unstable foliation.

Notice that for $\Theta=-\pi / 2, \pi / 2$ the flow lines will be tangent to the fibers of a fibration of $M$ over the the circle and the flow $\Phi_{\theta}$ obviously will not be Anosov. 


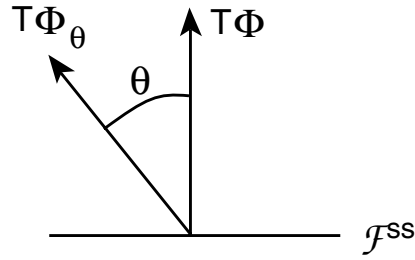

(a)

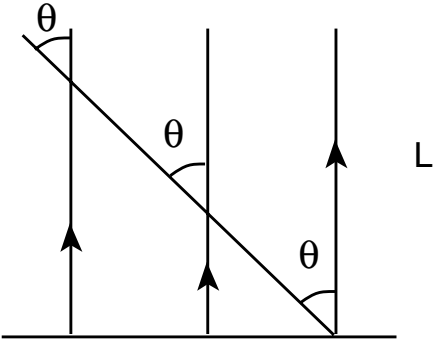

(b)

Figure 12. (a) The tilt of an Anosov flow. (b) The picture in a stable leaf.

For $-\pi<\theta<-\pi / 2$ or $\pi / 2<\theta<\pi$, the flow is conjugate to the inverse of the old one.

Now given an orbit $\gamma$ of $\widetilde{\Phi_{\theta}}$ contained in $L, \gamma$ is a ray making angle $\theta$ with the verticals and $\gamma$ will only intersect a sub-collection of the vertical lines in $L$. Therefore $\gamma$ will not intersect all leaves of $\widetilde{\mathcal{F}}$. We conclude that $\Phi_{\theta}$ is transverse to $\mathcal{F}$ but is not regulating for $\mathcal{F}$ and still $\mathcal{F}$ is $\mathbf{R}$-covered. This provides counterexamples to the main theorem if one allows $\Phi$ to be an $\mathbf{R}$-covered Anosov flow.

Once this example is well understood, it is easy to see that any perturbation of $\Phi$ keeping it tangent to leaves of $\mathcal{F}^{s}$, tranverse to $\mathcal{F}^{u}=\mathcal{F}$ and perturbed with angle $<\pi / 2$, will produce an Anosov flow transverse to $\mathcal{F}$ and which is not regulating for $\mathcal{F}$. We can also do the same for small transverse perturbations of $\Phi$ outside of being tangent to $\mathcal{F}^{s}$.

As for the skewed case: Assume again that $\mathcal{F}^{u}$ is transversely orientable. A small perturbation of $\Phi$, tangent to $\mathcal{F}^{s}$ and transverse to $\mathcal{F}^{u}=\mathcal{F}$, will produce a new flow $\Phi^{\prime}$ topologically conjugate to $\Phi$, hence Anosov. Let $L$ be a leaf of $\widetilde{\mathcal{F}}^{s}$ (same for $\widetilde{\Phi}$ and $\widetilde{\Phi}^{\prime}$ ). Orbits of $\Phi^{\prime}$ in $L$ can only intersect the leaves of $\widetilde{\mathcal{F}}=\widetilde{\mathcal{F}}^{u}$ (unstable from the original flow $\widetilde{\Phi}$ ) intersected by $L$. But these are not all of the leaves of $\widetilde{\mathcal{F}}$ because of the skewed picture. This shows that $\Phi^{\prime}$ is not regulating for $\mathcal{F}$. The easier picture to see in this case is that of a geodesic flow in the unit tangent bundle $T_{1} S, S$ a closed surface of constant negative curvature.

\section{Non-REgulating PSEUdo-Anosov flows AND NON-R-COVERED FOLIATIONS}

In this section we use the main theorem to analyse a large class of known examples of foliations transverse to pseudo-Anosov flows and show that the foliations are not $\mathbf{R}$-covered. There are two types of constructions: branched covers and cut-and-paste operations. This will prove theorem B of the introduction.

I. Branched cover construction - Theorem B, part 1 -

As in the last section consider $\mathcal{F}$ to be the unstable foliation of a suspension Anosov flow $\Phi$. Assume that $\mathcal{F}^{s s}$ is orientable. Fix $\theta \in(-\pi / 2, \pi / 2)$. As in the previous section construct the Anosov flow $\Phi_{\theta}$ which is transverse to $\mathcal{F}$ but not regulating for $\mathcal{F}$. 
Fix a periodic leaf $R$ of $\mathcal{F}^{s}$ with periodic orbit $\gamma_{0}$. Then $\Phi_{\theta}$ also has a periodic orbit $\gamma \subset R$ which is transverse to the flow lines of $\Phi$ when restricted to $R$. The simple closed curve $\gamma$ is transverse to $\mathcal{F}$. Also $\gamma$ is transverse to the fibration of $M$ over the circle associated to the suspension flow $\Phi$.

The complement $M-\gamma$ is irreducible, atoroidal and has an incompressible surface, hence it has a complete hyperbolic metric of finite volume Th2, Th4]. By Thurston's Dehn surgery theorem, for $n$ big enough, the $(0, n)$ Dehn filling along $\gamma$ has a hyperbolic structure [Th2]. Here $(0,1)$ is the meridian, that is, the curve bounding a fiber in $M-\gamma$. Dehn filling produces a hyperbolic orbifold $M_{n}$ with singular locus $\gamma$ and index $n$ along $\gamma$. By Selberg's theorem about finite index subgroups without torsion $\mathrm{Se}$, there is a finite index orbifold cover of $M_{n}$ which is a manifold $N$. In other words $N$ is a branched cover of $M$ with branch locus $\gamma$ and index $n$ along $\gamma$. For background on branched covers consult Rol.

Since the branch set is a closed orbit $\gamma$ of $\Phi_{\theta}$, the branched cover construction produces a flow $\Phi_{1}$ in $N$ which is a branched cover of the flow $\Phi_{\theta}$. Locally near $\gamma$ the construction unwraps the manifold $M_{n} n$ times around $\gamma$, therefore the new flow $\Phi_{1}$ is a pseudo-Anosov flow in $N$. The only singular orbits are those coming from $\gamma$, where there is an $n$-to- 1 branched cover of a neighborhood of $\gamma$.

The curve $\gamma$ is transverse to $\mathcal{F}$ in $M$, hence leaves of $\mathcal{F}$ meet a neighborhood of $\gamma$ in meridian disks. Under the branched cover construction $N \rightarrow M_{n}, \mathcal{F}$ lifts to a foliation $\mathcal{F}_{1}$ in $N$. Locally near lifts of $\gamma$, leaves of $\mathcal{F}_{1}$ are $n$-to- 1 branched covers of disks in leaves of $\mathcal{F}$. The pseudo-Anosov flow $\Phi_{1}$ in $N$ is transverse to the foliation $\mathcal{F}_{1}$. The foliation $\mathcal{F}_{1}$ is the one we are interested in analyzing.

We claim that $\Phi_{1}$ is not regulating for $\mathcal{F}_{1}$. To see this consider first the universal cover $\widetilde{M}$ of $M$, with lifts $\widetilde{\mathcal{F}}, \widetilde{\Phi}$. Let $Z$ be the leaf of $\mathcal{F}$ containing $\gamma_{0}$, that is, the unstable leaf (of $\Phi$ !) containing $\gamma_{0}$. Let $\widetilde{\gamma}$ be a lift of $\gamma$ to $\widetilde{M}$ and $F \in \widetilde{\mathcal{F}}$ the corresponding lift of $Z$. By construction of $\gamma$ and $Z$, it follows that $\widetilde{\gamma} \cap F=\emptyset$. The universal cover $\widetilde{N}$ is a branched cover of $\widetilde{M}$, branched along all lifts of $\gamma$ to $\widetilde{M}$. It follows that any lift of $\widetilde{\gamma}$ to $\widetilde{N}$ will not intersect any lift of $F$ to $\widetilde{N}$. Hence some orbits of $\widetilde{\Phi}_{1}$ do not intersect every leaf of $\widetilde{\mathcal{F}}_{1}$. It follows that $\Phi_{1}$ is not regulating for $\mathcal{F}_{1}$.

Since $\Phi_{1}$ is obviously a singular pseudo-Anosov flow, it cannot be an $\mathbf{R}$-covered Anosov flow. If $\mathcal{F}_{1}$ were $\mathbf{R}$-covered, the flow $\Phi_{1}$ would have to be regulating, by the main theorem. We conclude that $\mathcal{F}_{1}$ is not $\mathbf{R}$-covered.

The orbit $\gamma$ of $\Phi_{\theta}$ is transverse to the original fibration of $M$ over the circle. Under the branched cover construction, the fibration in $M$ lifts to a fibration in $N$ and hence $\Phi_{1}$ is a suspension pseudo-Anosov flow.

In summary the main ingredient for the construction to work is a branch cover along a closed orbit of the given flow which is not regulating for the original foliation. It follows that this applies to a large class of examples.

II. Cut and paste construction - Theorem B, part 2 -

The second class of examples is obtained by cut-and-paste operations on a fibration. Let $M$ be a manifold fibering over the circle with pseudo-Anosov monodromy $\eta$, that is, $M=S \times[0,1] /(x, 1) \cong(\eta(x), 0)$, where $S$ is a hyperbolic surface and $\eta$ is a pseudo-Anosov homeomorphism of $S$ Th3, FLP. Suppose that all singularities of $\eta$ have an even number of prongs. This can be achieved for instance by taking branched covers of Anosov diffeomorphisms of the torus $T^{2}$; see expose 13 in [FLP]. 


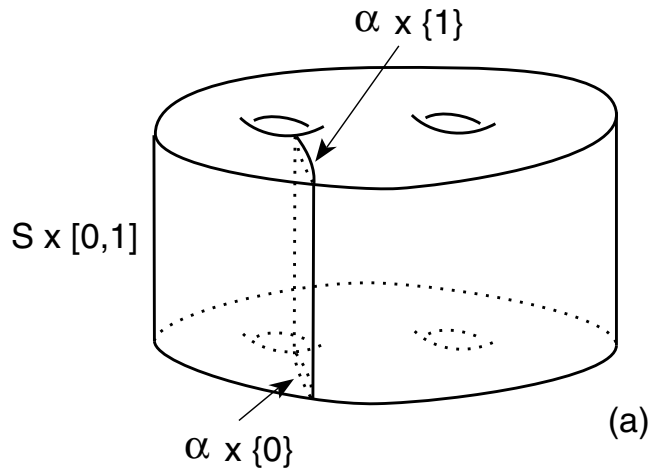

(a)

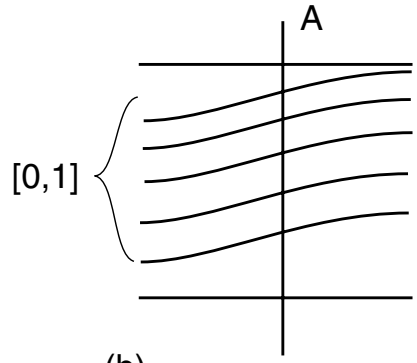

(b)

Figure 13. (a) Cutting and pasting in fibrations. (b) Shearing along an annulus.

Then choose $\eta$ so that there is a simple closed curve $\alpha$ in $S$ with $\alpha \cap \eta(\alpha)=\emptyset$. This can also be achieved by taking finite covers and the fact that $\pi_{1}(S)$ is LERF $[\mathrm{Sc}$ (see explicit construction in section 2 of [CLR1]). Let $\lambda$ be the expansion/contraction constant associated to the homeomorphism $\eta$, that is, under $\eta$ unstable lengths get multiplied by $\lambda$ and stable lengths get divided by $\lambda$.

Now consider the suspension flow $\Phi$ of $\eta$ which is a suspension pseudo-Anosov flow. This flow will be fixed throughout the discussion. Let $\mathcal{F}_{0}$ be the original fibration of $M$.

In CLR1 Cooper, Long and Reid did cut and paste to the fiber $S$ along an annulus transverse to the fibration and with base in $\alpha \subset S$ to obtain closed immersed surfaces transverse to $\Phi$. Many of these surfaces were not virtual fibers producing fascinating examples. Here we do cut and paste to produce a non-R-covered foliation.

Let $A$ be the annulus $\alpha \times[0,1]$ in $S \times[0,1]$. Since $\alpha \cap \eta(\alpha)=\emptyset$, then $A$ embeds in $M$. Extend $A$ a little bit beyond both boundaries to get an embedded annulus in $M$ transverse to $\mathcal{F}_{0}$ with boundaries in leaves of $\mathcal{F}_{0}$ covering a little more than one period of the fibration. Let this be the annulus $A$ thought to be in $M$. Then $\mathcal{F}_{0}$ induces a foliation by circles on $A$ - parametrize this as $\alpha \times[-a, 1+a]$ (where $a>0$ ). Cut open $M$ along $A$ and glue back by $\alpha \times\{t\} \rightarrow \alpha \times\{h(t)\}$, where $h$ is a homeomorphism of $[-a, 1+a]$ so that $h(x)>x$ for any $x \in(-a, 1+a)$. There is $b>0$ so that for any $x \in[0,1]$, then $h(x)>x+b$. The cut and paste of $\mathcal{F}_{0}$ produces a new foliation $\mathcal{F}$ still transverse to the suspension flow $\Phi$.

This construction is well known to Thurston, Gabai and others. Here we analyse the leaf space of the ensuing foliations.

We think of $\Phi$ as a true suspension so that it takes time 1 to go between any two lifts of $S$ to $\widetilde{M}$. We will measure height in $\widetilde{M}$ using the time parameter. Let $\widetilde{S}_{0}$ be the lift of $S$ at height 0 .

Think of $S$ as $S \times\{0\}$ embedded in $M$. Let $\mathcal{F}_{0}^{u u}$ be the singular strong unstable folation of $\eta$ in $S$. Notice $\mathcal{F}_{0}^{u u}$ is orientable because of the even parity of prongs. We can choose $\alpha$ so that it is transverse to $\mathcal{F}_{0}^{u u}$. Therefore following leaves of $\mathcal{F}_{0}^{u u}$ in the positive direction, they will always intersect $\alpha$ in the same direction, which 
we may assume is the direction which increases the height under the gluing by $h$. In addition, since leaves of $\mathcal{F}_{0}^{u u}$ are dense in $S$, any segment in a leaf of $\mathcal{F}_{0}^{u u}$ intersects $\alpha$ after at most length $c_{0}>0$. It easily follows that there is $c>0$ so that any segment of a leaf of $\mathcal{F}^{u u}$ (of $\Phi$ !) of length $>c$ has to intersect $\alpha \times[0,1]$.

Let $F$ be a leaf of $\widetilde{\mathcal{F}}$. Since $\widetilde{\Phi}$ is transverse to $F$, the unstable foliation $\widetilde{\mathcal{F}}^{u}$ induces a singular one-dimensional foliation $\widetilde{\mathcal{F}}_{F}^{u}$ in $F$. Let $l$ be a leaf of $\widetilde{\mathcal{F}}_{F}^{u}$, contained in $L \in \widetilde{\mathcal{F}}^{u}$. We will keep track of the progress of this leaf in $\widetilde{M}$. For simplicity suppose we start at a point in $l$ of height 0 and follow $l$ in the positive direction. Assume $L$ is non-singular. Each time $l$ crosses a lift of $\alpha \times[0,1]$, then $l$ goes up by at least height $b$ and this happens at least every length $c$ in $l$. Projecting back to $\widetilde{S}_{0}$ along flow lines of $\widetilde{\Phi}$ the distances along $l$ are contracted by at least $\lambda^{b}$ as compared to what happened before the crossing [Ca-Th]. Therefore as seen from the projection in $\widetilde{S}$, then after length at most $\lambda^{-b} c$, the leaf $l$ will hit a lift of $\alpha \times[0,1]$ again and $l$ goes up again by at least height $b$. When projecting to $\widetilde{S}$ distance are now contracted by at least $\lambda^{2 b}$. By induction after length at most $k c$ along $l$, which corresponds to the length

$$
c+\lambda^{-b} c+\lambda^{-2 b} c+\ldots+\lambda^{-b(k-1)} c=c \frac{1-\lambda^{-b k}}{1-\lambda^{-b}}
$$

when projected to $\widetilde{S}$, then $l$ is at a height at least $k b$. Let $l^{\prime}$ be $L \cap \widetilde{S}_{0}$. By letting $k \rightarrow \infty$ in the argument above we see that the height of $l$ blows up to $\infty$ before the projection of $l$ to $l^{\prime}$ achieves a length of

$$
c \frac{1}{1-\lambda^{-b}}
$$

from the initial point in $l^{\prime}$. It follows that $F$ cannot intersect the orbit of $\widetilde{\Phi}$ corresponding to the intersection of $\widetilde{S}$ with the limit of the projection of $l$ to $l^{\prime}$. Otherwise by uniqueness of intersections of $F$ with orbits of $\widetilde{\Phi}$, the height of $l$ would be bounded near this limit orbit, contradiction to it increasing without bound.

We conclude that $F$ does not intersect this orbit of $\widetilde{\Phi}$ and hence $\Phi$ is not regulating for $\mathcal{F}$. Since $\Phi$ is a singular pseudo-Anosov flow the main theorem implies that $\mathcal{F}$ is not $\mathbf{R}$-covered. This finishes the analysis of the second class of examples.

\section{Rigidity of foliations transverse to R-COVERED Anosov flows}

If $\Phi$ is an Anosov flow transverse to an $\mathbf{R}$-covered foliation $\mathcal{F}$, then as seen in section [5, $\Phi$ may fail to be regulating if $\Phi$ is an $\mathbf{R}$-covered Anosov flow. We will show in this section that this failure is very rare. The goal is to prove a topological rigidity result: if needed, blow down some foliated $I$-bundles of $\mathcal{F}$, then the resulting foliation is topologically conjugate to one of the invariant foliations of $\Phi$.

First we need some facts about the structure of $\mathbf{R}$-covered Anosov flows. Consider $\Phi$ an $\mathbf{R}$-covered Anosov flow of product type. If $g$ is a covering translation of $\widetilde{M}$, then $g$ leaves invariant at most one leaf of $\widetilde{\mathcal{F}}^{s}$ (or $\widetilde{\mathcal{F}}^{u}$ ) [Fe3]. Equivalently there are no non-trivial free homotopies between closed orbits of $\Phi$.

In this case $\pi_{1}(M)$ is solvable and there is important work of Plante [P12] completely classifying foliations in such manifolds. However Plante assumes that $\mathcal{F}$ is at least $C^{2}$ and he makes use of Kopell's lemma [Ko. We do not assume that $\mathcal{F}$ is $C^{2}$, in fact in this particular case it is very easy to produce $\mathcal{F}$ transverse to $\Phi$ which cannot be $C^{2}$. 


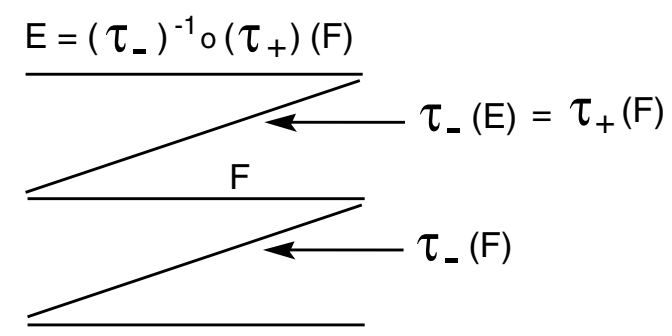

Figure 14. The skewed picture produces a slithering over the circle.

Recall that $\mathcal{H}$ is the leaf space of $\widetilde{\mathcal{F}}$ and similarly $\mathcal{H}^{s}, \mathcal{H}^{u}$.

The structure of a skewed $\mathbf{R}$-covered pseudo-Anosov flow is much more complex and rich. Let $\Phi$ have skewed type. For simplicity suppose that $\mathcal{F}^{s}, \mathcal{F}^{u}$ are transversely orientable. Fix a transverse orientation to $\mathcal{F}^{s}$ and put an order $<_{s}$ in $\mathcal{H}^{s}$ coherent with this transverse orientation. Given $F \in \widetilde{\mathcal{F}}^{s}$ there is a unique unstable leaf $U$ which makes a perfect fit with $F$ and is contained in the component of $\widetilde{M}-F$ defining the positive orientation from $F$ Fe3. Intuitively the leaf $U$ is "asymptotic" to $F$; see Figure 14. Let this $U$ be denoted by $\tau_{+}(F)$. Then $\tau_{+}$ defines a homeomorphism from $\mathcal{H}^{s}$ to $\mathcal{H}^{u}$. Similarly define $\tau_{-}(F)$ which makes a perfect fit with $F$ and is on the negative side of $F$; see Figure 14. Then $\tau_{-}$is also a homeomorphism from $\mathcal{H}^{s}$ to $\mathcal{H}^{u}$. We choose a transversal orientation $<_{u}$ to $\mathcal{F}^{u}$ so that $\tau_{-}(F)<_{u} \tau_{+}(F)$.

Let $\zeta: \mathcal{H}^{s} \rightarrow \mathcal{H}^{s}$ be the homeomorphism $\left(\tau_{-}\right)^{-1} \circ \tau_{+}$. Then $\zeta$ is fixed point free [Fe3, Fe6]. Intuitively it goes one step up in the infinite ladder of asymptotic leaves. Then $\mathcal{H}^{s} / \zeta$ is homeomorphic to a circle and there is a map $\delta: \widetilde{M} \rightarrow \mathbf{S}^{1}$ induced by the map $\mathcal{H}^{s} \rightarrow \mathcal{H}^{s} / \zeta$. The map $\delta$ is group equivariant: there is a group homomorphism $\rho: \pi_{1}(M) \rightarrow H o m e o\left(\mathbf{S}^{1}\right)$, so that

$$
\forall g \in \pi_{1}(M), \quad \forall x \in \widetilde{M}, \quad \delta(g(x))=\rho(g)(\delta(x)),
$$

or $\delta \circ g=\rho(g) \circ \delta\left[\mathrm{Fe} 3\right.$, Fe6]. Therefore the foliation $\widetilde{\mathcal{F}}^{s}$ (and consequently $\mathcal{F}^{s}$ ) is induced by $\delta$. The map $\delta$ is an example of what Thurston calls a slithering over the circle Th5]. We refer the reader to Th5] for the beautiful theory of slitherings. They are very common in 3-manifolds. We will use a few simple properties of foliations defined by slitherings. One property which follows easily from the slithering map is that given any $F, L \in \widetilde{\mathcal{F}}^{s}$ they are a bounded (Hausdorff) distance apart: there is $c_{0}>0$ so that for any $x \in F, y \in L$ then $d(x, L)<c_{0}$ and $d(y, F)<c_{0}$. Here the number $d(x, L)$ is the infimum of $d(x, z)$ for all $z \in L$. Clearly $c_{0}$ depends on the pair $F, L$. Let $d_{H}(F, L)$ be the smallest $c_{0}$ which works. This is the Hausdorff distance between leaves in $\widetilde{M}$.

Notice that distance in $\mathcal{H}^{s}$ is not well defined but slithering units are: we say that $a, b \in \mathcal{H}^{s}$ are $n$ slithering units apart if $a=\zeta^{n}(b)$ or $b=\zeta^{n}(a)$. Then given any $a, b \in \mathcal{H}^{s}$ with $a<_{s} b$ let

$$
d_{s}(a, b)=n, \text { if } \zeta^{n}(a) \leq_{s} b<_{s} \zeta^{n+1}(a) .
$$

Extend it to $a \geq_{s} b$ by making $d_{s}$ symmetric. This is a roughly-defined distance in $\mathcal{H}^{s}$. One fundamental fact is that $d_{s}$ is invariant under covering translations: if 
$g \in \pi_{1}(M)$, then for any $a, b \in \mathcal{H}^{s}, d_{s}(a, b)=d_{s}(g(a), g(b))$. This follows from the invariance of the infinite staircase picture. Another important fact proved in Th5] is that there are constants $c_{1}, c_{2}$ so that for any $E, L \in \widetilde{\mathcal{F}}^{s}$ then

$$
c_{1}\left(d_{s}(E, L)-1\right) \leq d_{H}(E, L) \leq c_{2}\left(d_{s}(E, L)+1\right) .
$$

This says that the slithering distance between leaves roughly measures the Hausdorff distance between leaves. In addition the distance between points in leaves is also bounded below: there is $c_{3}>0$ so that for any $E, L \in \widetilde{\mathcal{F}}^{s}$ then

$$
\forall p \in E, q \in L, \quad d(p, q)>c_{3}\left(d_{s}(E, L)-1\right) .
$$

Whenever the distance $d_{s}$ is used, it is implicit that $\Phi$ is a skewed $\mathbf{R}$-covered Anosov flow and $d_{s}$ refers to the distance defined here.

Many 3-manifolds containing skewed Anosov flows are Seifert fibered. Ghys Gh showed the manifold is up to finite covers $T_{1} S$, where $S$ is a hyperbolic surface. Foliations in Seifert fibered manifolds have also been extensively studied by Thurston Th1], Levitt [Le and finally Brittenham $\mathrm{Br}$. This could help us a bit in the Seifert fibered case. However, skewed R-covered Anosov flows can occur in much more general manifolds, for instance the manifolds may be hyperbolic [ $\mathrm{Fe} 3$.

Unless otherwise stated, for the remainder of this section $\Phi$ will denote an Rcovered Anosov flow and $\mathcal{F}$ a transverse foliation. We first show that $\mathcal{F}$ has to be R-covered.

Proposition 7.1. If $\Phi$ is an $\mathbf{R}$-covered Anosov flow transverse to a foliation $\mathcal{F}$, then $\mathcal{F}$ is an $\mathbf{R}$-covered foliation.

Proof. Let the positive transversal direction to $\mathcal{F}$ be defined by positive flow direction of $\Phi$. Up to a double cover if necessary, assume that $\mathcal{F}^{s}$ is transversely oriented. We will later see that this hypothesis is not necessary. In this proof we assume that the order $<_{s}$ in $\mathcal{H}^{s}$ is coherent with the transverse orientation to $\mathcal{F}^{s}$. Notice that it is preserved by covering translations.

Suppose the proposition is not true. Clearly $\Phi$ is not regulating for $\mathcal{F}$. Let $F_{0}, F_{1} \in \widetilde{\mathcal{F}}$ not be separated from each other, say in their negative sides. As they are not separated, they cannot intersect a common orbit of $\widetilde{\Phi}$, so $\Theta\left(F_{0}\right) \cap \Theta\left(F_{1}\right)=\emptyset$. Hence there is either a stable or unstable boundary leaf of $\Theta\left(F_{0}\right)$ separating it from $\Theta\left(F_{1}\right)$. If it is unstable, then the corresponding unstable leaf of $\widetilde{\mathcal{F}}^{u}$ is on the positive side of $F_{0}$. Therefore $F_{1}$ is also in the positive side of $F_{0}$, a contradiction to the assumption. Let therefore $L_{0}$ be a leaf of $\widetilde{\mathcal{F}}^{s}$ with $\Theta\left(L_{0}\right) \subset \partial \Theta\left(F_{0}\right)$ and $L_{0}$ separating $F_{0}$ from $F_{1}$. In the same way there is $L_{1} \in \tilde{\mathcal{F}}^{s}$ with $\Theta\left(L_{1}\right) \subset \partial \Theta\left(F_{1}\right)$ and $L_{1}$ separating $F_{1}$ from $F_{0}$. Notice that $L_{0}$ and $L_{1}$ may be the same leaf. Let $l_{i}=\Theta\left(L_{i}\right)$. Assume that $F_{1}$ intersects stable leaves which are $<_{s} L_{0}$; see Figure 15(a). Given these conditions we make the following claim.

Claim - For any leaf $F \in \widetilde{\mathcal{F}}$, then $\partial \Theta(F)$ is a union of two stable leaves of $\widetilde{\mathcal{F}}_{\mathcal{O}}^{s}$.

We first prove the claim for $F_{0}$ defined above. If the boundary of $\Theta\left(F_{0}\right)$ consists only of $l_{0}$, then $F_{0}$ intersects all $L \in \widetilde{\mathcal{F}}^{s}$ with $L>_{s} L_{0}$. Barbot Ba2] showed that $\Phi$ is transitive, hence $\pi\left(L_{0}\right)$ is dense in $M$. Take a covering translate $g\left(L_{0}\right)$ of $L_{0}$ so that $g\left(L_{0}\right) \cap F_{1} \neq \emptyset$. Hence $g\left(L_{0}\right)<_{s} L_{1}$. We now consider the picture near $g\left(L_{0}\right)$. Let $p \in \Theta\left(g\left(L_{0}\right)\right) \cap \Theta\left(F_{1}\right)$ and $p_{i} \in \Theta\left(g\left(F_{0}\right)\right)$ with $p_{i} \rightarrow p$. Let $y_{i} \in F_{1}$ with $\Theta\left(y_{i}\right)=p_{i}$. Since $p \in \Theta\left(F_{1}\right)$ then $y_{i} \rightarrow y$ with $\Theta(y)=p$. Let $x \in g\left(F_{0}\right)$ with $\Theta\left(x_{i}\right)=p_{i}$. As $p \in \partial \Theta\left(g\left(F_{0}\right)\right)$, then $x_{i}=\widetilde{\Phi}_{t_{i}}\left(y_{i}\right)$ with $\left|t_{i}\right| \rightarrow \infty$. Since $\Theta\left(g\left(L_{0}\right)\right)$ 


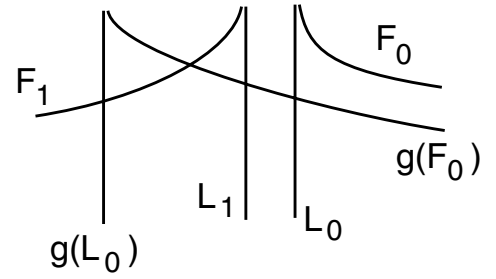

(a)

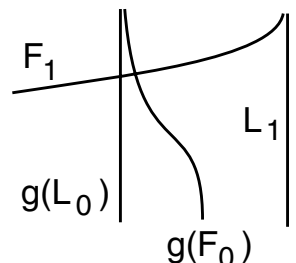

(b)

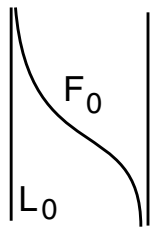

$g\left(F_{0}\right)$

Figure 15. (a) The case where boundary of $\partial \Theta\left(F_{0}\right)$ has only one component produces impossible intersection. (b) An unstable boundary also produces impossible intersection.

is a stable boundary leaf of $\Theta\left(g\left(F_{0}\right)\right)$ it follows that $t_{i} \rightarrow+\infty$. This implies that $g\left(F_{0}\right)$ is in the front of $F_{1}$. Applying the same argument to points in $L_{1}$ we obtain that $g\left(F_{0}\right)$ is in the back of $F_{1}$. But that would imply that $g\left(F_{0}\right)$ and $F_{1}$ have to intersect, which is a contradiction; see Figure 15(a).

Hence $\Theta\left(F_{0}\right)$ has more boundary. If it is unstable boundary, then points in $F_{0}$ go down when points in $\Theta\left(F_{0}\right)$ approach this boundary. Take $g\left(L_{0}\right) \cap F_{1} \neq \emptyset$. As seen in the argument of the previous paragraph this implies the contradiction $g\left(F_{0}\right) \cap F_{1} \neq \emptyset$; see Figure 15(b). We conclude that there should be more stable boundary to $\Theta\left(F_{0}\right)$. Finally given any 3 stable leaves of $\widetilde{\mathcal{F}}^{s}$, one of them separates the other two, hence it follows that $\partial \Theta\left(F_{0}\right)$ can have only two components. This proves the claim for $F_{0}$.

Let $l_{0}^{\prime}$ be the other component of $\partial \Theta\left(F_{0}\right)$ and let $L_{0}^{\prime} \in \widetilde{\mathcal{F}}^{s}$ with $\Theta\left(L_{0}^{\prime}\right)=l_{0}^{\prime}$. Now given any $F \in \widetilde{\mathcal{F}}$, there is $h(F)$ which is in front of $F_{0}$. But then $h(F)$ cannot intersect $L_{0}$ or $L_{0}^{\prime}$. Therefore $h(F)$ has to escape in the positive direction before it gets to $L_{0}$ or $L_{0}^{\prime}$. It follows that $\partial \Theta(h(F))$ also has two components and the same holds for $F$. This finishes the proof of the claim.

It is this claim that will eventually lead to a contradiction.

If all leaves of $\mathcal{F}$ are simply connected, then $\mathcal{F}$ is a foliation by planes and this implies that $M$ is the 3 -torus $T^{3}\left[\operatorname{Ros}\right.$. But $T^{3}$ does not support an $\mathbf{R}$-covered Anosov flow, because it has abelian fundamental group and 3-manifolds supporting R-covered Anosov flows have exponential fundamental group [Pl-Th].

Then let $F_{2}$ be a leaf with $\pi\left(F_{2}\right)$ not simply connected. Let $g \in \pi_{1}(M)$, not the identity, with $g\left(F_{2}\right)=F_{2}$. Let $\partial \Theta\left(F_{2}\right)=l_{2} \cup l_{2}^{\prime}$ and $L_{2}, L_{2}^{\prime} \in \tilde{\mathcal{F}}^{s}$ with $\Theta\left(L_{2}\right)=l_{2}$, $\Theta\left(L_{2}^{\prime}\right)=l_{2}^{\prime}$. Since $g$ preserves orientation of $\mathcal{H}^{s}$, the fact that $g\left(F_{2}\right)=F_{2}$ implies that $g\left(L_{2}\right)=L_{2}$ and $g\left(L_{2}^{\prime}\right)=L_{2}^{\prime}$.

If $\Phi$ has product type this immediately leads to a contradiction, because $g$ would have two invariant leaves in $\widetilde{\mathcal{F}}^{s}$.

In the skewed case this does not yet lead to a contradiction. Suppose that $d_{s}\left(L_{2}, L_{2}^{\prime}\right)=n$. Consider $h \in \pi_{1}(M)$, with $h\left(L_{2}\right)>_{s} L_{2}^{\prime}$ and $d_{s}\left(L_{2}, h\left(L_{2}\right)\right)>n+3$. Fix an orbit $\gamma$ in $L_{2}$. Consider $E \in \widetilde{\mathcal{F}}$ with $E \cap L_{2} \neq \emptyset$. Then $E \cap \gamma \neq \emptyset$. Suppose first that no such $E$ intersects $h\left(L_{2}\right)$. Let $E_{i}$ be a sequence of leaves of $\widetilde{\mathcal{F}}^{s}$ so that $E_{i} \cap \gamma$ is monotonic in $\gamma$ and escapes in the negative flow direction of $\gamma$. Let $S_{i}$ be the stable leaf with $S_{i}>{ }_{s} L_{2}$ and $\Theta\left(S_{i}\right) \subset \partial \Theta\left(E_{i}\right)$; see Figure 16. Because $E_{i} \cap \gamma$ is monotonic in $\gamma$ then $S_{i}$ are (weakly) monotonic and increasing in $\mathcal{H}^{s}$. But since 


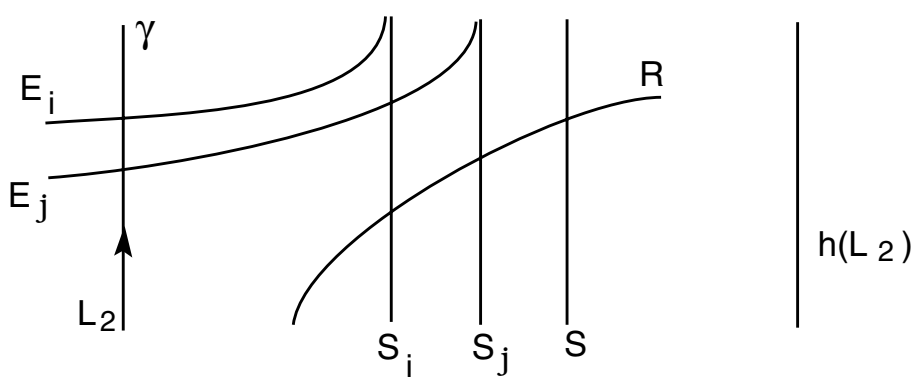

Figure 16. Producing unstable boundary.

no $E_{i}$ intersects $h\left(L_{2}\right)$ then $S_{i} \leq_{s} h\left(L_{2}\right)$. Therefore $S_{i} \rightarrow S$ in $\mathcal{H}^{s}$ as $i \rightarrow \infty$; see Figure 16.

Choose $R$ in $\mathcal{F}$ with $R \cap S \neq \emptyset$. Then $R$ is in the back of any $E_{i}$ so $R \cap L_{2}=\emptyset$. Also $R \cap S_{i} \neq \emptyset$ for $i$ big enough and therefore $R$ cannot escape in the positive direction before getting to $L_{2}$. One concludes that $R$ has to escape down, producing unstable boundary in $\Theta(R)$; see Figure 16. This contradicts the claim above.

We conclude that there is $E$ with $E \cap L_{2} \neq \emptyset$ and $E \cap h\left(L_{2}\right) \neq \emptyset$. Let $A_{0}, A_{0}^{\prime} \in \widetilde{\mathcal{F}}^{s}$ with $\partial \Theta(E)=\Theta\left(A_{0}\right) \cup \Theta\left(A_{0}^{\prime}\right)$. Then $A_{0}<_{s} L_{2}$ and $A_{0}^{\prime}>_{s} h\left(L_{2}\right)$. Therefore $d_{s}\left(A_{0}, A_{0}^{\prime}\right) \geq n+3$.

Choose $f \in \pi_{1}(M)$ with $f(E)$ in front of $F$. This implies that

$$
L_{2} \leq_{s} f\left(A_{0}\right)<_{s} f\left(A_{0}^{\prime}\right) \leq_{s} L_{2}^{\prime}
$$

Then

$$
d_{s}\left(f\left(A_{0}\right), f\left(A_{0}^{\prime}\right)\right) \leq d_{s}\left(L_{2}, L_{2}^{\prime}\right)=n \leq d_{s}\left(A_{0}, A_{0}^{\prime}\right)-3
$$

This contradicts the fact that the covering translation $f$ preserves $d_{s}$. This contradiction shows there is also a problem in the skewed case. We conclude that the claim is impossible, that is, for any $F \in \widetilde{\mathcal{F}}^{s}$, the set $\Theta(F)$ can have at most one stable boundary component. Finally, the failure of the claim implies that $\mathcal{F}$ is $\mathbf{R}$-covered as desired. This finishes the proof of proposition 7.1 .

Given that $\mathcal{F}$ is $\mathbf{R}$-covered, we now start to analyse the relationships between $\mathcal{F}, \mathcal{F}^{s}, \mathcal{F}^{u}$. We first lift to a double cover if necessary so that $\mathcal{F}^{s}$ is transversely orientable. As shown by Barbot [Ba2, this implies in the skewed case that $\mathcal{F}^{u}$ is also transversely oriented. The standing hypothesis for the remainder of this section (unless otherwise stated) is that there is a fixed leaf $F^{*} \in \widetilde{\mathcal{F}}$ with $\Theta\left(F^{*}\right)$ having a stable boundary component.

Lemma 7.2. For any $F \in \widetilde{\mathcal{F}}$ the set $\Theta(F)$ has a unique stable boundary component.

Proof. The leaf $F^{*}$ has a stable boundary component $l^{*}$. Since $\Phi$ is transitive, it follows from symbolic dynamics [Bow1, Bow2 that there are dense orbits of $\Phi$ in $M$. Let $\gamma$ be an orbit of $\Phi$ which is dense in $M$ and let $\widetilde{\gamma}$ be a lift of $\gamma$ which intersects $F^{*}$. There is a translate $f_{0}(F)$ of $F$ so that $\widetilde{\gamma}$ intersects $f_{0}(F)$ after intersecting $F^{*}$. Then $\Theta\left(F^{*}\right), \Theta\left(f_{0}(F)\right)$ intersect in a point $p=\Theta(\widetilde{\gamma}) \in \mathcal{O}$. Let $\alpha$ be a path in $\Theta\left(F^{*}\right) \cup l^{*}$ from $p$ to $p^{\prime} \in l^{*}$, intersecting $l^{*}$ only in $p^{\prime}$. This path cannot be totally lifted to $F^{*}$ because it escapes in the positive direction as the projection to 
$\mathcal{O}$ approches $p^{\prime}$. Since $f_{0}(F)$ is above $F^{*}$, the path cannot be totally lifted to $f_{0}(F)$ either and has to escape in the positive flow direction before the projection in $\mathcal{O}$ gets to $p^{\prime}$. This produces stable boundary in $\Theta\left(f_{0}(F)\right)$. Translating back by $f_{0}^{-1}$ implies that $\Theta(F)$ also has stable boundary.

Finally the proof of the previous proposition shows that there is at most one boundary stable leaf.

These results show that given $F \in \widetilde{\mathcal{F}}$ there is a unique $L \in \widetilde{\mathcal{F}}^{s}$ with $\Theta(L) \subset$ $\partial \Theta(F)$. Let this $L$ be $\eta_{s}(F)$. This defines a function $\eta_{s}: \mathcal{H} \rightarrow \mathcal{H}^{s}, \eta_{s}(F)=L$. The function $\eta_{s}$ commutes with covering translations:

$$
\forall g \in \pi_{1}(M), \quad \eta_{s} \circ g=g \circ \eta_{s} .
$$

We now fix a transversal orientation $<_{s}$ to $\mathcal{F}^{s}$ so that under this orientation $F^{*}$ is contained in the front of $\eta_{s}\left(F^{*}\right)$. As explained in the beginning of the section this defines a transversal orientation $<_{u}$ to $\mathcal{F}^{u}$ so that $\tau_{+} \circ\left(\tau_{-}\right)^{-1}$ is increasing in $\mathcal{H}^{u}$.

Recall that $\eta_{s}\left(F^{*}\right)$ is in the back of $F^{*}$ with respect to positive flow direction of $\widetilde{\Phi}$, hence $\eta_{s}\left(F^{*}\right)$ is in the back of $F^{*}$ with respect to the transverse orientation of $\widetilde{\mathcal{F}}$. Given a translate $g\left(F^{*}\right)$ of $F^{*}$ and $L^{*}=\eta_{s}\left(F^{*}\right)$, then $\eta_{s}\left(g\left(F^{*}\right)\right)=g\left(L^{*}\right)$ is in the back of $g\left(F^{*}\right)$ (as seen from the transverse orientation to $\widetilde{\mathcal{F}}$ ) and $g\left(F^{*}\right)$ is in the front of $g\left(L^{*}\right)$ (as seen from the transverse orientation to $\widetilde{\mathcal{F}}^{s}$ ). Therefore because the translates of $L^{*}$ are dense in $\widetilde{M}$, this property follows for all $F \in \widetilde{\mathcal{F}}$ and $L=\eta_{s}(F) \in \widetilde{\mathcal{F}}^{s}$ - this is because $\mathcal{F}$ is $\mathbf{R}$-covered. Hence if $F_{1}<F_{2}$ in $\mathcal{H}$, then $F_{2}$ is in the front of $F_{1}$ and so $F_{1}$ separates $\eta_{s}\left(F_{1}\right)$ from $F_{2}$. It follows that $\eta_{s}\left(F_{2}\right)$ is also in the front of $\eta_{s}\left(F_{1}\right)$ (from the point of view of $\widetilde{\mathcal{F}}^{s}$ ). This implies that $\eta_{s}: \mathcal{H} \rightarrow \mathcal{H}^{s}$ is a weakly monotone increasing map.

If the original $\mathcal{F}^{s}$ is not transversely orientable, there is $g \in \pi_{1}(M)$ reversing any orientation to $\mathcal{H}^{s}$. Then $g\left(F^{*}\right)$ would be in the back of $\eta_{s}\left(g\left(F^{*}\right)\right)$, contradicting the discussion above. It follows that the original $\mathcal{F}^{s}$ is transversely orientable and it is not necessary to lift to a double cover. In addition if $\Phi$ is skewed, then $\mathcal{F}^{u}$ is also transversely oriented.

We stress the fact that both $\mathcal{H}$ and $\mathcal{H}^{s}$ are homeomorphic to the real numbers is essential throughout the proofs in this section. The leaf $F^{*}$ of $\widetilde{\mathcal{F}}$ is fixed throughout the discussion and let $L^{*}=\eta_{s}\left(F^{*}\right)$.

Lemma 7.3. The map $\eta_{s}: \mathcal{H} \rightarrow \mathcal{H}^{s}$ is continuous.

Proof. Consider $F_{i} \in \mathcal{H}$ with $F_{i} \rightarrow F$ in $\mathcal{H}$. We may assume that $F_{i}$ is monotonic in $\mathcal{H}$.

$\underline{\text { Case } 1}-F_{i}$ is decreasing.

Then $L_{i}=\eta_{s}\left(F_{i}\right)$ is weakly monotone decreasing. Clearly $L=\eta_{s}(F) \leq_{s} \eta_{s}\left(F_{i}\right)$ for all $i$, hence $L_{i}$ is bounded below and $L_{i} \rightarrow L^{-}$in $\mathcal{H}^{s}$. If $L<_{s} L^{-}$, then either $F$ intersects $L^{-}$or $L^{-}$separates $L_{i}$ from $F$ for all $i$; see Figure 17(a). Hence for a fixed $j$ big enough, either $F_{j}$ intersects $L^{-}$or $F_{j}$ jumps to the other side of $L^{-}$. Both options are disallowed by $L_{i} \geq_{s} L^{-}$for all $i$. Hence $L^{-}=L$ and $\eta_{s}\left(F_{i}\right) \rightarrow \eta_{s}(F)$. 

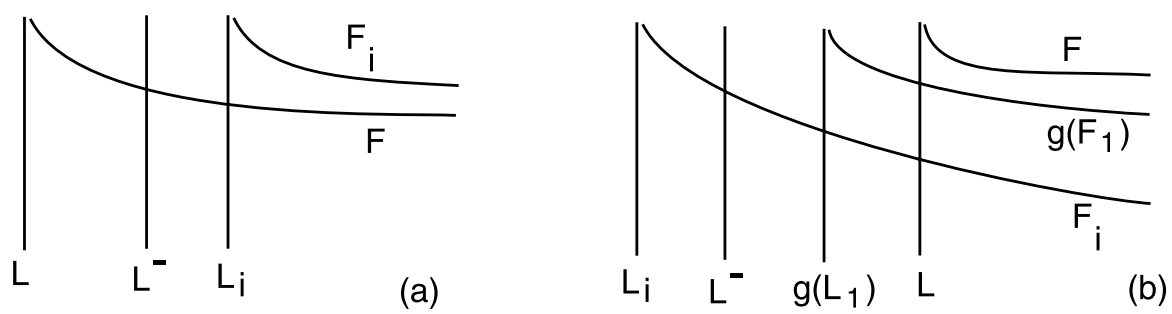

Figure 17. (a) The case $F_{i}$ is decreasing. (b) The case $F_{i}$ is increasing.

$\underline{\text { Case } 2}-F_{i}$ is increasing in $\mathcal{H}$.

Suppose that $L_{i}=\eta_{s}\left(F_{i}\right) \rightarrow L^{-}$with $L^{-}<_{s} L=\eta_{s}(F)$; see Figure 17(b). Then choose a covering translate $g\left(L_{1}\right)$ with

$$
L^{-} \quad<_{s} \quad g\left(L_{1}\right) \quad<_{s} \quad L .
$$

The map $\eta_{s}$ is weakly monotonic, hence $g\left(F_{1}\right)<F$ in $\mathcal{H}$. But also as $\eta_{s}\left(g\left(F_{1}\right)\right)=$ $g\left(L_{1}\right)>_{s} L^{-}$, then $g\left(F_{1}\right)>F_{i}$ in $\mathcal{H}$ for any $i$. This contradicts the fact that $F_{i} \rightarrow F$; see Figure 17(b). This shows that $L^{-}=L$ and so $\eta_{s}\left(F_{i}\right) \rightarrow \eta_{s}(F)$.

It now follows that $\eta_{s}$ is continuous as desired.

Corollary 7.4. For any stable leaf $L \in \widetilde{\mathcal{F}}^{\text {s }}$ there is $F \in \widetilde{\mathcal{F}}$ with $L=\eta_{s}(F)$. In addition $\eta_{s}^{-1}(L)$ is a compact interval in $\mathcal{H}$.

Proof. To prove the first statement choose $g_{0}, g_{1} \in \pi_{1}(M)$ with $g_{0}\left(L^{*}\right)<_{s} L<_{s}$ $g_{1}\left(L^{*}\right)$. Then $g_{0}\left(L^{*}\right)=\eta_{s}\left(g_{0}\left(F^{*}\right)\right)$ and $g_{1}\left(L^{*}\right)=\eta_{s}\left(g_{1}\left(F^{*}\right)\right)$ are in the image of $\eta_{s}$. As $\eta_{s}$ is continous, its image is connected, which implies that $L$ is also in the image of $\eta_{s}$. The second statement follows from $\eta_{s}$ being continuous, surjective, monotonic and $\mathcal{H}$ homeomorphic to $\mathbf{R}$.

We now show that leaves of $\widetilde{\mathcal{F}}$ are boundedly near leaves of $\widetilde{\mathcal{F}}^{s}$.

Proposition 7.5. Let $F \in \widetilde{\mathcal{F}}^{s}$ and $L=\eta_{s}(F)$. Then the Hausdorff distance between $F$ and $L$ is finite and bounded by a global constant $r_{0}$.

Proof. Suppose not. Then there are $F_{i} \in \widetilde{\mathcal{F}}, L_{i}=\eta_{s}\left(F_{i}\right)$ which are not a bounded distance from each other.

First option - Up to subsequence there are $q_{i} \in L_{i}$ with $d\left(q_{i}, F_{i}\right) \rightarrow+\infty$.

Up to covering translations we may assume that $q_{i} \rightarrow q_{0}$, so $L_{i} \rightarrow L_{0} \in \widetilde{\mathcal{F}}^{s}-$ the limit is unique because $\mathcal{F}^{s}$ is $\mathbf{R}$-covered. Therefore the $L_{i}$ are in compact interval $I$ of $\mathcal{H}^{s}$. It follows that $F_{i}$ are in $\eta_{s}^{-1}(I) \subset \mathcal{H}$, which is a compact interval of $\mathcal{H}$ by lemma 7.3 and corollary 7.4 Up to taking a subsequence we may assume that $F_{i} \rightarrow F$ in $\mathcal{H}$. Take $z \in F$ and choose $z_{i} \in F_{i}$ with $z_{i} \rightarrow z$. Then

$$
d\left(q_{i}, F_{i}\right) \leq d\left(q_{i}, z_{i}\right) \rightarrow d(q, z) .
$$

So $d\left(q_{i}, F_{i}\right)$ is bounded, contradiction.

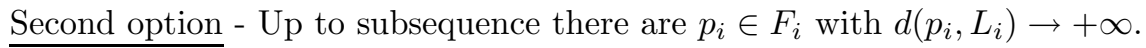

Again up to covering translations, assume that $p_{i} \rightarrow p$ so $F_{i} \rightarrow F$ for some $F \in \mathcal{H}$. By continuity of $\eta_{s}, L_{i}=\eta_{s}\left(F_{i}\right) \rightarrow \eta_{s}(F)=L$. The argument in the first option shows that $d\left(p_{i}, L_{i}\right)$ is bounded, again contradiction to assumption. This finishes the proof of the proposition. 
The goal now is to show that $\mathcal{F}$ is topologically equivalent to $\mathcal{F}^{s}$. However this is not true in general for the following easy reason: given a foliation $\mathcal{F}$ transverse to $\Phi$ one can blow up a leaf (or a collection) of leaves of $\mathcal{F}$ into foliated $I$-bundles Ga-Oe and still get a foliation transverse to $\Phi$. To get the conjugacy, one first needs to blow down these gaps or foliated $I$-bundles. At this point we need to differentiate a bit between the two cases:

Lemma 7.6. Suppose that $\Phi$ is a product $\mathbf{R}$-covered Anosov flow. Then for any $F \in \widetilde{\mathcal{F}}, \partial \Theta(F)=l$, with $l$ a stable leaf in $\mathcal{O}$ (there is no unstable boundary in $\Theta(F))$. If $F, F^{\prime} \in \widetilde{\mathcal{F}}$ satisfy $\eta_{s}(F)=\eta_{s}\left(F^{\prime}\right)$, then $\Theta(F)=\Theta\left(F^{\prime}\right)$.

Proof. Suppose that $\Theta(F)$ has an unstable boundary component $u \in \widetilde{\mathcal{F}}_{\mathcal{O}}^{u}$. But $\Phi$ is product type, so $u \cap l \neq \emptyset$, contradiction to two boundary components of $\Theta(F)$ not intersecting. As seen before there can only one stable boundary component. This shows that $\partial \Theta(F)$ is a single stable leaf $l=\Theta\left(\eta_{s}(F)\right)$. Hence if $\eta_{s}(F)=\eta_{s}\left(F^{\prime}\right)$, then $\partial \Theta(F)=\partial \Theta\left(F^{\prime}\right)$ and both $F, F^{\prime}$ are contained in the front of $\eta_{s}(F)$, so $\Theta(F)=\Theta\left(F^{\prime}\right)$. This finishes the proof.

In the skewed case we have a different situation.

Proposition 7.7. Suppose that $\Phi$ is skewed. Then for any $F \in \widetilde{\mathcal{F}}$, there are unique $L \in \widetilde{\mathcal{F}}^{s}, U \in \widetilde{\mathcal{F}}^{u}$ with $\partial \Theta(F)=\Theta(L) \cup \Theta(U)$. In addition $U$ and L make a perfect fit and $U=\tau_{+}(L)$. In particular given $F, F^{\prime} \in \widetilde{\mathcal{F}}$ with $\eta_{s}(F)=\eta_{s}\left(F^{\prime}\right)$ then $\Theta(F)=\Theta\left(F^{\prime}\right)$.

Proof. Let $F \in \widetilde{\mathcal{F}}$. By lemma $7.2, \partial \Theta(F)$ can have only one stable boundary component, which is $\Theta(L)$ where $L=\eta_{s}(F)$. If $\Theta(F)$ does not have unstable boundary component, then $\Theta(F)$ is a half plane in $\mathcal{O}$ bounded by $\Theta(L)$. Choose $L_{i} \rightarrow+\infty$ in $\mathcal{H}^{s}$, which implies that $\Theta\left(L_{i}\right) \subset \Theta(F)$. Hence $d_{s}\left(L, L_{i}\right) \rightarrow \infty$. Choose $p_{i} \in L_{i} \cap F$. It follows from the distance equation (2) (from the beginning of the section) applied to $p_{i} \in L_{i}$ and $L \in \widetilde{\mathcal{F}}^{s}$, that $d\left(p_{i}, L\right) \rightarrow+\infty$. But $p_{i} \in F$ and proposition 7.5 showed that $d_{H}(F, L)<r_{0}$ so there are $z_{i} \in L$ with $d\left(p_{i}, z_{i}\right)<r_{0}$. This contradicts $d\left(p_{i}, L\right) \rightarrow \infty$.

We conclude that $\Theta(F)$ must have unstable boundary. In addition it can have only one unstable boundary component - the argument is the same as in the proof of proposition 7.1 Let this unstable boundary be denoted by $\eta_{u}(F)$. In the same way as in lemma 7.3. corollary 7.4 and proposition 7.5. it follows that $\eta_{u}$ is continuous, weakly monotone increasing, surjective and for any $F \in \widetilde{\mathcal{F}}$, then $F$ and $\eta_{u}(F)$ are a bounded Hausdorff distance from each other. We stress that $\eta_{u}$ is only defined in the skewed case (given the assumption that there is $F^{*} \in \widetilde{\mathcal{F}}$ with $\Theta(F)$ having stable boundary).

Given $L \in \widetilde{\mathcal{F}}^{s}$ let $J=\left[F_{0}, F_{1}\right]=\eta_{s}^{-1}(L)$ be a non-empty, compact interval in $\mathcal{H}$. Assume $F_{0}<F_{1}$ in $\mathcal{H}$. Given $g \in \pi_{1}(M)$, suppose that $g(J) \cap J \neq \emptyset$. Then some leaf $F^{\prime} \in g(J)$ has $\eta_{s}\left(F^{\prime}\right)=L$. But all leaves in $g(J)$ have the same image under $\eta_{s}$. It follows that $g(J) \subset J$; in the same way $J \subset g(J)$. So the translates of $J$ are either equal to $J$ or disjoint from it. It follows that the stabilizers of $L, F_{0}$ and $F_{1}$ in $\pi_{1}(M)$ are exactly the same. Notice that this also works in the product case.

We now prove that if $\eta_{s}(F)=L$, then $\eta_{u}(F)=\tau_{+}(L)$.

We first assume that $L$ is periodic, that is, there is a non-trivial $g \in \pi_{1}(M)$ with $g(L)=L$. Let $J=\left[F_{0}, F_{1}\right]=\eta_{s}^{-1}(L)$. Then $g\left(F_{0}\right)=F_{0}$. Let $U=\tau_{+}(L)$, which 

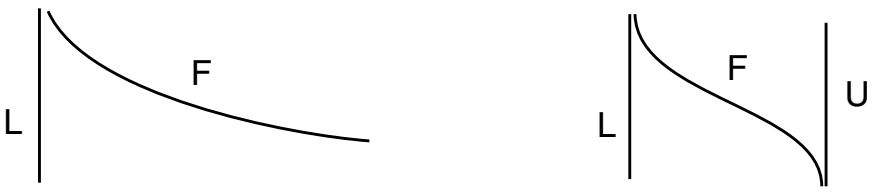

Figure 18. (a) Boundary in the product case. (b) Boundary in the skewed case.

makes a perfect fit with $L$ and is contained in the same component of $\widetilde{M}-L$ which contains $F_{0}$. Then $g(U)=U$. Let $\gamma$ be the periodic orbit of $\widetilde{\Phi}$ in $U$ so $g(\gamma)=\gamma$. If $F_{0} \cap U \neq \emptyset$, then $F_{0}$ intersects all orbits in $U$ - because the only stable boundary to $\Theta\left(F_{0}\right)$ is $\Theta(L)$. Hence $F_{0}$ intersects $\gamma$. Recall that the intersection of $F_{0}$ with $\gamma$ can be at most one point and let this be $p$. Then

$$
g(p)=g\left(F_{0} \cap \gamma\right)=g\left(F_{0}\right) \cap g(\gamma)=F_{0} \cap \gamma=p,
$$

which obviously contradict the fact that $g$ acts freely in $\widetilde{M}$. It follows that $F_{0} \cap U=$ $\emptyset$. On the other hand,

$$
g\left(\eta_{u}\left(F_{0}\right)\right)=\eta_{u}\left(g\left(F_{0}\right)\right)=\eta_{u}\left(F_{0}\right)
$$

But $U$ is the smallest (in $\widetilde{\mathcal{F}}^{u}$ ) leaf contained in $\widetilde{M}-L$ and intersecting stable leaves $>_{s} L$, which is invariant by $g$. Hence either $U=\eta_{u}\left(F_{0}\right)$ or $U$ separates $\eta_{u}\left(F_{0}\right)$ from $L$. The second option would imply $F_{0} \cap U \neq \emptyset$, a contradiction. We conclude that $U=\eta_{u}\left(F_{0}\right)$ and so $\eta_{u}\left(F_{0}\right)=\tau_{+}(L)$. In the same way one proves that $\eta_{u}\left(F_{1}\right)=U$ and so for any $F \in\left[F_{0}, F_{1}\right]$, then $\eta_{u}(F)=\eta_{+}(L)$.

If $L$ is now any leaf in $\widetilde{\mathcal{F}}^{s}$, choose $L_{i} \in \widetilde{\mathcal{F}}^{s}$ periodic with $L_{i} \rightarrow L$. Choose $F_{i} \in \eta_{s}^{-1}\left(L_{i}\right)$. Then as seen before, we may assume up to subsequence that $F_{i} \rightarrow F$. As proved in the previous paragraph, $\eta_{u}\left(F_{i}\right)=U_{i}=\tau_{+}\left(L_{i}\right)$. As $\tau_{+}$is continuous, then $U_{i} \rightarrow U=\tau_{+}(L)$. But $\eta_{u}$ is also continuous, hence $\eta_{u}\left(F_{i}\right)=U_{i} \rightarrow U=\eta_{u}(F)$. It follows that $\eta_{u}(F)=\tau_{+}(L)=\tau_{+}\left(\eta_{s}(F)\right)$ for an arbitrary $F \in \widetilde{\mathcal{F}}$.

Finally this shows that given any $F, F^{\prime} \in \widetilde{\mathcal{F}}$, if $\eta_{s}(F)=\eta_{s}\left(F^{\prime}\right)$, then $\eta_{u}(F)=$ $\eta_{u}\left(F^{\prime}\right)$ also. It follows that $\Theta(F)=\Theta\left(F^{\prime}\right)$, that is, $F$ and $F^{\prime}$ intersect exactly the same orbits of $\widetilde{\Phi}$. This finishes the proof of the proposition.

Given any foliation $\mathcal{F}^{*}$ transverse to $\Phi$ and $F \in \widetilde{\mathcal{F}}^{*}$, let $\partial^{s} \Theta(F)$ be the union of the stable boundary components of $\Theta(F)$ and similarly define $\partial^{u} \Theta(F)$. In our situation of $\mathcal{F}$ transverse to $\Phi, \partial^{s} \Theta(F)=\Theta\left(\eta_{s}(F)\right), \quad \partial^{u} \Theta(F)=\Theta\left(\eta_{u}(F)\right)$ (the second term only occurs in the skewed case).

Proposition 7.8. There is a foliation $\mathcal{F}^{\prime}$, which is obtained from $\mathcal{F}$ by blowing down at most countably many foliated I-bundles of $\mathcal{F}$ to single leaves and so that $\mathcal{F}^{\prime}$ satisfies: $\mathcal{F}^{\prime}$ is transverse to $\Phi$ and for any $L \in \widetilde{\mathcal{F}}^{\text {s }}$ there is a unique $F \in \widetilde{\mathcal{F}}^{\prime}$ with $\Theta(L)=\partial^{s} \Theta(F)$.

Proof. Suppose there is $L \in \widetilde{\mathcal{F}}^{s}$ and there are $F, F^{\prime} \in \widetilde{\mathcal{F}}$ with $\Theta(L)=\partial^{s} \Theta(F)=$ $\eta_{s}(F)=\partial^{s} \Theta\left(F^{\prime}\right)=\eta_{s}\left(F^{\prime}\right)$. Let $J=\eta_{s}^{-1}(L)=\left[F_{0}, F_{1}\right]$, which is a compact, nondegenerate interval in $\mathcal{H}^{s}$. Assume $F_{0}<_{s} F_{1}$. By proposition 7.5, the Hausdorff distances from $L$ to $F_{0}$ and from $L$ to $F_{1}$ are bounded by $r_{0}$. It follows that the Hausdorff distance from $F_{0}$ to $F_{1}$ is bounded by $2 r_{0}$. 
By lemma 7.6 and proposition 7.7, then for any $F, F^{\prime} \in J, \Theta(F)=\Theta\left(F^{\prime}\right)$, that is, $F$ and $F^{\prime}$ intersect exactly the same orbits of $\widetilde{\Phi}$. There is a homeomorphism from $F_{0}$ to $F_{1}$ obtained by following orbits of $\widetilde{\Phi}$. Also for any $g \in \pi_{1}(M), g(J)=J$ or $g(J) \cap J=\emptyset$, so the stabilizers of $F_{0}$ and $F_{1}$ are equal to a subgroup $\mathbf{A}$ of $\pi_{1}(M)$. Let $V$ be the closed subset of $\widetilde{M}$ bounded by $F_{0} \cup F_{1}$. The conditions above imply that $V / \mathbf{A}$ embeds in $M$ and is homeomorphic to $\pi\left(F_{0}\right) \times[0,1]$ with $\pi\left(F_{0}\right)$ and $\pi\left(F_{1}\right)$ corresponding to levels 0 and 1 respectively and following orbit segments of $\Phi$ produces a homeomorphism from $\pi\left(F_{0}\right)$ to $\pi\left(F_{1}\right)$. Therefore $V / \mathbf{A}$ is a foliated product so that transverse segments are given by orbits of $\Phi$. Collapse $\pi\left(F_{0}\right)$ to $\pi\left(F_{1}\right)$ by identifying those segments to points. This is the inverse operation of blowing up a single leaf. There are at most countably many such regions, corresponding exactly to the non-degenerate intervals of $\mathcal{H}$, where $\eta_{s}$ is constant. Do the blow-down operation for the at most countable foliated products as above to produce a foliation $\mathcal{F}^{\prime}$. Then $\mathcal{F}^{\prime}$ is still transverse to the flow $\Phi$. In addition for any $F, F^{\prime} \in \widetilde{\mathcal{F}}^{\prime}$, if $\partial^{s} \Theta(F)=\partial^{s} \Theta\left(F^{\prime}\right)$, then $F=F^{\prime}$ so $\mathcal{F}^{\prime}$ satisfies the requirements of the proposition.

The goal now is to show that $\mathcal{F}^{\prime}$ is topologically conjugate to $\mathcal{F}^{s}$. First we need a couple of facts. Given a leaf $F$ of a foliation $\mathcal{F}$ let $d_{F}$ be the path metric in $F$ $-d_{F}(x, y)$ is the infimum of the lengths of paths from $x$ to $y$ contained in $F$. The metric $d$ will always denote the original metric in $M$ or $\widetilde{M}$.

Lemma $7.9([\mathrm{Fe} 2])$. Let $\mathcal{F}$ be an $\mathbf{R}$-covered foliation in $M^{3}$ closed. Then for any $u>0$ there is $v(u)>0$ satisfying: for any leaf $G$ of $\widetilde{\mathcal{F}}$ and any two points $x, y \in G$ :

$$
d(x, y)<u \Rightarrow d_{G}(x, y)<v(u) .
$$

The point here is that $v(u)$ depends only on $\mathcal{F}$ and $u$ and not on $G, x$ or $y$.

Definition 7.10. Let $\varphi:\left(M_{1}, d_{1}\right) \rightarrow\left(M_{2}, d_{2}\right)$ be a map between metric spaces. Then $\varphi$ is a quasi-isometric embedding [Th2, Gr] if there is $k>0$ so that for any $x, y \in M_{1}$ then

$$
\frac{1}{k} d_{1}(x, y)-k<d_{2}(\varphi(x), \varphi(y))-k<k d_{1}(x, y)+k .
$$

If in addition there is $k^{\prime}$ so that

$$
\forall z \in M_{2}, \quad \exists x \in M_{1}, \quad \text { with } \quad d_{2}(z, \varphi(x))<k^{\prime},
$$

then $\varphi$ is called a quasi-isometry between $M_{1}$ and $M_{2}$. If the constant is important, then we say that $\varphi$ is a $k$ quasi-isometry.

In the case of quasi-isometry it easily follows that there is $\varphi^{\prime}: M_{2} \rightarrow M_{1}$ which is also a quasi-isometry and which is an inverse of $\varphi$ in the quasi-isometry category: $\varphi^{\prime} \circ \varphi$ is a bounded distance from the identity map of $M_{1}$ and similarly for $\varphi \circ \varphi^{\prime}$. Notice that it is NOT at all required that the quasi-isometry $\varphi$ is continuous one is only interested in what happens for large distances. For background on quasi-isometries we refer the reader to $\mathrm{Gr}$, Gh-Ha, $\mathrm{CDP}$.

Let $\mathcal{H}^{\prime}$ be the leaf space of $\widetilde{\mathcal{F}}^{\prime}$ which is obviously homeomorphic to $\mathbf{R}$. Notice that $\mathcal{F}^{\prime}$ is transverse to $\Phi$. Clearly for any $F^{\prime} \in \widetilde{\mathcal{F}}^{\prime}$ there is $F \in \widetilde{\mathcal{F}}$ with $\Theta(F)=\Theta\left(F^{\prime}\right)$. Hence $\partial^{s}\left(F^{\prime}\right)=\partial^{s}(F)=\eta_{s}(F)$. Define $\eta\left(F^{\prime}\right)=\eta_{s}(F)$. By proposition [7.8, the map $\eta$ is well defined and $\eta$ is a homeomorphism from $\mathcal{H}^{\prime}$ to $\mathcal{H}^{s}$. 
Since $\mathcal{F}^{\prime}$ is obtained from $\mathcal{F}$ by collapsing at most countably many foliated products, there is a collapsing map $\beta: M \rightarrow M$ sending leaves of $\mathcal{F}$ to leaves of $\mathcal{F}^{\prime}$ and collapsing the required foliated products. The map $\beta$ is continuous and is a homotopy equivalence which is homotopic to the identity. If lifts to a map $\widetilde{\beta}: \widetilde{M} \rightarrow \widetilde{M}$, which leaves orbits of $\widetilde{\Phi}$ invariant and moves points of $\widetilde{M}$ a bounded distance. Hence the Hausdorff distance $d_{H}(\widetilde{\beta}(F), F)$ is finite and bounded for any $F \in \widetilde{\mathcal{F}}$. Let $F^{\prime}=\widetilde{\beta}(F)$. Since $d_{H}\left(F, \eta_{s}(F)\right)<r_{0}$ and $\eta\left(F^{\prime}\right)=\eta_{s}(F)$, it follows that $d_{H}\left(F^{\prime}, \eta\left(F^{\prime}\right)\right)$ is globally bounded by a constant $r_{1}$ for any $F^{\prime} \in \mathcal{F}^{\prime}$.

We begin by relating leaves of $\widetilde{\mathcal{F}}^{\prime}$ and $\widetilde{\mathcal{F}}^{s}$ metrically.

Lemma 7.11. For any $F \in \widetilde{\mathcal{F}}^{\prime}$ and $\eta(F) \in \widetilde{\mathcal{F}}^{\text {s }}$ there is a roughly natural map $\varphi: \eta(F) \rightarrow F$ which is a uniform quasi-isometry.

Proof. The term roughly natural will become clear in the proof. Both $\mathcal{F}^{s}$ and $\mathcal{F}^{\prime}$ are $\mathbf{R}$-covered. Let $v$ be the function given by lemma 7.9 for $\mathcal{F}^{s}$ and $v^{\prime}$ the respective function for $\mathcal{F}^{\prime}$. Given $F \in \widetilde{\mathcal{F}}^{\prime}$ let $L=\eta(F)$. Then $d_{H}(F, L)<r_{1}$. So given $p \in L$ choose a point $q \in F$ with $d(p, q)<r_{1}$. Let this be $\varphi(p)$. Obviously $\varphi(p)$ is not uniquely defined, but we show it is roughly uniquely defined in $F$ as follows: if $q^{\prime} \in F$ with $d\left(q^{\prime}, p\right)<r_{1}$, then $d\left(q, q^{\prime}\right)<2 r_{1}$. Let $a_{0}=v^{\prime}\left(2 r_{1}\right)$. By lemma $7.9 d_{F}\left(q, q^{\prime}\right)<v^{\prime}\left(2 r_{1}\right)=a_{0}$, which is a fixed constant. So the diameter of the set of possible choices for $\varphi(p)$ is globally bounded and $\varphi(p)$ is well defined in $F$ up to a distance $a_{0}$. Notice that clearly there is no guarantee that $\varphi$ is even continuous.

Given $x, y \in L$ let $n=\left\lfloor d_{L}(x, y)\right\rfloor$ where \lfloor\rfloor denotes the function greatest integer less than or equal the given number. Choose a minimal length geodesic $\gamma$ in $L$ from $x$ to $y$ and choose points $p_{0}, p_{1}, \ldots, p_{n+1}$ in $\gamma$ so that $p_{0}=x, p_{n+1}=y$, $d_{L}\left(p_{i}, p_{i+1}\right)=1$ if $0 \leq i<n$ and $d_{L}\left(p_{n}, p_{n+1}\right)<1$. Let $q_{i}=\varphi\left(p_{i}\right)$. Then $d\left(q_{i}, p_{i}\right)<r_{1}$, hence $d\left(q_{i}, q_{i+1}\right)<2 r_{1}+1$. Let $a_{1}=v^{\prime}\left(2 r_{1}+1\right)$. By lemma 7.9 $d_{F}\left(q_{i}, q_{i+1}\right)<a_{1}$. Therefore

(6) $d_{F}(\varphi(x), \varphi(y))=d_{F}\left(q_{0}, q_{i+1}\right)<(n+1) a_{1}=n a_{1}+a_{1} \leq a_{1} d_{L}(x, y)+a_{1}$.

This proves one side of the needed quasi-isometric inequality (4) for the map $\varphi$. To prove the other direction we will use a dual argument.

Given $w \in F$ choose $z \in \eta(F)=L$ with $d(z, w)<r_{1}$. Let this $z$ be $\varphi^{\prime}(w)$. As above the function $\varphi^{\prime}$ is (roughly) natural - its possible values are in a set of diameter $<2 r_{1}$ in $\widetilde{M}$ and hence of diameter $<v\left(2 r_{1}\right)$ in $L$.

Let $a_{2}=v\left(2 r_{1}+1\right)$. An argument entirely analogous as above shows that for any $z, w \in F$ then

$$
d_{L}\left(\varphi^{\prime}(z), \varphi^{\prime}(w)\right) \leq a_{2} d_{F}(z, w)+a_{2}
$$

Notice that for any $z \in F$ then $\varphi^{\prime}(z) \in L$ and $d\left(z, \varphi^{\prime}(z)\right)<r_{1}$. Let $y=\varphi^{\prime}(z)$. Then $\varphi(y) \in F$ and $d(z, \varphi(y))<2 r_{1}$. So $d_{F}(z, \varphi(y))<v^{\prime}\left(2 r_{1}\right)=a_{0}$. Since $z$ is arbitrary in $F$ and $F$ arbitrary in $\mathcal{H}$, this proves the second condition (5) of the quasi-isometry definition for $\varphi$ with $k^{\prime}=a_{0}$. In addition for any $z \in F$, $d_{F}\left(z, \varphi \circ \varphi^{\prime}(z)\right)<v^{\prime}\left(2 r_{1}\right)=a_{0}$. Let $a_{3}=v\left(2 r_{1}\right)$. In a completely analogous fashion, for any $x \in L$ then

$$
d_{L}\left(x, \varphi^{\prime} \circ \varphi(x)\right)<v\left(2 r_{1}\right)=a_{3} .
$$


Finally we show the other side of the required inequality (4). Let $x, y \in L$ be arbitrary. Then (7) applied to $z=\varphi(x), w=\varphi(y)$ shows that

$$
d_{L}\left(\varphi^{\prime} \circ \varphi(x), \varphi^{\prime} \circ \varphi(y)\right) \leq a_{2} d_{F}(\varphi(x), \varphi(y))+a_{2} .
$$

But $d_{L}\left(\varphi^{\prime} \circ \varphi(x), x\right)<a_{3}$ and similarly for $y$, hence

$$
d_{L}(x, y)<a_{2} d_{F}(\varphi(x), \varphi(y))+\left(a_{2}+2 a_{3}\right)
$$

or

$$
\frac{1}{a_{2}} d_{L}(x, y)-\left(1+\frac{2 a_{3}}{a_{2}}\right)<d_{F}(\varphi(x), \varphi(y)) .
$$

Choose $k=\max \left(a_{1}, a_{2}, 1+\frac{2 a_{3}}{a_{2}}\right)$. Then (4) holds for any $x, y \in L$. Therefore $\varphi$ is a $k$ quasi-isometry from $L$ to $F$. This finishes the proof of the lemma.

We now produce a flow $\Phi^{\prime}$ tangent to $\mathcal{F}^{\prime}$ which "shadows" $\Phi$ in a particular way: for each orbit of $\widetilde{\Phi}$ there is an orbit of $\widetilde{\Phi}^{\prime}$ which is a bounded Hausdorff distance from it. Recall that a quasigeodesic in a metric space is a quasi-isometric embedding of $\mathbf{R}$ or $\mathbf{Z}$ (with their natural metrics).

Proposition 7.12 (shadowing flow). There is a flow $\Phi^{\prime}$ in $M$ tangent to $\mathcal{F}^{\prime}$ which shadows $\Phi$ : for any orbit $\gamma$ of $\widetilde{\Phi}$ there is unique orbit $\gamma^{\prime}$ of $\widetilde{\Phi}^{\prime}$ associated to it and $\gamma^{\prime}$ is is a bounded distance from $\gamma$.

Proof. It is well known that the leaves of $\widetilde{\mathcal{F}}^{s}$ are Gromov negatively curved in the large in their path metrics [Su, Pl1,, $\mathrm{Gr}$. Therefore each leaf $L$ of $\widetilde{\mathcal{F}}^{s}$ has an ideal boundary $\partial_{\infty} L$ which is then homeomorphic to a circle Fe2] and $L \cup \partial_{\infty} L$ is homeomorphic to a closed disk [Fe2]. Given $F \in \widetilde{\mathcal{F}}^{\prime}$ then $F$ is $k$ quasi-isometric to $\eta(F)=L$ - it follows that $F$ is also Gromov negatively curved in the large Gr, Gh-Ha, CDP.

Since all leaves of $\widetilde{\mathcal{F}}^{\prime}$ are Gromov negatively curved, Candel Can showed that there is a metric $d^{\prime}$ in $M$ making leaves of $\mathcal{F}^{\prime}$ hyperbolic. The metric $d^{\prime}$ is obviously smooth along the leaves of $\mathcal{F}^{\prime}$ but may be only $C^{0}$ transversely. Here we use both metrics $d$ and $d^{\prime}$. Let $d_{F}^{\prime}$ be the induced path metric in each leaf of $\mathcal{F}^{\prime}-$ it is a hyperbolic metric. Then the metrics $d_{F}$ and $d_{F}^{\prime}$ are uniformly quasi-isometric, that is, the identity map $\left(F, d_{F}\right) \rightarrow\left(F, d_{F}^{\prime}\right)$ is a uniform quasi-isometry. Uniform means that the quasi-isometric constant does not depend on $F$ and this follows from compactness of $M$.

Let $L=\eta(F)$. The flow lines of $\widetilde{\Phi}$ in $L$ are uniform quasigeodesics in $L$ Fe3] and therefore limit to two distinct ideal points in $\partial_{\infty} L$ [Fe3]. In fact there is a common forward ideal point $b(L)$ of all flow lines (they are forward asymptotic), and all other points of $\partial_{\infty} L$ are negative ideal points of flow lines of $\widetilde{\Phi}$ in $L[\mathrm{Fe} 3$.

An important fact about Gromov negatively curved spaces is that since $\varphi: L \rightarrow$ $F$ is a quasi-isometry, it extends to a homeomorphism $\bar{\varphi}: L \cup \partial_{\infty} L \rightarrow F \cup \partial_{\infty} F$ Gr, Gh-Ha, CDP. This defines a point $b(F) \in \partial_{\infty} F$ which is the image of $b(L)$ under this map. The point $b(F)$ is uniquely defined in $\partial_{\infty} F$. We now construct a flow $\widetilde{\Phi}^{\prime}$ in $\widetilde{M}$ as follows: its orbits are contained in leaves of $\widetilde{\mathcal{F}}^{\prime}$. For each $F \in \widetilde{\mathcal{F}}^{\prime}$ the orbits are geodesics in the hyperbolic metric $d_{F}^{\prime}$ of $F$. They all have one ideal point in $b(F)$ and move toward this ideal point with speed 1.

We first need to show that this defines a continuous flow in $\widetilde{M}$. In each leaf of $\widetilde{\mathcal{F}}^{\prime}$ it is obviously smooth but we need to consider what happens transversally. Given 


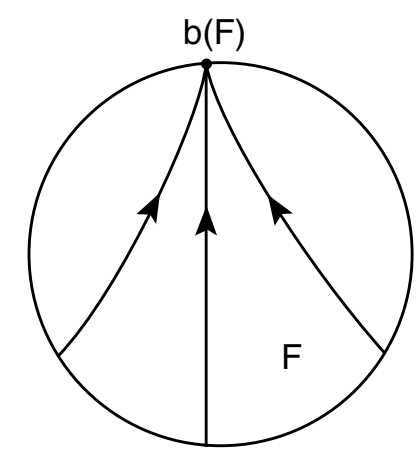

Figure 19. The shadowed flow in $\widetilde{\mathcal{F}}^{\prime}$.

$p \in \widetilde{M}$ let $\mathrm{D} \widetilde{\Phi}^{\prime}(p)$ be the tangent vector field to the flow lines. We aim to prove that $\mathrm{D} \widetilde{\Phi}^{\prime}$ is continuous with $p$.

Let $\mathcal{O}^{\prime}$ be the orbit space of $\widetilde{\Phi}^{\prime}$. Before proving continuity we produce a map from orbits of $\widetilde{\Phi}$ to orbits of $\widetilde{\Phi}^{\prime}$ or equivalently a map from $\mathcal{O}$ to $\mathcal{O}^{\prime}$. Here we identify orbits of $\widetilde{\Phi}$ to points of $\mathcal{O}$ and similarly for $\mathcal{O}^{\prime}$. Given $\gamma$ and orbit of $\widetilde{\Phi}$, it is contained in $L \in \widetilde{\mathcal{F}}^{s}$. Let $F=\eta^{-1}(L) \in \widetilde{\mathcal{F}}^{\prime}$. Notice that $F$ is unique. Then $\gamma$ is a uniform quasigeodesic in $L[\mathrm{Fe}]$ and as $\varphi: L \rightarrow F$ is a quasi-isometry then $\varphi(\gamma)$ is a quasigeodesic in $F$. Since $\varphi$ is not continuous, $\varphi(\gamma)$ may not be a continuous curve. Another point of view is to choose a sequence of points in $\gamma$ so that consecutive points are 1 unit apart in $\gamma$. The image is a quasi-isometric embedding from $\mathbf{Z}$ in $F$ and its image is a bounded distance from a unique geodesic in $F$ Gr, Gh-Ha, CDP. One of the ideal points is obviously $b(F)$ and the other depends on $\gamma$. There is a unique geodesic in $F$ which has these two ideal points and let this be $\chi(\gamma)$. The geodesic $\chi(\gamma)$ is an orbit of $\widetilde{\Phi}^{\prime}$ and this defines a map $\chi: \mathcal{O} \rightarrow \mathcal{O}^{\prime}$. Then $\chi(\gamma)$ is a bounded Hausdorff distance from $\varphi(\gamma)$ and hence from $\gamma$ also. In fact $\chi(\gamma)$ is the unique geodesic in $F$ which is bounded distance from $\gamma$ in $F$ and hence in $\widetilde{M}$. The bound is global for all orbits of $\widetilde{\Phi}$. The map $\chi$ sends the set of orbits of $\widetilde{\Phi}$ in $L$ bijectively to the set of orbits of $\widetilde{\Phi}^{\prime}$ in $F$. Finally $\chi: \mathcal{O} \rightarrow \mathcal{O}^{\prime}$ is a bijection.

We now prove continuity of $\mathrm{D} \Phi^{\prime}$. Let $p_{i} \rightarrow p$ in $\widetilde{M}$. Let $\alpha_{i}$ be the orbits of $\widetilde{\Phi}^{\prime}$ through $p_{i}$. We can choose a subsequence so that $\mathrm{D} \Phi^{\prime}\left(p_{i}\right)$ converges to a tangent vector $w_{0}$ at $p$. Let $p_{i} \in F_{i} \in \widetilde{\mathcal{F}}^{\prime}$ and $p \in F \in \widetilde{\mathcal{F}}^{\prime}$. Let $\alpha$ be the geodesic of $F$ through $p$ in the direction of $w_{0}$. Then every point of $\alpha$ is a limit of points in $\alpha_{i}$. Let $L_{i}=\eta\left(F_{i}\right)$ and $\gamma_{i} \subset L_{i}$ orbits of $\widetilde{\Phi}$ which are a uniform bounded distance from the orbits $\alpha_{i}$. By the construction above the $\gamma_{i}$ are unique. Also $L_{i} \rightarrow L$ with $L=\eta(F)$. The $\gamma_{i}$ all have points $q_{i}$ a bounded distance from $p_{i}$ so up to a further subsequence we may assume that $q_{i} \rightarrow q$ and $\gamma_{i}$ converge to an orbit $\gamma$ of $\widetilde{\Phi}$ in $L$. For any fixed size $r_{2}$, the segments of size $r_{2}$ of $\gamma_{i}$ centered at $q_{i}$ converge uniformly to the segment of size $r_{2}$ of $\gamma$. But these segments are a bounded distance from segments of size $z\left(r_{2}\right)$ of $\alpha_{i}$ centered at $p_{i}$, where $z\left(r_{2}\right) \rightarrow \infty$ when $r_{2} \rightarrow \infty$. But segments of fixed size in $\alpha_{i}$ converge uniformly to segments in $\alpha$, so for any fixed size segment in $\alpha$ it is a bounded distance from a segment in $\gamma$. In addition the 
bound is independent of the segment or its size. It follows that the whole orbit $\alpha$ is a bounded distance from $\gamma$. But the only geodesic of $F$ which is a bounded distance from the orbit $\gamma$ of $\widetilde{\Phi}$ is $\chi(\gamma)$. Hence $\alpha=\chi(\gamma)$ and the tangent to $\alpha$ at $p$ is $\widetilde{D}^{\prime}(p)$ which is $w_{0}$. This proves continuity of $\mathrm{D} \widetilde{\Phi}^{\prime}$ and shows that $\widetilde{\Phi}^{\prime}$ is a continuous flow in $\widetilde{M}$.

Furthermore the function $b(F)$ is clearly equivariant under covering translations: if $g \in \pi_{1}(M)$ and $F \in \widetilde{\mathcal{F}}^{\prime}$, then $b(g(F))=g(b(F))$ - where the second $g$ denotes the extension of the isometry $g: F \rightarrow g(F)$ to the ideal boundaries of $F$ and $g(F)$. It follows that $\widetilde{\Phi}^{\prime}$ induces a flow $\Phi^{\prime}$ in $M$. Notice that $\chi$ is a homeomorphism from $\mathcal{O}$ to $\mathcal{O}^{\prime}$

Finally for any $F \in \widetilde{\mathcal{F}}^{\prime}, \eta(F)=L \in \widetilde{\mathcal{F}}^{s}$, the maps $\partial_{\infty} L \rightarrow \partial_{\infty} F$ are also equivariant, hence the map $\chi: \mathcal{O} \rightarrow \mathcal{O}^{\prime}$ is also group equivariant.

We now prove topological equivalence of $\mathcal{F}^{\prime}$ and $\mathcal{F}^{s}$.

Theorem 7.13. The flows $\Phi$ and $\Phi^{\prime}$ are topologically conjugate. The foliation $\mathcal{F}^{\prime}$ is topologically equivalent to the stable foliation $\mathcal{F}^{s}$ of $\Phi$.

Proof. By the previous proposition there is a homeomorphism $\chi: \mathcal{O} \rightarrow \mathcal{O}^{\prime}$. This homeomorphism is group equivariant. There is a general theory which deals with such situations Hae: the theory of classifying spaces for flows produces a map $h: M \rightarrow M$ which is a homotopy equivalence and sends orbits of $\Phi$ to orbits of $\Phi^{\prime}$ and is transversely injective. The problem is that $h$ may not be injective along the orbits. Ghys Gh encountered this situation when studying Anosov flows in Seifert fibered spaces and did an averaging trick to produce an injective map, hence topological equivalence. Barbot did the same for general R-covered Anosov flows Ba2. We cannot apply these results directly because we do not know that the flow $\Phi^{\prime}$ is Anosov, let alone that it is a smooth flow. On the other hand there is much more structure in our situation which allows us to get the topological equivalence more directly.

In our situation the homeomorphism $\chi: \mathcal{O} \rightarrow \mathcal{O}^{\prime}$ and the quasi-isometries $\varphi$ : $L \rightarrow \eta^{-1}(L)$ give a way to relate the orbits and points in the orbits. Let $\mathcal{U}$ be a very fine, locally finite foliation box cover $\left(\right.$ of $\mathcal{F}^{s}$ ) of $M$. Choose a partition of unity subordinated to this cover. For each element of the cover, lift it to $\widetilde{M}$ and define a map from this set to a foliated box of $\mathcal{F}^{\prime}$ by sending each plaque of $\widetilde{\mathcal{F}}^{s}$ to a plaque of $\widetilde{\mathcal{F}}^{\prime}$, each orbit segment of $\widetilde{\Phi}$ to an orbit segment of $\widetilde{\Phi}^{\prime}$ so that this is a homeomorphism between the two sets and monotonic increasing along orbits. The final requirement is that any point and its image are $<r_{3}$ distant apart, where $r_{3}$ is a globally fixed constant. This is doable because $d_{H}(\gamma, \chi(\gamma))$ is bounded over all orbits of $\widetilde{\Phi}$. Iterate by covering translations of $\widetilde{M}$. Do this for all elements of the foliated box cover of $M$. Given $p \in \widetilde{M}$ with $p \in \gamma$ orbit of $\widetilde{\Phi}$, then the above construction defines finitely many images of $p$ in $\chi(\gamma)$. Each orbit of $\widetilde{\Phi}^{\prime}$ has a well-defined affine structure: use the partition of unity as affine coordinates of the images of $p$ in the orbit $\chi(\gamma)$ of $\widetilde{\Phi}^{\prime}$ to define a map $\widetilde{h}: \widetilde{M} \rightarrow \widetilde{M}$ which sends orbits of $\widetilde{\Phi}$ to orbits of $\widetilde{\Phi}^{\prime}$ and induces $\chi: \mathcal{O} \rightarrow \mathcal{O}^{\prime}$. This map is continuous and equivariant, producing a map $h: M \rightarrow M$ which is a homotopy equivalence, sends orbits of $\Phi$ to orbits of $\Phi^{\prime}$ and is transversely injective. 
Furthermore the construction implies that for any $p \in \widetilde{M}$, then $d(p, \widetilde{h}(p))<r_{4}$ for a globally fixed constant $r_{4}$. Therefore even though $\widetilde{h}$ may not be monotonic in a fixed orbit of $\widetilde{\Phi}$, it eventually progresses in the forward direction. This is called quasi-monotonic by Thurston [Th5] and it is the key property which allows for the construction of a monotonic map [Ba2] (see details of the proof of theorem 3.4 of Ba2 ). The new map is obtained by averaging $h$ on orbit segments of long but fixed size $t$. This provides a new map $h^{\prime}: M \rightarrow M$, which is now injective along orbits and is therefore a topological equivalence from $\Phi$ to $\Phi^{\prime}$.

This immediately shows that $\mathcal{F}^{\prime}$ is topologically equivalent to $\mathcal{F}^{s}$, finishing the proof of the theorem.

We collect these results together to obtain theorem $\mathrm{C}$ of the introduction - the rigidity theorem:

Corollary 7.14 (Theorem C). Let $\Phi$ be an $\mathbf{R}$-covered pseudo-Anosov flow transverse to a foliation $\mathcal{F}$ and suppose that $\Phi$ is not regulating for $\mathcal{F}$. Then $\mathcal{F}$ is topologically equivalent to a blow up of either (1) the stable foliation $\mathcal{F}^{s}$ or (2) the unstable foliation $\mathcal{F}^{u}$ of $\Phi$.

Proof. Suppose first that some leaf $F \in \widetilde{\mathcal{F}}$ has $\partial^{s}(\Theta(F)) \neq \emptyset$. Then the results of this section show that conclusion (1) occurs. If on the other hand $\partial^{u}(\Theta(F)) \neq \emptyset$, the same arguments show that (2) occurs. This finishes the proof. Notice that in the skewed case $\mathcal{F}^{s}$ and $\mathcal{F}^{u}$ are topologically equivalent Ba2].

Finally we also mention a topological rigidity for the flows: Let $\mathcal{F}$ be an $\mathbf{R}$ covered foliation in $M^{3}$ closed. Then, up to topological conjugacy, there is at most one pseudo-Anosov flow $\Phi$ which is transverse to $\mathcal{F}$, but not regulating. The proof is very similar to the arguments given in this section and is omitted for brevity.

\section{REFERENCES}

[An] D. V. Anosov, Geodesic flows on closed Riemannian manifolds with negative curvature, Proc. Steklov Inst. Math. 90 (1969). MR0242194 (39:3527)

[An-Si] D. V. Anosov and Y. Sinai, Some smoothly ergodic systems, Russian Math. Surveys 22 (1967) 5 103-167. MR0224771 (37:370)

[Ba1] T. Barbot, Géométrie transverse des flots, Thesis, École Normale Sup. Lyon, 1992.

[Ba2] T. Barbot, Caractérization des flots d'Anosov en dimension 3 par leurs feuilletages faibles, Ergod. Th. Dynam. Sys. 15 (1995) 247-270. MR.1332403 (96d:58100)

[Ba3] T. Barbot, Flots d'Anosov sur les variétés graphées au sens de Waldhausen, Ann. Inst. Fourier Grenoble 46 (1996) 1451-1517. MR:1427133 (97j:57031)

[Ba4] T. Barbot, Generalizations of Bonatti-Langevin example of Anosov flow and their classification up to topological equivalence, Comm. Anal. Geom. 6 (1998) 749-798. MR1652255 (99m:58142)

[Bl-Ca] S. Bleiler and A. Casson, Automorphisms of surfaces after Nielsen and Thurston, Cambridge Univ. Press, 1988. MR0964685 (89k:57025)

[Bow1] R. Bowen, Periodic orbits for hyperbolic flows, Amer. Jour. Math. 94 (1970) 1-30. MR0298700 (45:7749)

[Bow2] R. Bowen, Symbolic dynamics for hyperbolic flows, Amer. Jour. Math. 95 (1970) 429-459. MR0339281 (49:4041)

[Br] M. Brittenham, Essential laminations in Seifert fibered spaces, Topology 32 (1993) 61-85. MR.1204407(94c:57027)

[Ca1] D. Calegari, R-covered foliations of hyperbolic 3-manifolds, Geom. Topol 3 (1999) 137153. MR 1695533 (2000c:57038) 
[Ca2] D. Calegari, The geometry of R-covered foliations, Geom. Topol. 4 (2000) 457-515. MR 1800151 (2001k:57016)

[Ca3] D. Calegari, Foliations with one sided branching, Geom. Dedicata 96 (2003) 1-53. MR.1956833 (2003m:57037)

[Ca4] D. Calegari, Promoting essential laminations, Part I, preprint, 2001.

[Can] A. Candel, Uniformization of surface laminations, Ann. Sci. Ecole Norm. Sup. (4) 26 (1993) 489-516. MR1235439 (94f:57025)

[CDP] M. Coornaert, T. Delzant and A. Papadopoulos, Géométrie et théorie des groupes, Les groupes hyperboliques de Gromov, Lecture Notes in Math. 1441 Springer (1991). MR.1075994 (92f:57003)

[Ca-Th] J. Cannon and W. Thurston, Group invariant Peano curves, preprint, 1985.

[CLR1] D. Cooper, D. Long and A. Reid, Bundles and finite foliations, Inven. Math. 118 (1994) 255-283. MR.1292113 (96h:57013)

[CLR2] D. Cooper, D. Long and A. Reid, Finite foliations and similarity interval exchange maps, Topology, 36 (1997) 209-227. MR1410472 (97j:57032)

[FLP] A. Fathi, Laudenbach, Poenaru, Travaux de Thurston sur les surfaces, Séminaire Orsay, Astérisque 66-67 Socieété Mathématique de France, 1979. MR0568308 (82m:57003)

[Fe1] S. Fenley, Asymptotic properties of depth one foliations in hyperbolic 3-manifolds, Jour. Diff. Geom., 36 (1992) 269-313. MR1180384 (93k:57030)

$[\mathrm{Fe} 2] \quad$ S. Fenley, Quasi-isometric foliations, Topology 31 (1992) 667-676. MR 1174265 (94a:57044)

[Fe3] S. Fenley, Anosov flows in 3-manifolds, Ann. of Math. 139 (1994) 79-115. MR1259365 (94m:58162)

[Fe4] S. Fenley, Quasigeodesic Anosov flows and homotopic properties of closed orbits, Jour. Diff. Geo. 41 (1995) 479-514. MR1331975 (96f:58118)

[Fe5] S. Fenley, The structure of branching in Anosov flows of 3-manifolds, Comm. Math. Helv. 73 (1998) 259-297. MR.1611703 (99a:58123)

[Fe6] S. Fenley, Continuous extension of Anosov foliations in 3-manifolds with negatively curved fundamental group, Pac. Jour. Math. 186 (1998) 201-216. MR1663798 (99k:58135)

[Fe7] S. Fenley, Limit sets of foliations in hyperbolic 3-manifolds, Topology 37 (1998) 875-894. MR 1607756 (2000e:57029)

[Fe8] S. Fenley, Surfaces transverse to pseudo-Anosov flows and virtual fibers in 3-manifolds, Topology 38 (1999) 823-859. MR.1679801 (2001a:57041)

[Fe9] S. Fenley, Foliations with good geometry, Journal of the A.M.S. 12 (1999) 619-676. MR.1674739 (2000b:57041)

[Fe10] S. Fenley, Foliations and the topology of 3-manifolds: $\mathbf{R}$-covered foliations and transverse pseudo-Anosov flows, Comm. Math. Helv. 77 (2002) 415-490. MR.1933786|(2003g:57044)

[Fe11] S. Fenley, Pseudo-Anosov flows and inconpressible tori, Geom. Dedicata 99 (2003) 61102. MR1998929 (2004f:57035)

[Fe12] S. Fenley, Topological rigidity of pseudo-Anosov flows transverse to foliations, in preparation.

[Fe-Mo] S. Fenley and L. Mosher, Quasigeodesic flows in hyperbolic 3-manifolds, Topology 40 (2001) 503-537. MR1838993 (2002e:57039)

[Fr-Wi] J. Franks and R. Williams, Anomalous Anosov flows, in Global theory of Dyn. Systems, Lecture Notes in Math. 819 Springer (1980). MR0591182 (82e:58078)

[Ga1] D. Gabai, Foliations and the topology of 3-manifolds, J. Diff. Geo. 18 (1983) 445-503. MR.0723813 (86a:57009)

[Ga2] D. Gabai, Foliations and the topology of 3-manifolds III, J. Diff. Geo. 26 (1987) 479-536. MR0910018 (89a:57014b)

[Ga-Oe] D. Gabai and U. Oertel, Essential laminations and 3-manifolds, Ann. of Math. 130 (1989) 41-73. MR 1005607 (90h:57012)

[Gh] E. Ghys, Flots d'Anosov sur les 3-variétés fibrées en cercles, Ergod. Th and Dynam. Sys. 4 (1984) 67-80. MR0758894 (86b:58098)

[Gh-Ha] E. Ghys and P. de la Harpe, editors, Sur les groupes hyperboliques d'apres Mikhael Gromov, Progress in Math. 83 Birkhäuser, (1996). MR.1086648 (92f:53050)

[Go] S. Goodman, Closed leaves in foliations of codimension one, Comm. Math. Helv. 50 (1975) 383-388. MR0423371 (54:11350) 
[Go-Sh] S. Goodman and S. Shields, A condition for the stability of $\mathbf{R}$-covered for foliations of 3-manifolds, Trans. A.M.S. 352 (2000) 4051-4065. MR.1624178 (2000m:57042)

[Gr] M. Gromov, Hyperbolic groups, in Essays on group theory, Springer, 1987, 75-263. MR0919829 (89e:20070)

[Hae] A. Haefliger, Groupöides d'holonomie et classifiants, Asterisque 116 (1984) 70-97. MR0755163 (86c:57026a)

[Han] M. Handel, Global shadowing of pseudo-Anosov homeomorphisms, Ergod. Th. Dynam. Sys. 5 (1985) 373-377. MR0805836 (87e:58172)

[He] J. Hempel, 3-manifolds, Ann. of Math. Studies 86, Princeton University Press, 1976. MR0415619(54:3702)

[Ko] N. Kopell, Commuting diffeomorphisms, Proceedings of Symposia in Pure Mathematics, A.M.S. 14 (1970) 165-184. MR0270396 (42:5285)

[Le] G. Levitt, Feuilletages des variétés de dimension trois qui son fibrés en cercles, Comm. Math. Helv. 53 (1978) 572-594. MR0511848 (80c:57017)

[Man] B. Mangum, Incompressible surfaces and pseudo-Anosov flows, Topol. Appl. 87 (1998) 29-51. MR.1626072 (99e:57024)

[Mo1] L. Mosher, Dynamical systems and the homology norm of 3-manifolds, I, Duke Jour. Math. 65 (1992) 449-500. MR1154179 (93g:57018a)

[Mo2] L. Mosher, Laminations and flows transverse to finite depth foliations, manuscript available in the web from http://newark.rutgers.edu:80/ mosher/, Part I: Branched surfaces and dynamics, Part II in preparation.

[No] S. P. Novikov, Topology of foliations, Trans. Moscow Math. Soc. 14 (1963) 268-305. MR0200938 (34:824)

[Pl1] J. Plante, Foliations with measure preserving holonomy, Ann. of Math. 107 (1975) 327361. MR0391125 (52:11947)

[P12] J. Plante, Foliations of 3-manifolds with solvable fundamental group, Inven. Math. 51 (1979) 219-230. MR0530629 (80i:57020)

[Pl3] J. Plante, Solvable groups acting on the line, Trans. A.M.S. 278 (1983) 401-414. MR.0697084 (85b:57048)

[Pl4] J. Plante, Anosov flows, transversely affine and a conjecture of Verjovsky, Jour. London Math. Soc. 83 (1981) 359-362. MR0609116 (82g:58069)

[Pl-Th] J. Plante and W. Thurston, Anosov flows and the fundamental group, Topology 11 (1972) 147-150. MR0295389(45:4455)

[Rob1] R. Roberts, Taut foliations in punctured surface bundles, I, Proc. London Math. Soc. 82 (2001) 747-768. MR1816696 (2003a:57040)

[Rob2] R. Roberts, Taut foliations in punctured surface bundles, II, Proc. London Math. Soc. 83 (2001) 443-471. MR1839461(2003j:57016)

[Rol] D. Rolfsen, Knots and links, Publish or Perish, 1976. MR0515288 (58:24236)

[Ros] H. Rosenberg, Foliations by planes, Topology 7 (1968) 131-138. MR0228011 (37:3595)

[Sc] P. Scott, Subgroups of surface groups are almost geometric, Jour. Lond. Math. Soc. 17 (1978) 555-565. MR0494062 (58:12996)

[Se] A. Selberg, On discontinuous groups in higher-dimensional symmetric spaces, in Contributions to Function Theory, Tata Inst. of Fund. Research, Bombay (1960) 147-164. MR 0130324 (24:A188)

[St] K. Strebel, Quadratic differentials, Springer-Verlag, 1984. MR0743423 (86a:30072)

[Su] D. Sullivan, Cycles for the dynamical study of foliated manifolds and complex manifolds, Invent. Math. 36 (1976) 225-255. MR0433464 (55:6440)

[Th1] W. Thurston, Foliations of 3-manifolds that are circle bundles, Ph.D. thesis, University of California Berkeley, 1972.

[Th2] W. Thurston, The geometry and topology of 3-manifolds, Princeton University Lecture Notes, 1982.

[Th3] W. Thurston, On the geometry and dynamics of diffeomorphisms of surfaces, Bull. A.M.S 19 (1988) 417-431. MR0956596 (89k:57023)

[Th4] W. Thurston, Hyperbolic structures on 3-manifolds II, Surface groups and 3-manifolds that fiber over the circle, preprint.

[Th5] W. Thurston, Three manifolds, foliations and circles I, preprint, 1997.

[Th6] W. Thurston, Three manifolds, foliations and circles II, The transverse asymptotic geometry of foliations, preprint, 1998. 
[Th7] W. Thurston, Private communication.

[Ve] A. Verjovsky, Codimension one Anosov flows, Bol. Soc. Mat. Mex. 19 (1977) 49-77. MR0431281(55:4282)

Department of Mathematics, Florida State University, Tallahassee, Florida 32306 4510 